\title{
Modulation of the H-Bond Basicity of Functional Groups by $\alpha$-Fluorine-Containing Functions, and its Implications for Lipophilicity and Bioisosterism
}

\author{
Yossi Zafrani, ${ }^{* a}$ Galit Parvari, ${ }^{b}$ Dafna Amir, ${ }^{a}$ Lee Ghindes-Azaria, ${ }^{a}$ Shlomi Elias, ${ }^{* a}$ Alexander \\ Pevzner, ${ }^{* c}$ Gil Fridkin, ${ }^{a}$ Anat Berliner, ${ }^{a}$ Eytan Gershonov, ${ }^{a}$ Yoav Eichen, ${ }^{b}$ Sigal Saphier,${ }^{* a}$ and \\ Shahaf Katalan ${ }^{d}$
}

${ }^{\text {aDepartment }}$ of Organic Chemistry Israel Institute for Biological Research Ness-Ziona 74100, Israel ${ }^{b}$ Schulich Faculty of Chemistry Technion, Israel Institute of Technology, Technion City, Haifa 3200008, Israel 'Department of Physical Chemistry Israel Institute for Biological Research Ness-Ziona 74100, Israel dDepartment of Pharmacology Israel Institute for Biological Research Ness-Ziona 74100, Israel *E-mail: yossiz@iibr.gov.il ; shlomie@iibr.gov.il; alexanderp@iibr.gov.il; sigals@iibr.gov.il

\section{Table of Contents}

1. Experimental Section S2

1.1 Determination of $\mathrm{p} K_{\mathrm{HB}}$ values S2

1.1.1 $\mathrm{p} K_{\mathrm{HB}}$ measurements by FT-IR S2

1.1.1.1 Method S2

1.1.1.2 IR spectra of compounds $\mathbf{1 - 5}$ S3

1.1.2 $\mathrm{p} K_{\mathrm{HB}}$ measurements by ${ }^{19} \mathrm{~F}-\mathrm{NMR}$ S16

2. ${ }^{19} \mathrm{~F}$ NMR spectra of $\mathbf{2 b}-\mathbf{F}, \mathbf{2} \mathbf{b}-\mathbf{F} \mathbf{2}$ and $\mathbf{2 b}-\mathbf{F} \mathbf{3}$ in octanol and water phases

$2.6{ }^{19} \mathrm{~F}-\mathrm{NMR}$ spectra for $\log P_{\mathrm{o} / \mathrm{w}}$ determination of $\mathbf{2} \mathbf{b}-\mathbf{F}$

$2.7{ }^{19} \mathrm{~F}-\mathrm{NMR}$ spectra for $\log P_{\mathrm{o} / \mathrm{w}}$ determination of the mixture $\mathbf{2 b - F 2 / 6 / 7}$ 


\section{Experimental section}

\section{$\underline{1.1 \text { Determination of } \mathrm{p} K} \underline{\mathrm{HB}} \underline{\text { values. }}$}

\section{$\underline{1.1 .1} \mathrm{p} K_{\mathrm{HB}} \underline{\text { measurements by FT-IR }}$}

\subsubsection{Method}

The $\mathrm{pK}_{\mathrm{HB}}$ determination of acetophenone is illustrated for example in Table 1 and gives $\mathrm{p} K_{\mathrm{HB}}=$ $1.18 \pm 0.04$ which corresponds to literature data. ${ }^{1}$

Table S1. Five determinations of p-FP -acetophenone complex formation constant in $\mathrm{CCl}_{4}$ at $25^{\circ} \mathrm{C}^{\mathrm{a}}$

\begin{tabular}{|c|c|c|c|c|c|c|}
\hline Runs & 0 & 1 & 2 & 3 & 4 & 5 \\
\hline$C \quad \begin{array}{l}0 \\
a\end{array}$ & 2.46 & 2.39 & 2.3 & 2.46 & 2.34 & 2.37 \\
\hline$C \quad \stackrel{0}{b}$ & 0 & 6.85 & 9.07 & 12.2 & 18.6 & 23.8 \\
\hline Absorbance $A$ (p-FP) & 0.566 & 0.493 & 0.469 & 0.481 & 0.421 & 0.415 \\
\hline$C \quad \mathrm{a}=A / \varepsilon l^{b}$ & & 2.14 & 2.04 & 2.09 & 1.83 & 1.8 \\
\hline$C_{c}=C \quad \begin{array}{ll}0 & -C_{a}\end{array}$ & & 0.25 & 0.26 & 0.37 & 0.51 & 0.57 \\
\hline$C_{b}=C \quad \begin{array}{ll}0 \\
b\end{array}-C_{c}$ & & 6.6 & 8.81 & 11.83 & 18.09 & 23.2 \\
\hline$K_{f} / M^{-1}$ & & 17.5 & 14.6 & 14.9 & 15.4 & 13.5 \\
\hline$p K_{H B}$ & & 1.24 & 1.16 & 1.17 & 1.19 & 1.13 \\
\hline
\end{tabular}

${ }^{a}$ All concentrations presented in $\mathrm{mmol} \mathrm{L}^{-1} \cdot C \underset{a}{0}, C{ }_{b}^{0}, C$ a,$C_{b}$ and $C_{c}$ are respectively the initial and equilibrium concentrations of the acid, base and complex. ${ }^{b}$ According to Beer-Lambert law: $\varepsilon=$ $0.566 /\left(2.46 \times 10^{-3}\right)=230.1 \mathrm{M}^{-1} \mathrm{~cm}^{-1}$. 


\subsubsection{IR spectra of compounds 1-5}

The IR range is presented in the range of $2600-4000 \mathrm{~cm}^{-1}$ for all compounds due to the infrasil absorbance in the range of $400-2600 \mathrm{~cm}^{-1}$.
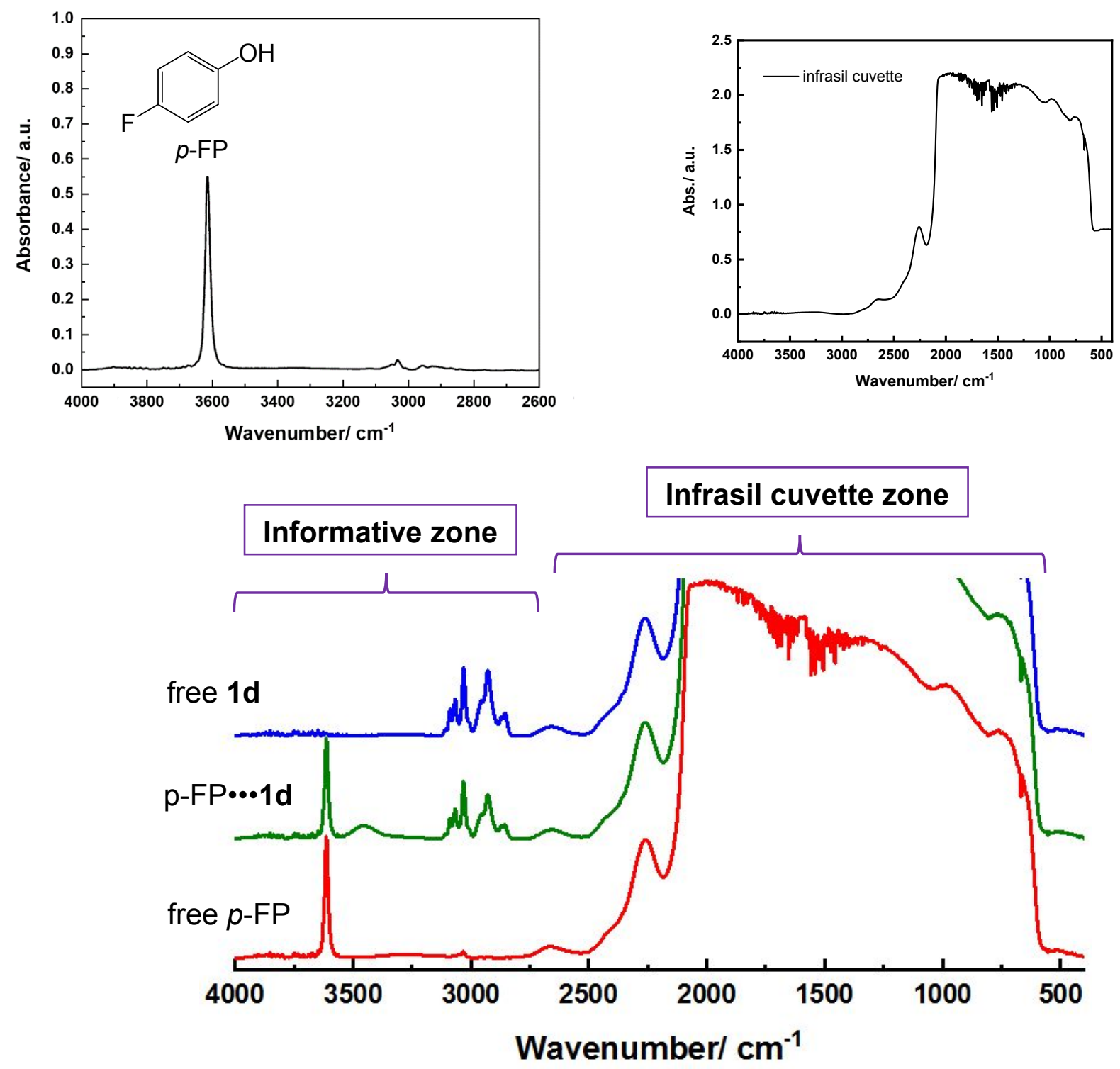

Figure S1. IR spectrum of 4-fluorophenol in dry $\mathrm{CCl}_{4}$ (top, left), IR spectrum of Infrasil cuvette (top, right) and an example of full IR range analysis of free 1d, complexed $p$-FP with $\mathbf{1 d}$ and free $p$-FP (bottom). 


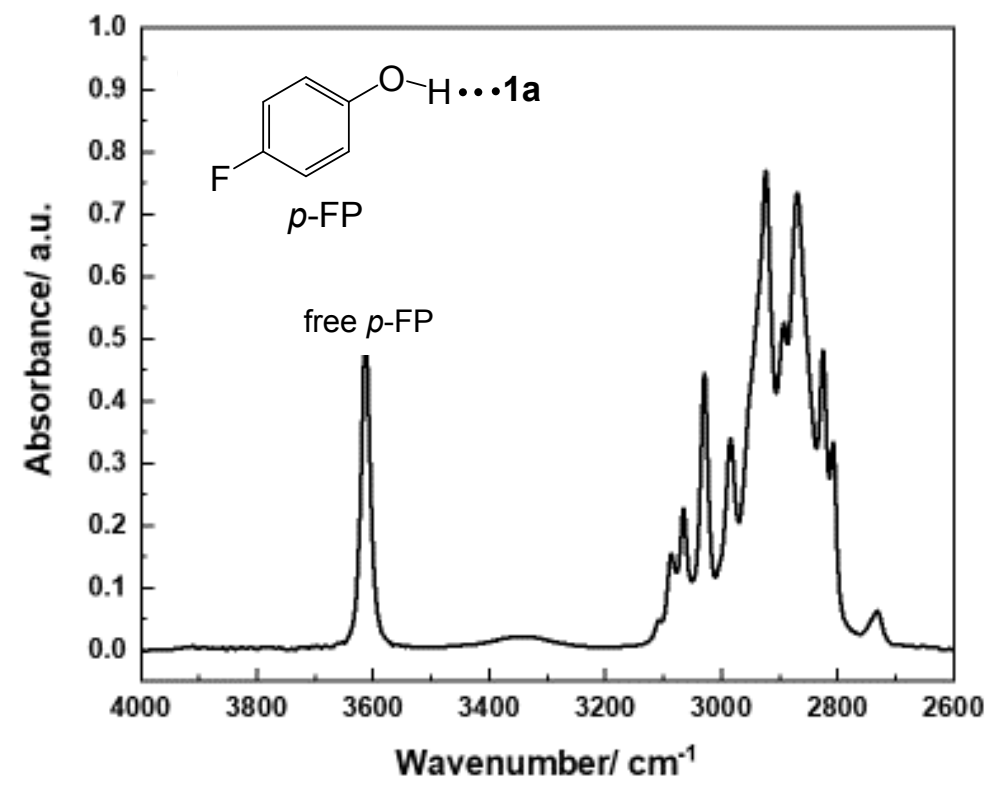

Figure S2. IR spectrum of the association of 4-fluorophenol with compound 1a.

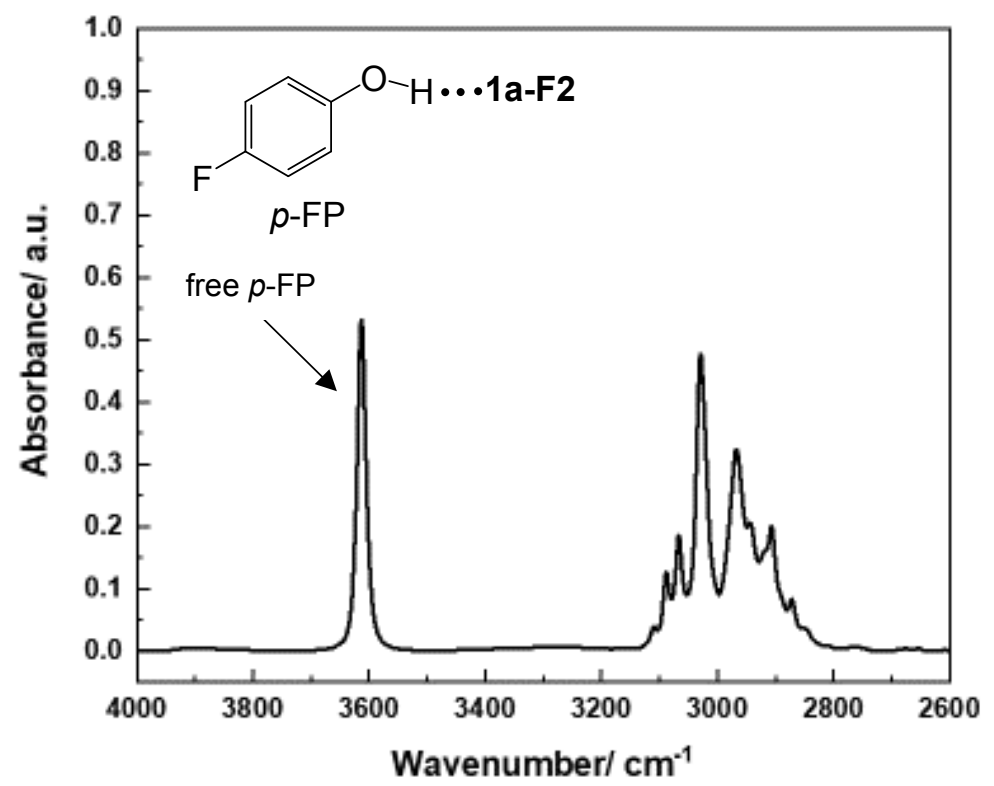

Figure S3. IR spectrum of the association of 4-fluorophenol with compound 1a-F2. 


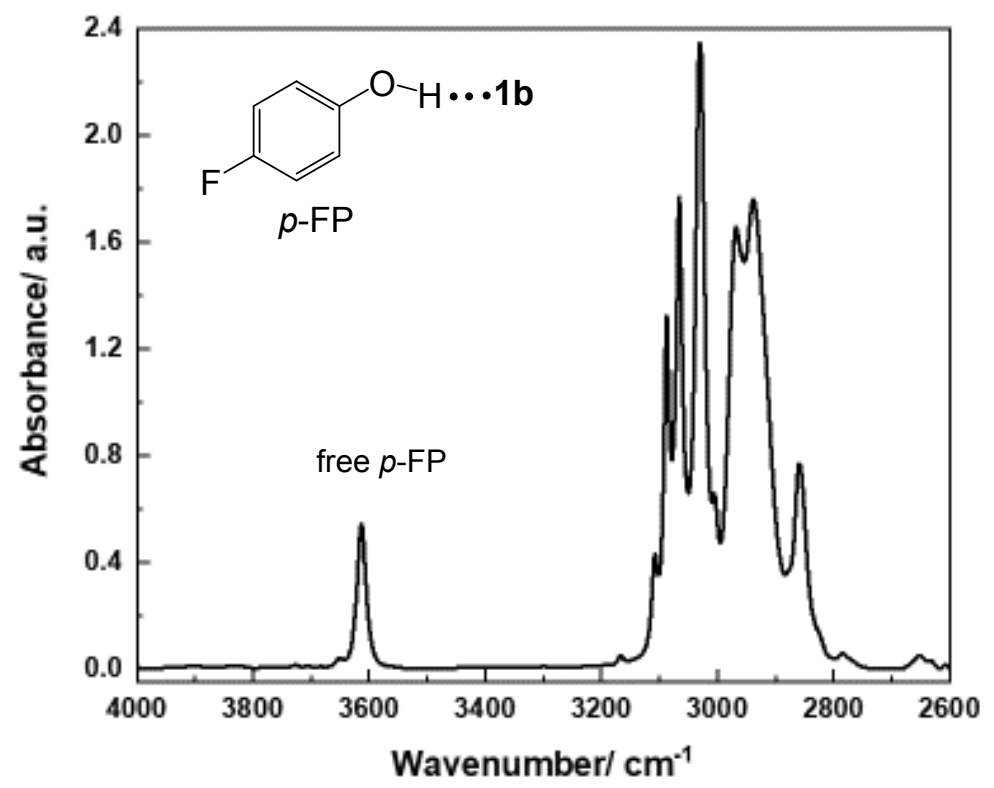

Figure S4. IR spectrum of the association of 4-fluorophenol with compound $\mathbf{1 b}$.

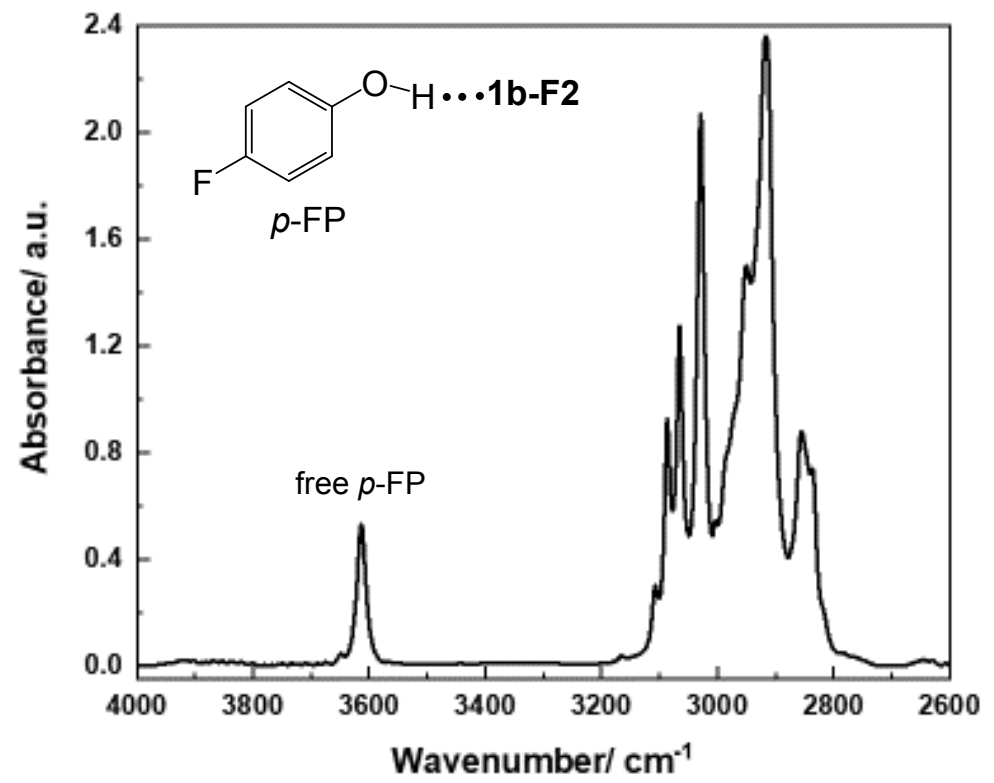

Figure S5. IR spectrum of the association of 4-fluorophenol with compound 1b-F2. 


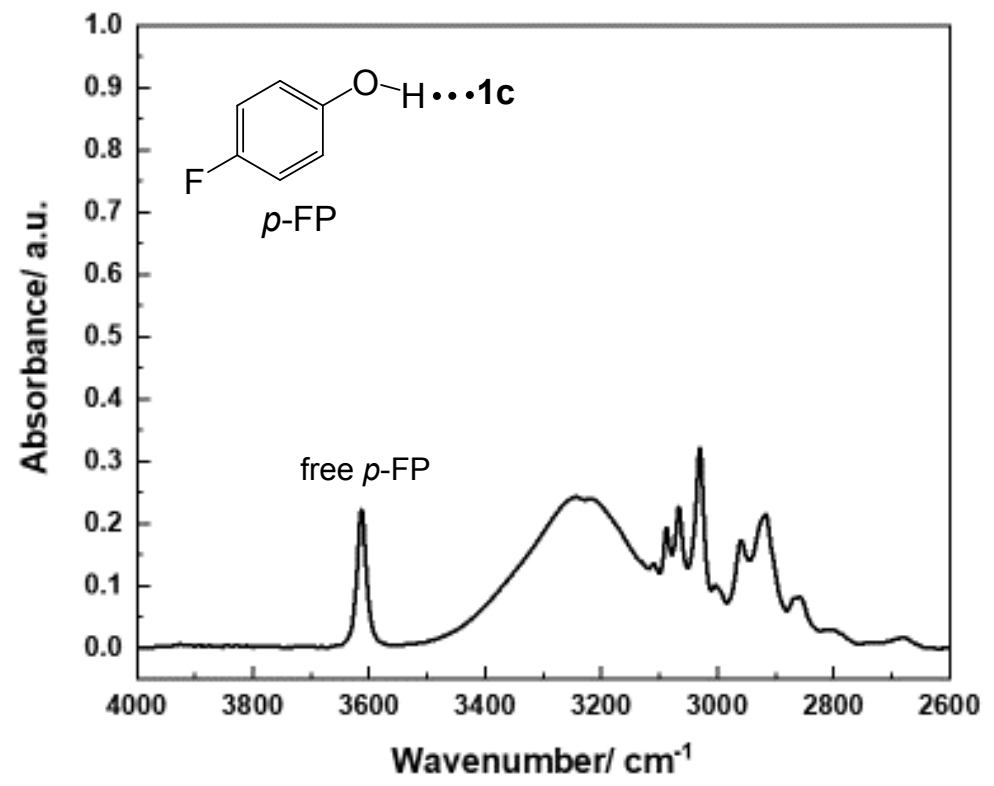

Figure S6. IR spectrum of the association of 4-fluorophenol with compound 1c.

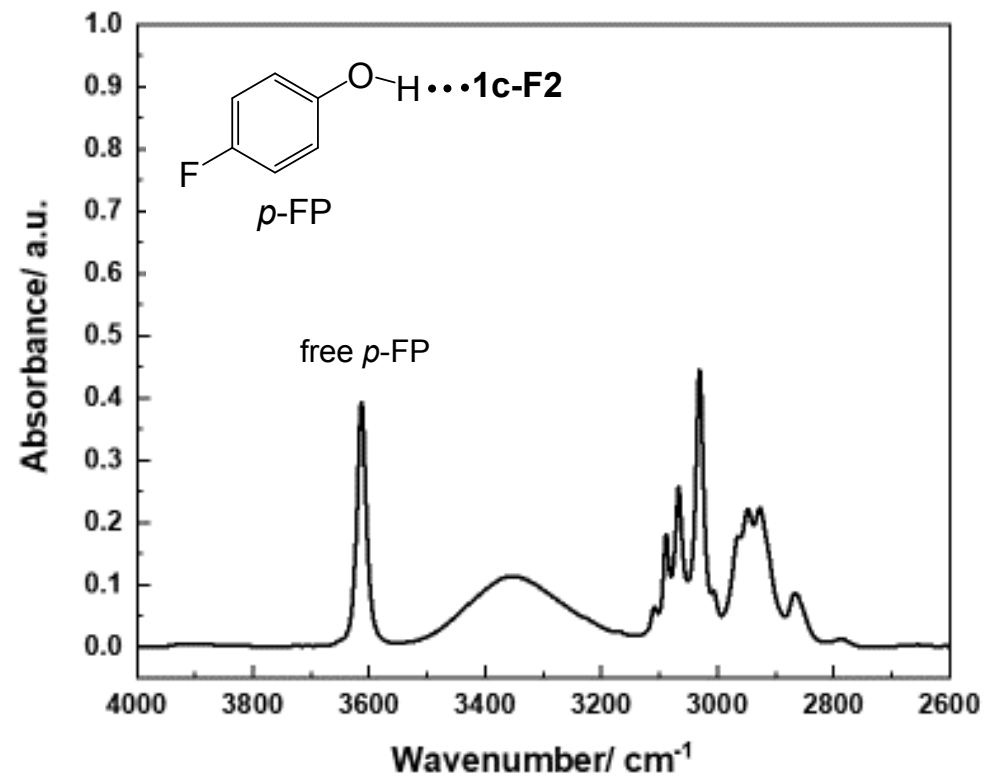

Figure S7. IR spectrum of the association of 4-fluorophenol with compound 1c-F2. 


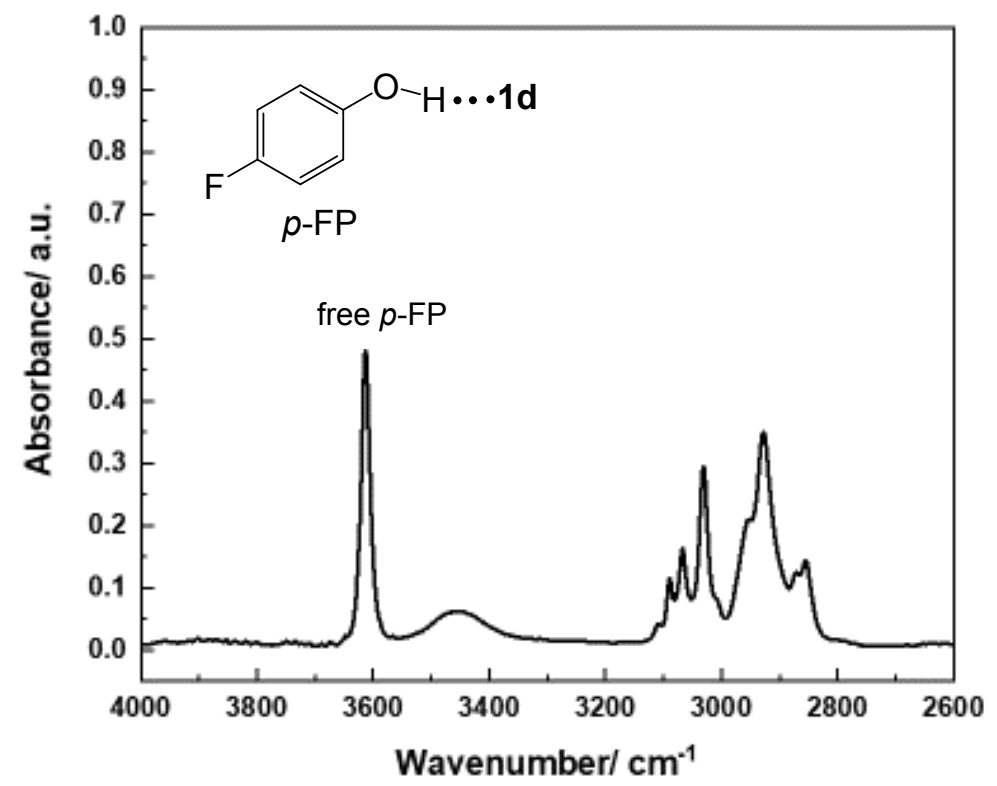

Figure S8. IR spectrum of the association of 4-fluorophenol with compound 1d.

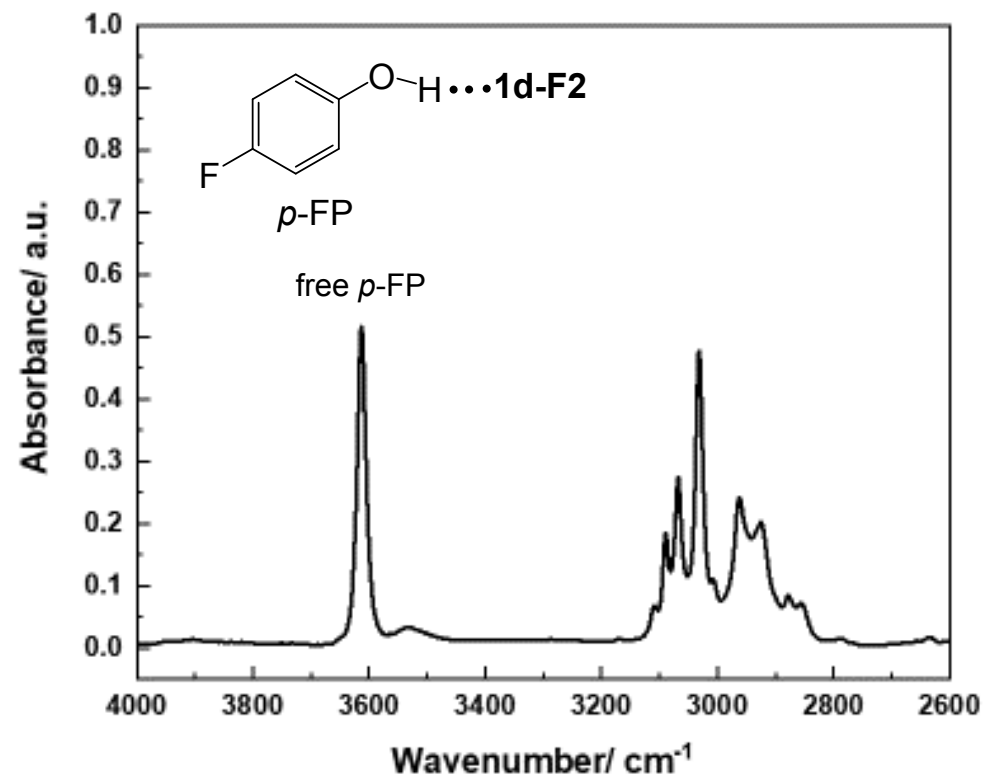

Figure S9. IR spectrum of the association of 4-fluorophenol with compound 1d-F2. 


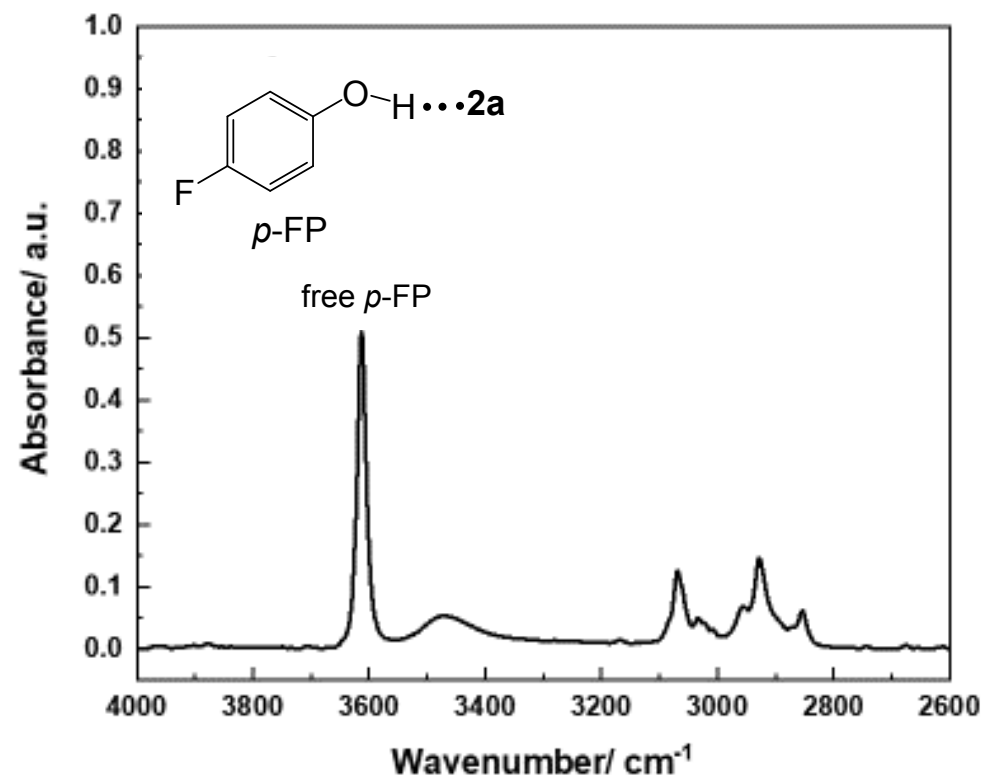

Figure S10. IR spectrum of the association of 4-fluorophenol with compound 2a.

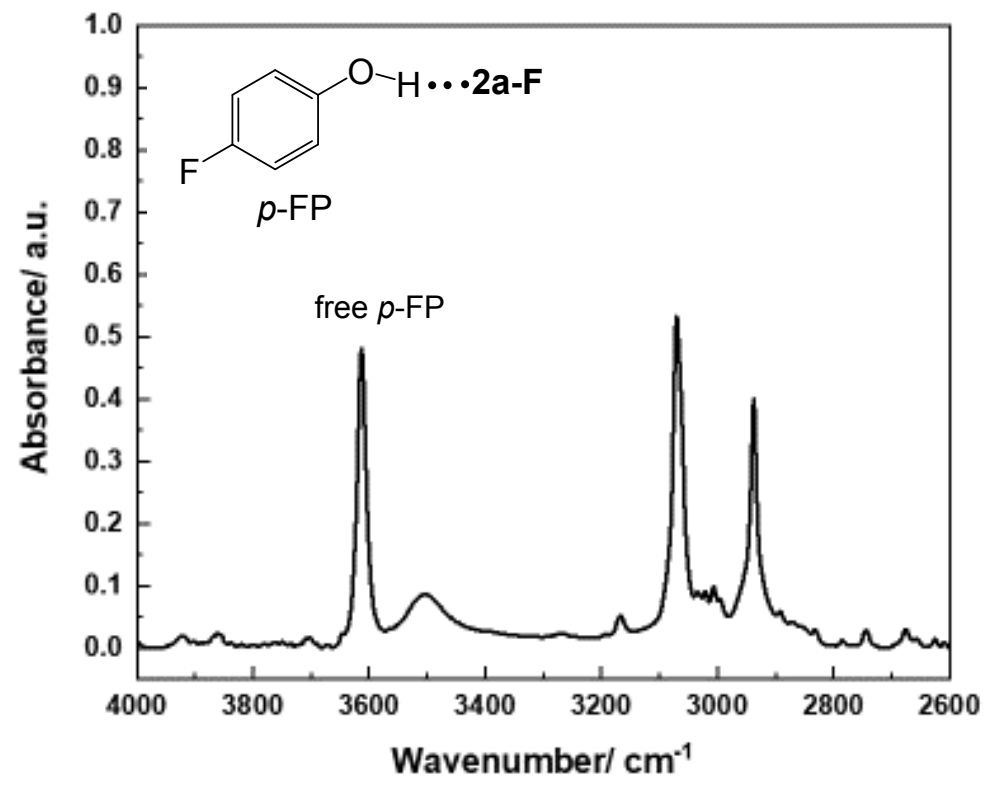

Figure S11. IR spectrum of the association of 4-fluorophenol with compound 2a-F. 


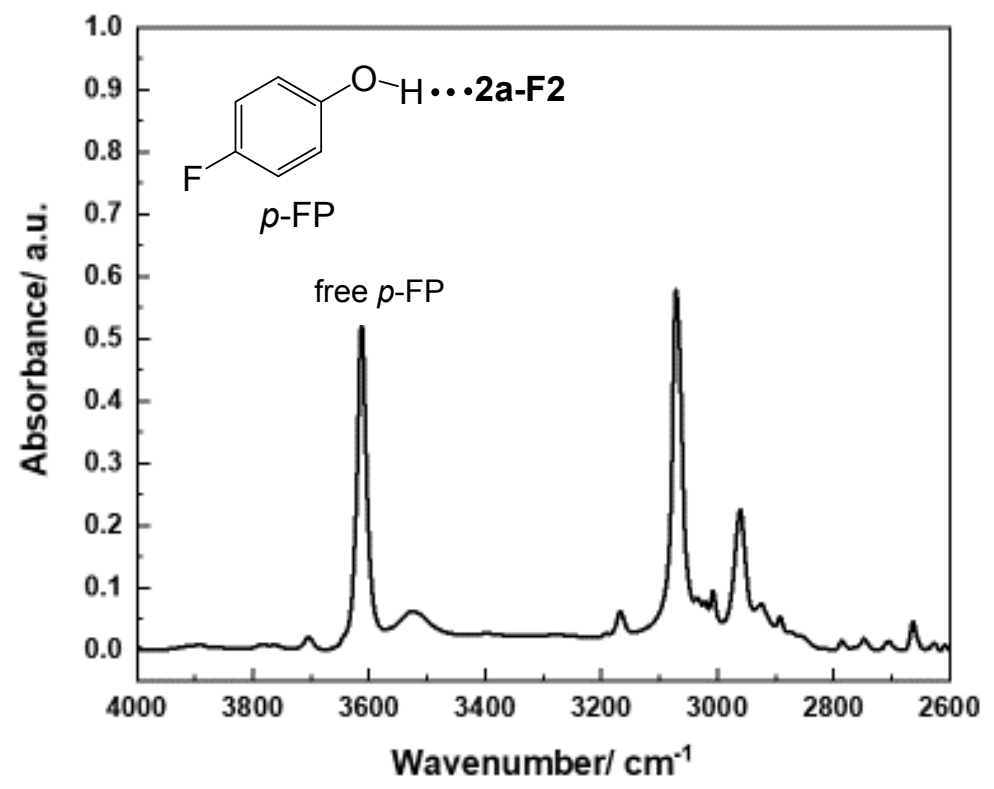

Figure S12. IR spectrum of the association of 4-fluorophenol with compound 2a-F2.

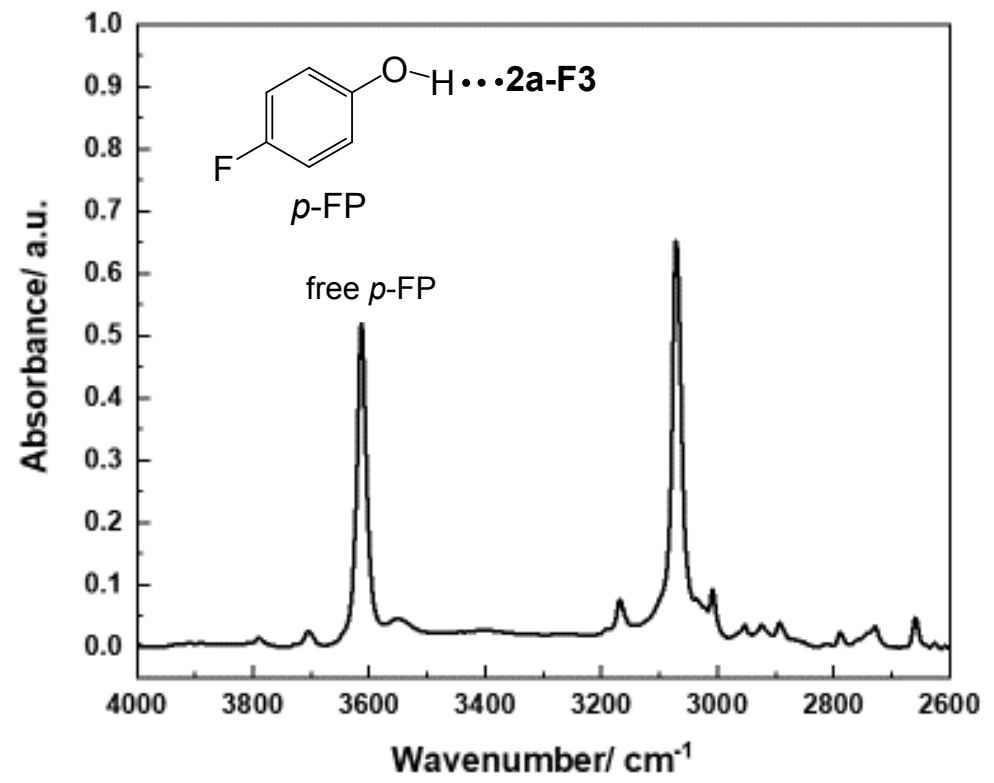

Figure S13. IR spectrum of the association of 4-fluorophenol with compound 2a-F3. 


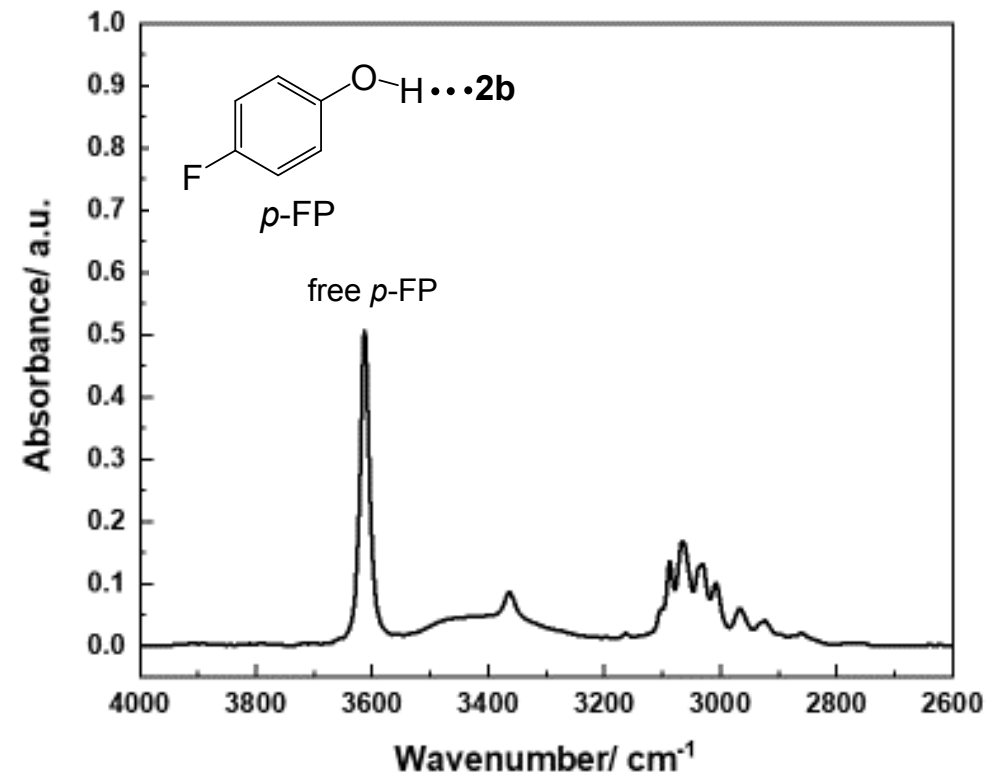

Figure S14. IR spectrum of the association of 4-fluorophenol with compound $\mathbf{2 b}$.

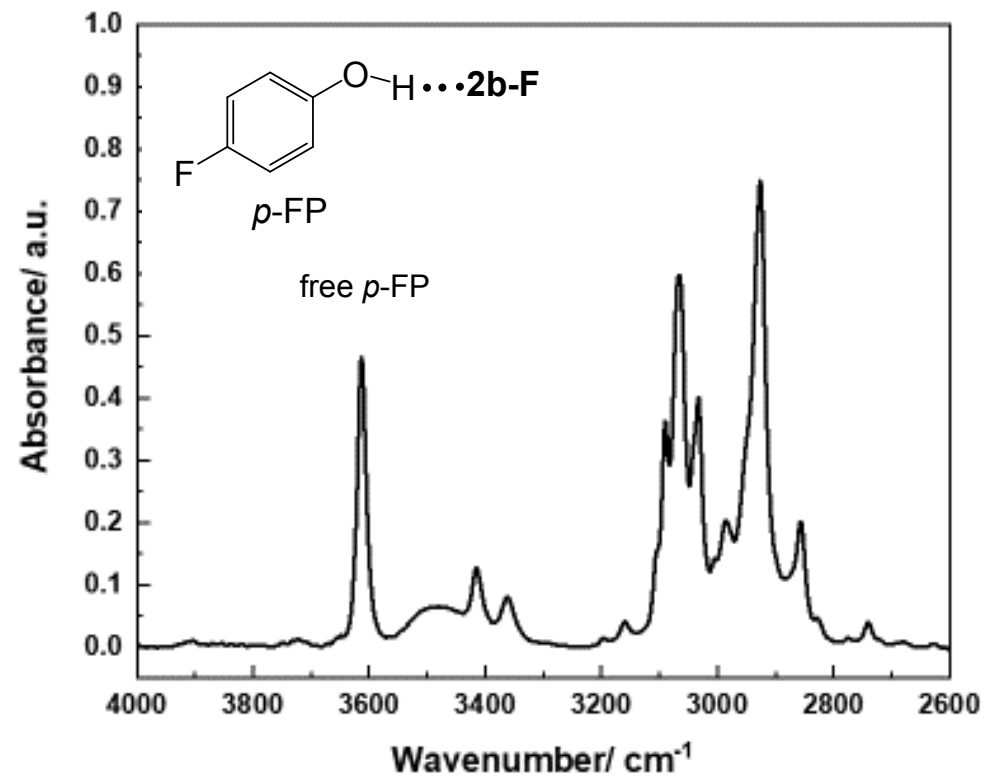

Figure S15. IR spectrum of the association of 4-fluorophenol with compound $\mathbf{2 b - F}$. 


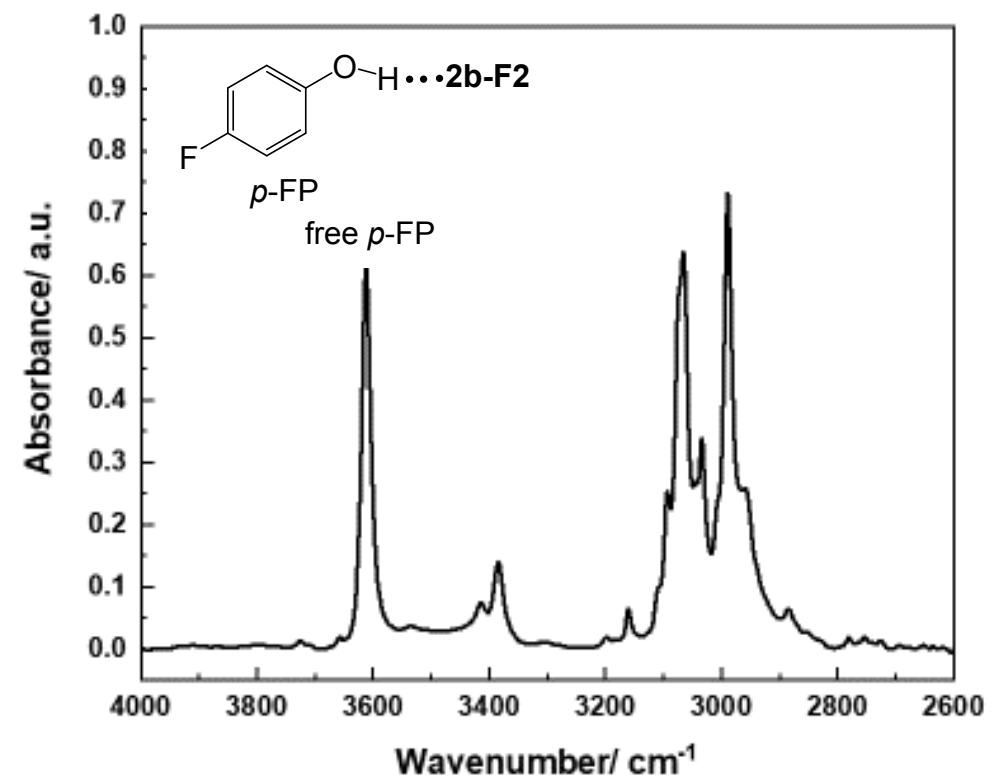

Figure S16. IR spectrum of the association of 4-fluorophenol with compound $2 \mathbf{b}-\mathbf{F} 2$.

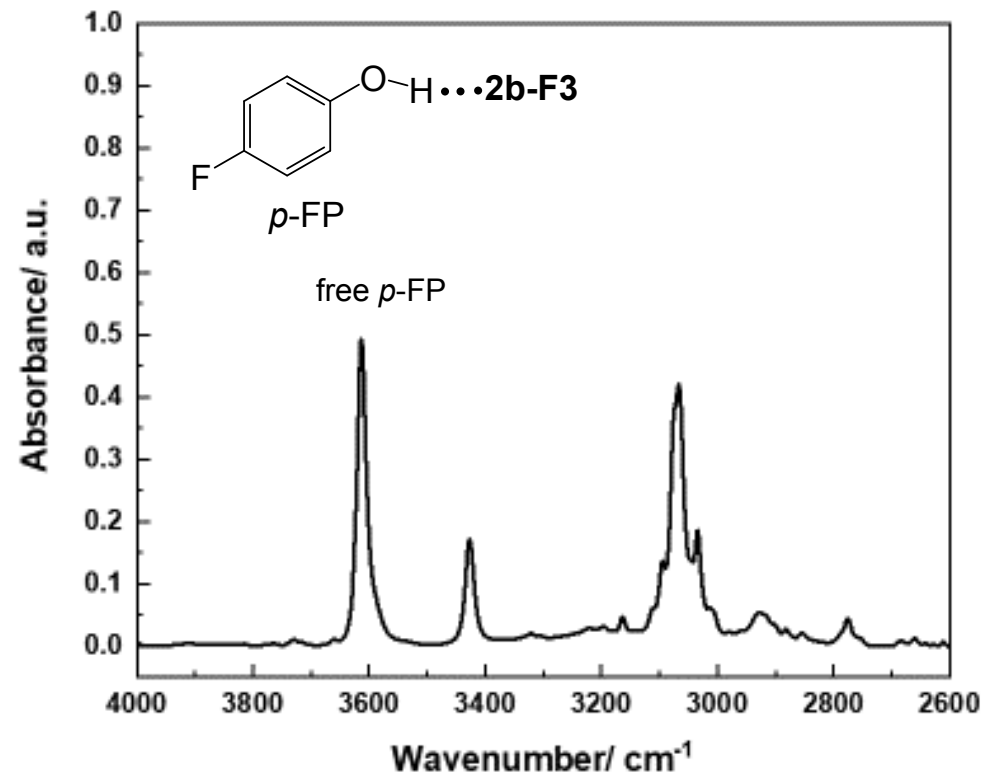

Figure S17. IR spectrum of the association of 4-fluorophenol with compound 2b-F3. 


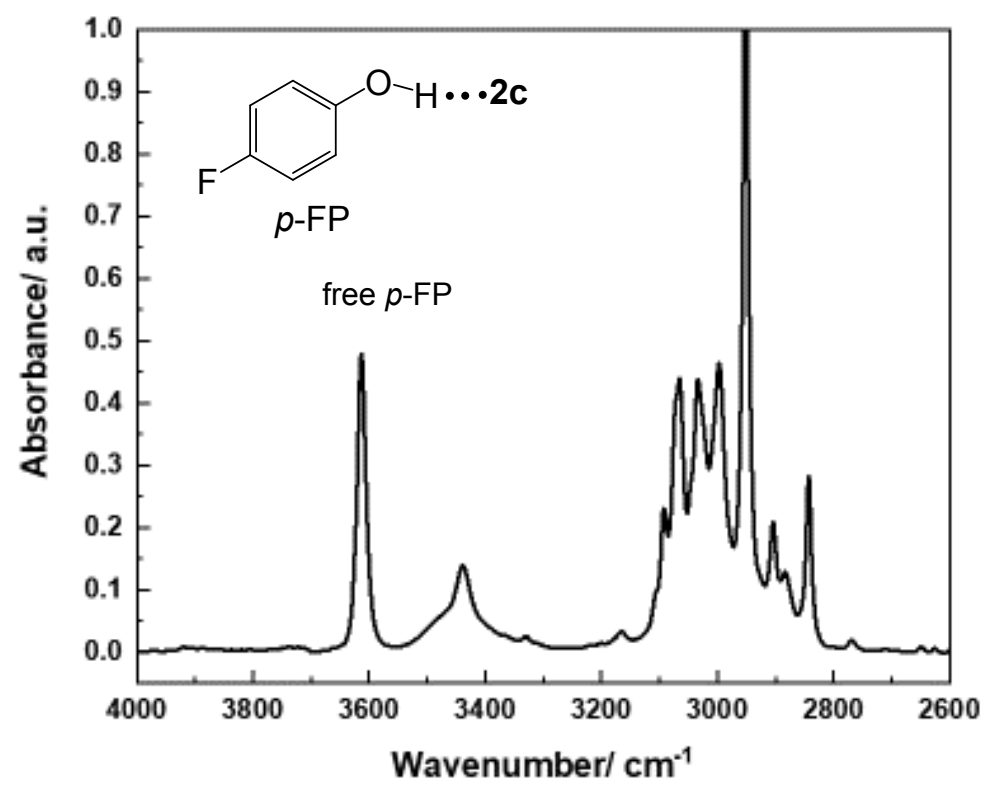

Figure S18. IR spectrum of the association of 4-fluorophenol with compound 2c.

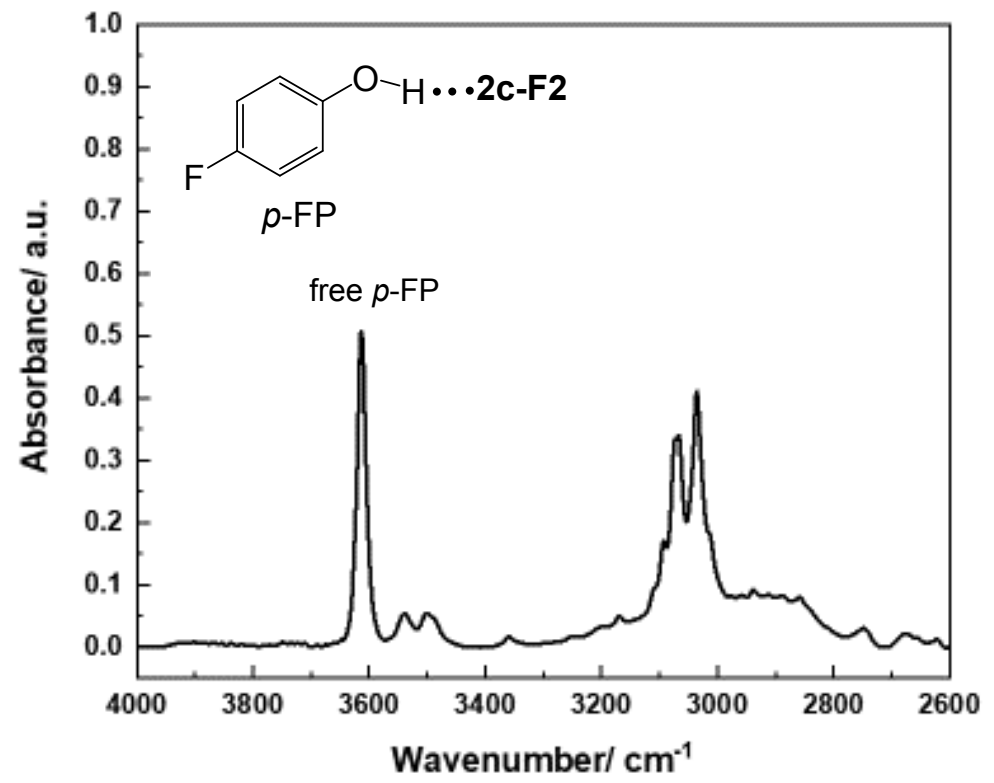

Figure S19. IR spectrum of the association of 4-fluorophenol with compound 2c-F2. 


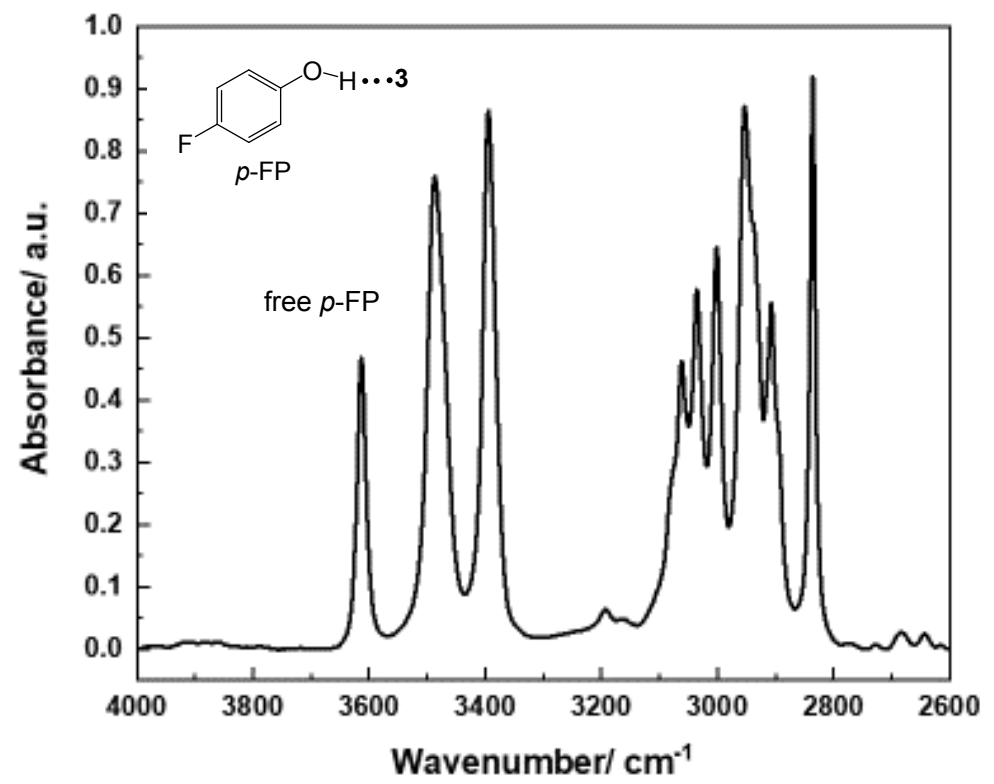

Figure S20. IR spectrum of the association of 4-fluorophenol with compound 3.

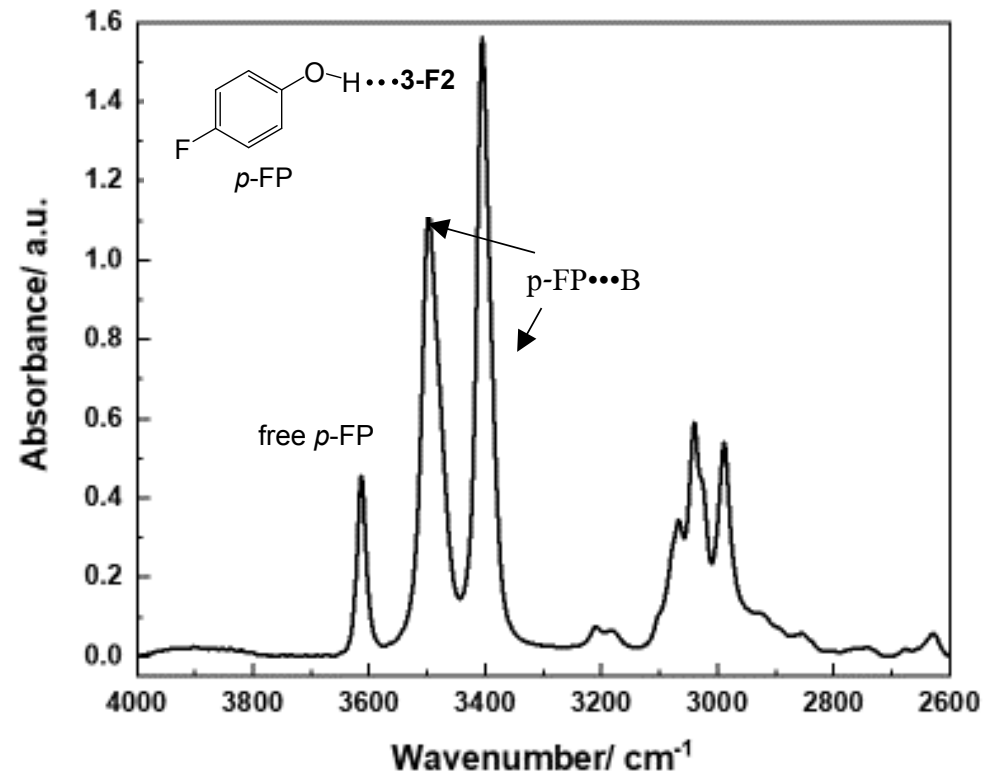

Figure S21. IR spectrum of the association of 4-fluorophenol with compound 3-F2. 


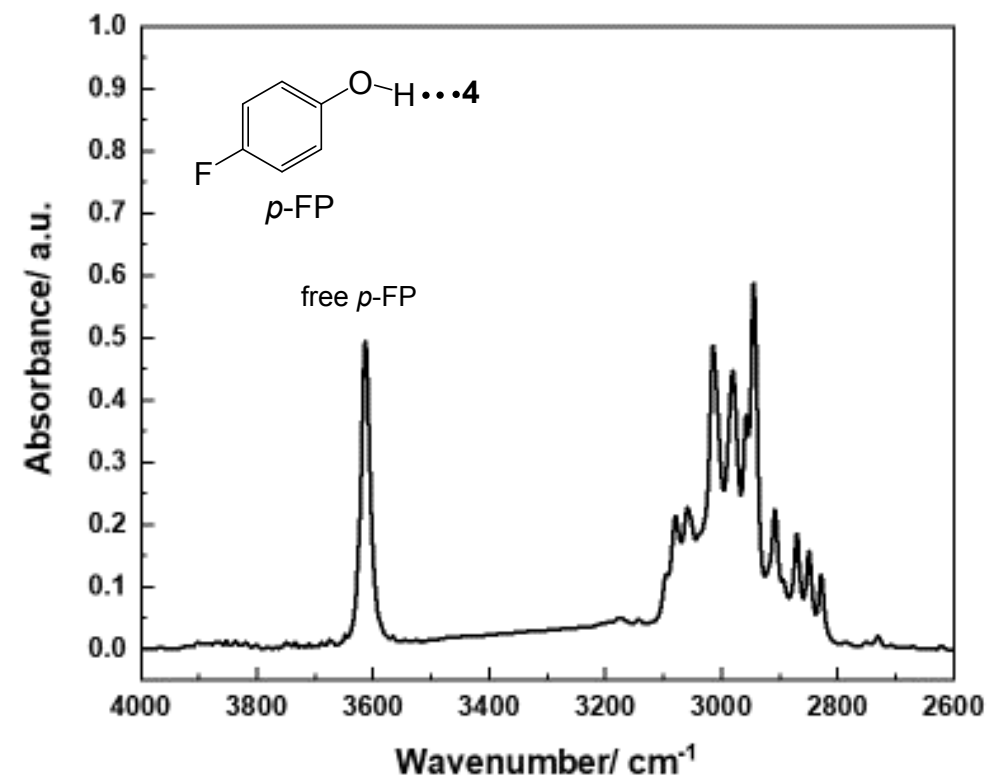

Figure S22. IR spectrum of the association of 4-fluorophenol with compound 4.

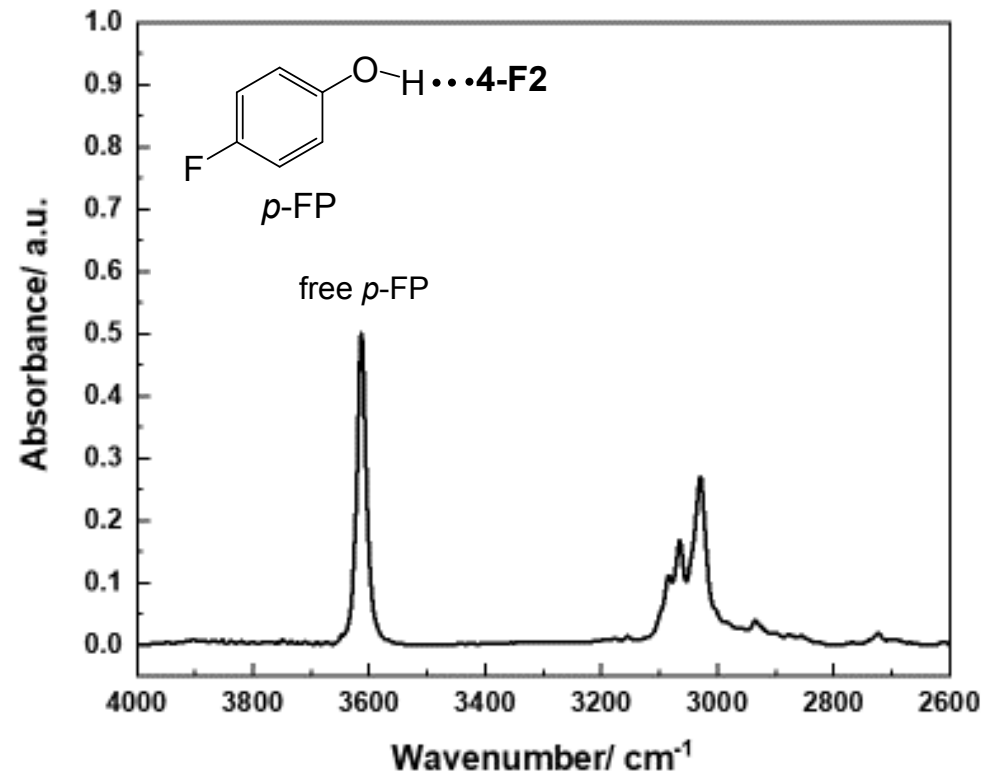

Figure S23. IR spectrum of the association of 4-fluorophenol with compound 4-F2. 


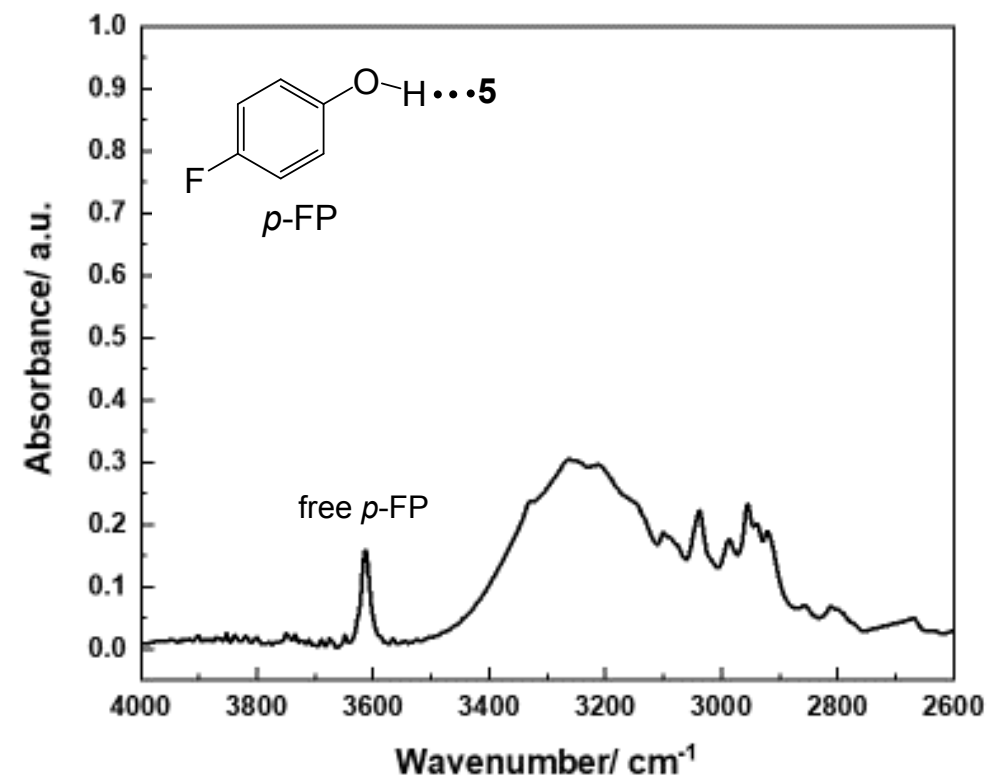

Figure S24. IR spectrum of the association of 4-fluorophenol with compound 5.

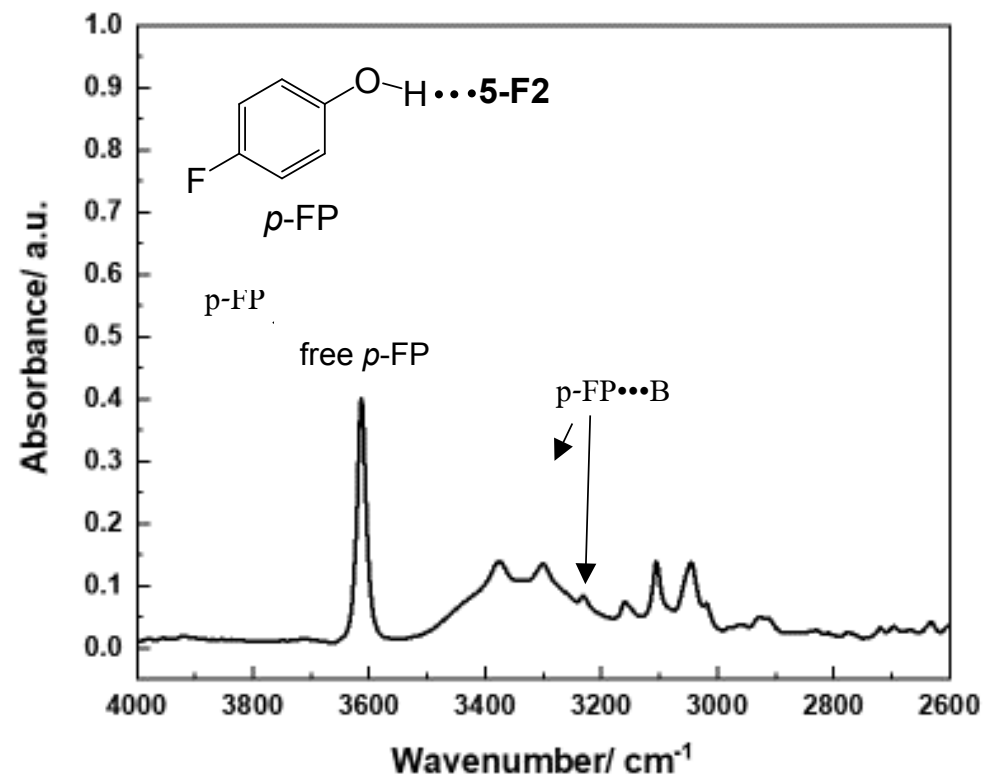

Figure S25. IR spectrum of the association of 4-fluorophenol with compound 5-F2. 


\section{$\underline{1.1 .2 \mathrm{pK}} \underline{\mathrm{HB}} \underline{\text { measurements by }{ }^{19} \mathrm{~F}-\mathrm{NMR}}$}

$\mathrm{p} K_{\mathrm{HB}}$ was calculated according to Eqn 1:

$K_{f=}\left(\frac{\delta}{\Delta \max }\right) A o /\left\{A o\left[1-\left(\frac{\delta}{\Delta \max }\right)\right]\right\}\left[B o-\left(\frac{\delta}{\Delta \max }\right) A o\right]$

Table 2 presents for example the calculation of acetophenone which gave $\mathrm{p} K_{\mathrm{HB}} 1.22 \pm 0.02$.

Table S2. Formation constant of the p-FP-acetophenone complex in $\mathrm{CCl}_{4}$ at $25{ }^{\circ} \mathrm{C}$.

\begin{tabular}{lll}
\hline$A_{0}$ & $B_{0}$ & $\delta$ observed $^{b}$ \\
\hline 10.85 & 13.70 & 0.283 \\
11.04 & 7.43 & 0.165 \\
11.16 & 3.48 & 0.075 \\
10.69 & 19.36 & 0.376 \\
10.92 & 11.64 & 0.254 \\
9.78 & 86.70 & 0.977 \\
10.45 & 28.18 & 0.557 \\
10.80 & 242.66 & 1.568 \\
\hline
\end{tabular}

${ }^{a}$ All concentrations are in $\mathrm{mmol} \mathrm{L}^{-1} .{ }^{b} \delta$ in ppm. $\Delta_{\max }=1.71 \mathrm{ppm}$. 


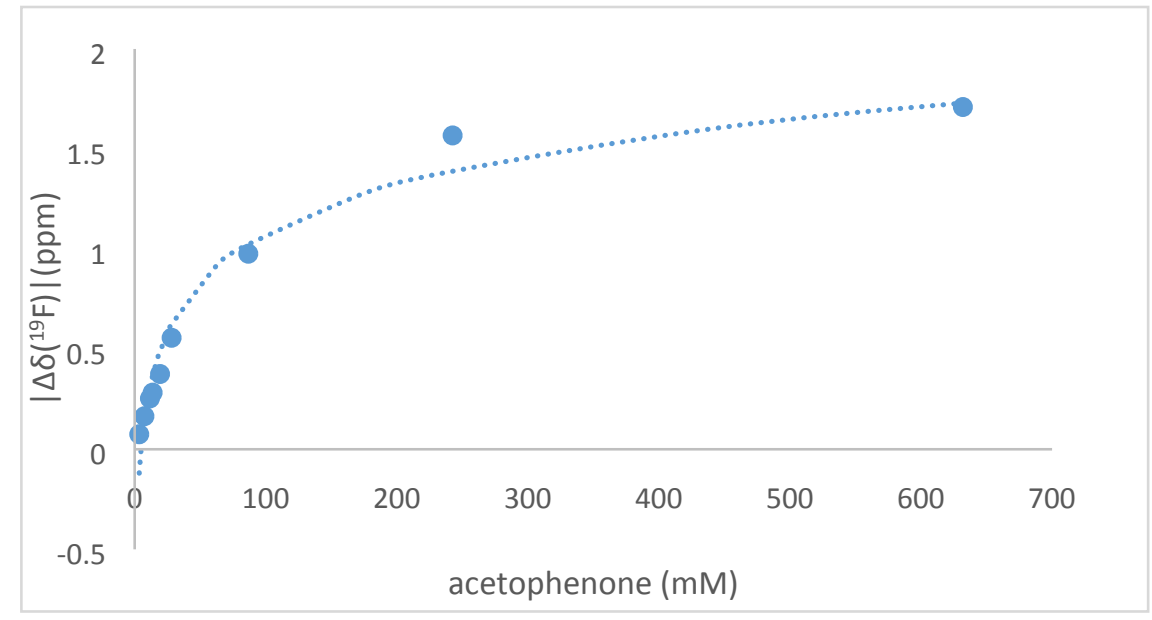

Figure S26. Plot of $\left|\Delta \delta\left({ }^{19} \mathrm{~F}\right)\right|$ as a function of acetophenone concentration.

$\left|\Delta \delta\left({ }^{19} \mathrm{~F}\right)\right|=\Delta \delta_{\mathrm{A}}\left({ }^{19} \mathrm{~F}\right)-\Delta \delta_{0}\left({ }^{19} \mathrm{~F}\right)$ with $\Delta \delta_{\mathrm{A}}\left({ }^{19} \mathrm{~F}\right)$ and $\Delta \delta_{0}\left({ }^{19} \mathrm{~F}\right)$ being the chemical shift difference between the ${ }^{19} \mathrm{~F}$ signals of $\mathrm{p}$-FP and of $\mathrm{p}$-fluoroanisole, in the presence and absence of acetophenone, respectively. 


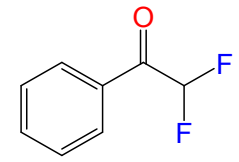

$\mathbf{2 b - F 2}$ in octanol saturated with water
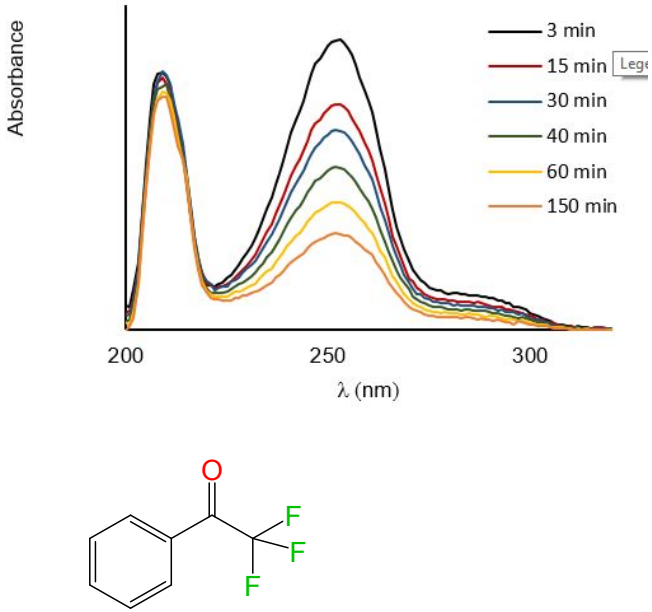

2b-F3 in octanol saturated with water

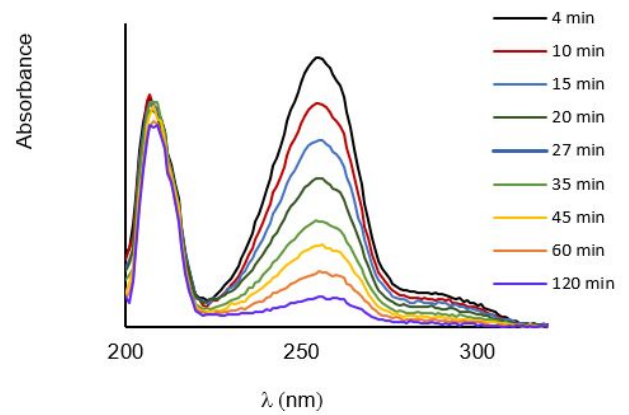

Figure S27. The decay of the UV-absorption of fluorinated ketones $\mathbf{2 b}-\mathbf{F} 2$ (up) and $\mathbf{2 b - F 3}$ (down) in octanol saturated with water. 


\section{$\underline{\text { 2. }{ }^{19} \text { F NMR spectra }}$}

$\underline{2.1{ }^{19} \mathrm{~F} \text { NMR spectra of } 2 \mathrm{~b}-\mathrm{F} 2 \text { in octanol (sat. with water) and in } \mathrm{H}_{2}} \underline{\mathrm{O} \text { (sat. with oct.) }}$

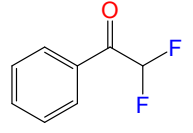

2b-F2

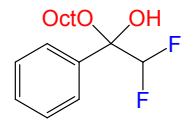

6

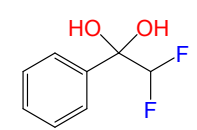

7
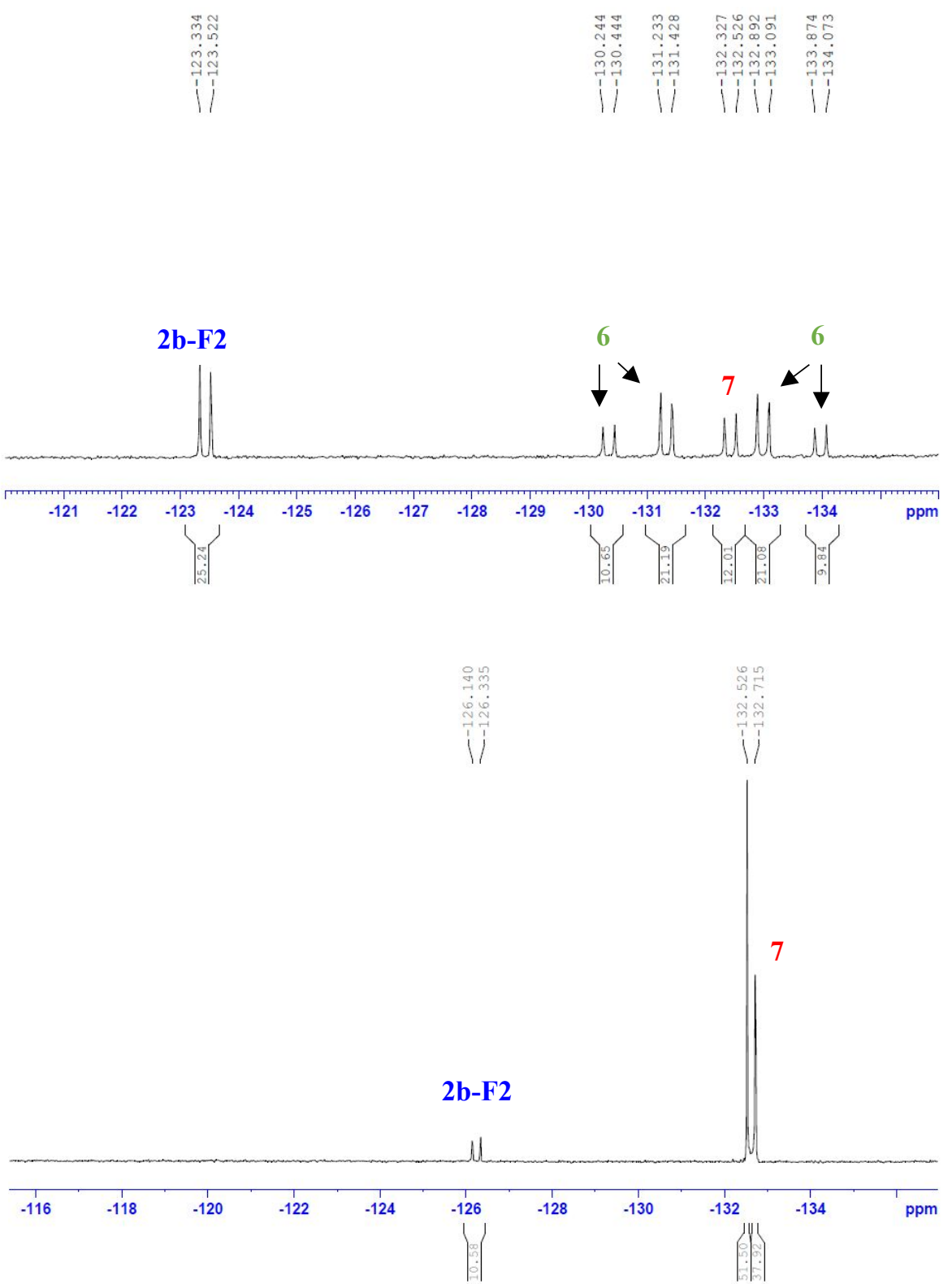

Figure S28. ${ }^{19}$ F NMR spectra of 2b-F2 in octanol saturated with water (top) and in $\mathrm{H}_{2} \mathrm{O}$ (down). 
$\underline{2.2}{ }^{19} \mathrm{~F}$ NMR spectra of $2 \mathrm{~b}-\mathrm{F} 3$ in octanol (sat. with water) and in $\mathrm{H}_{2} \underline{\mathrm{O} \text { (sat. with oct.) }}$<smiles>CCPC(F)(P)C(=O)c1ccccc1</smiles>

2b-F3

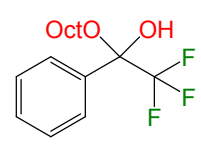

8

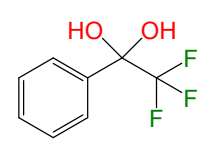

9

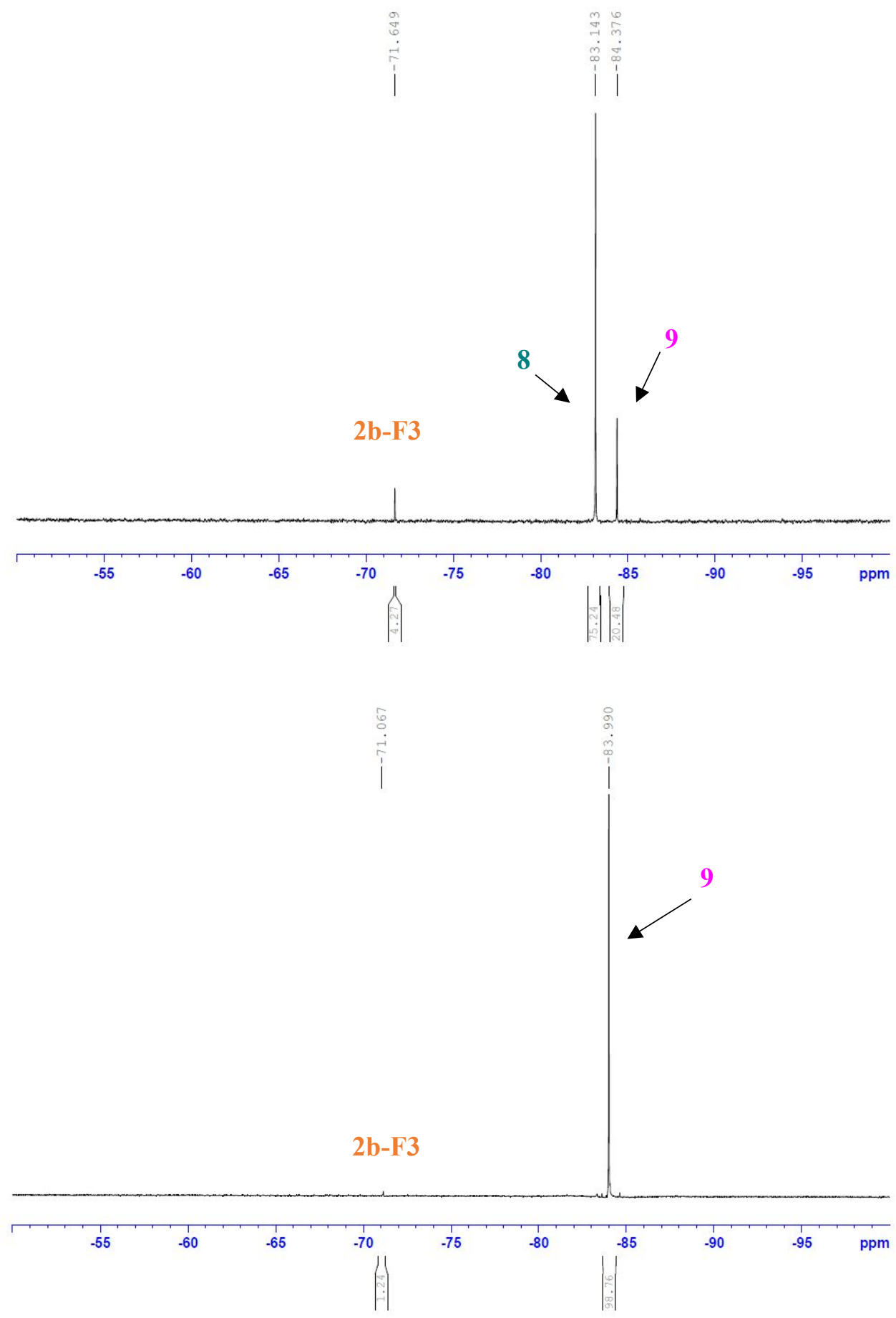

Figure S29. ${ }^{19} \mathrm{~F}$ NMR spectra of $\mathbf{2 b - F 3}$ in octanol saturated with water (top) and in $\mathrm{H}_{2} \mathrm{O}$ (down). 


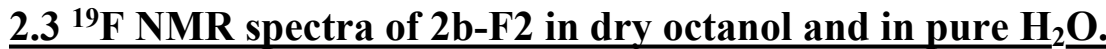

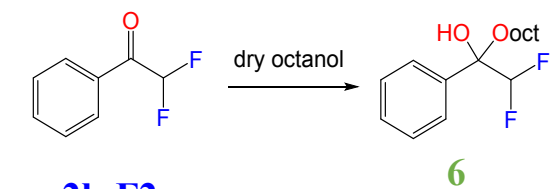

2b-F2
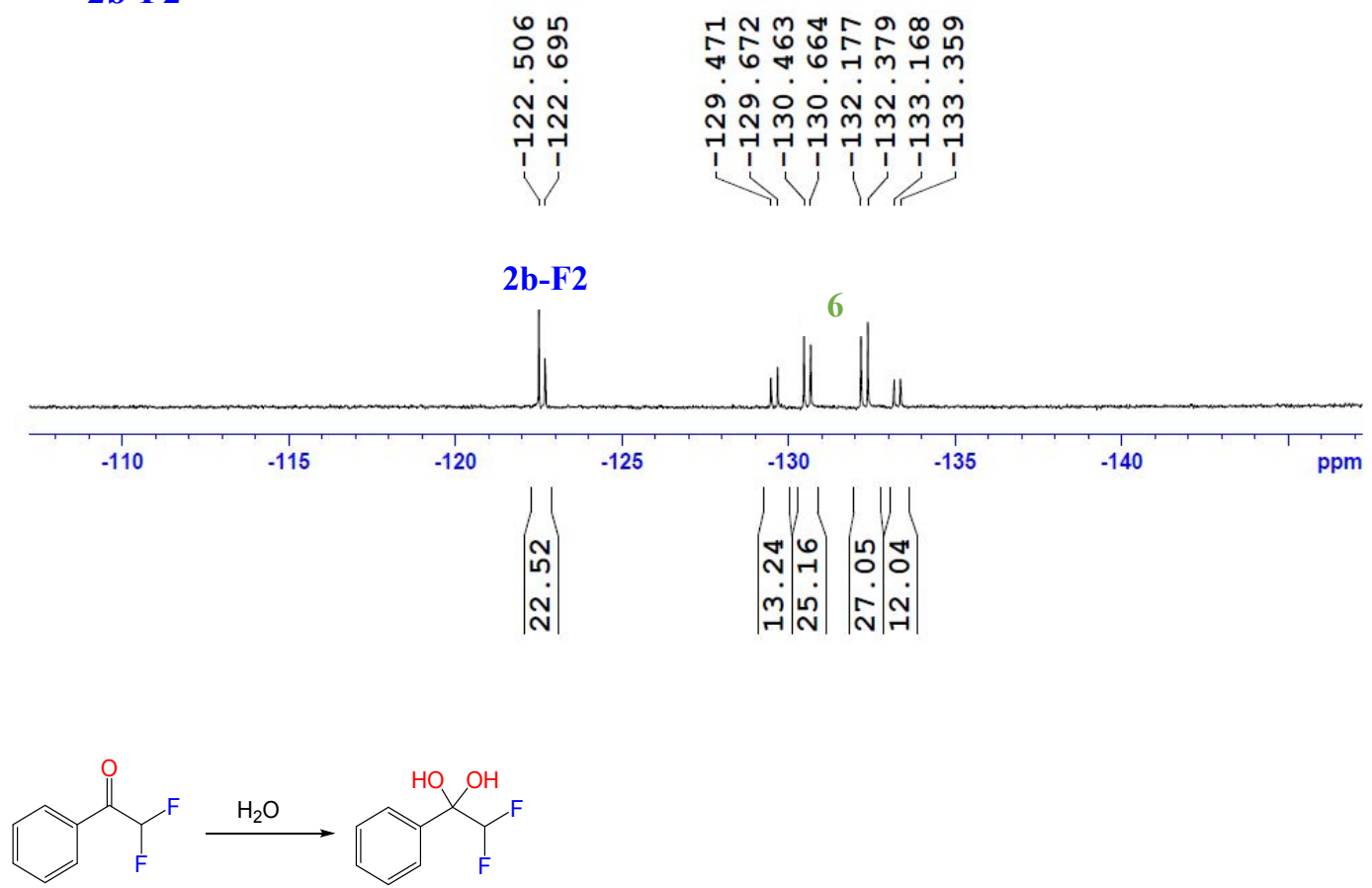

2b-F2

7

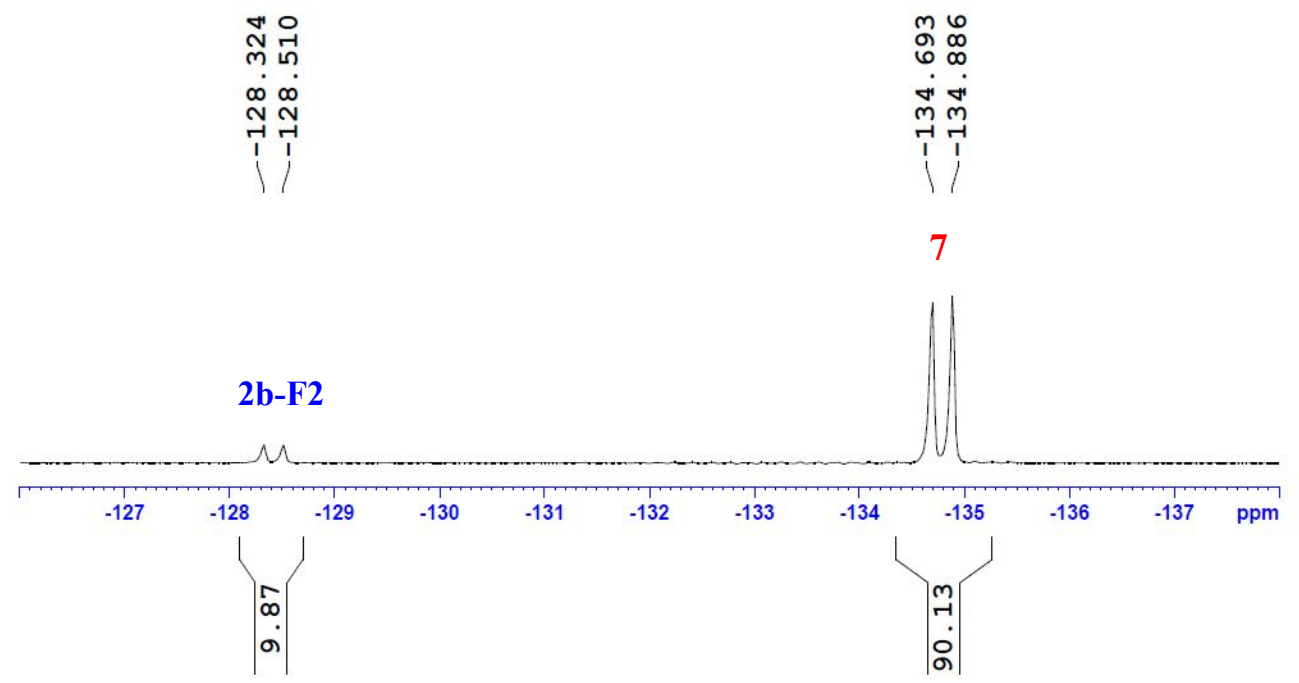

Figure S30. ${ }^{19} \mathrm{~F}$ NMR spectra of $\mathbf{2 b}-\mathbf{F} 2$ in dry octanol (top) and in pure $\mathrm{H}_{2} \mathrm{O}$ (down). 
$\underline{2.4{ }^{19} \mathrm{~F} \text { NMR spectra of } 2 \mathrm{~b}-\mathrm{F} 3 \text { in dry octanol and in pure } \mathrm{H}_{2}} \underline{\mathrm{O}}$.
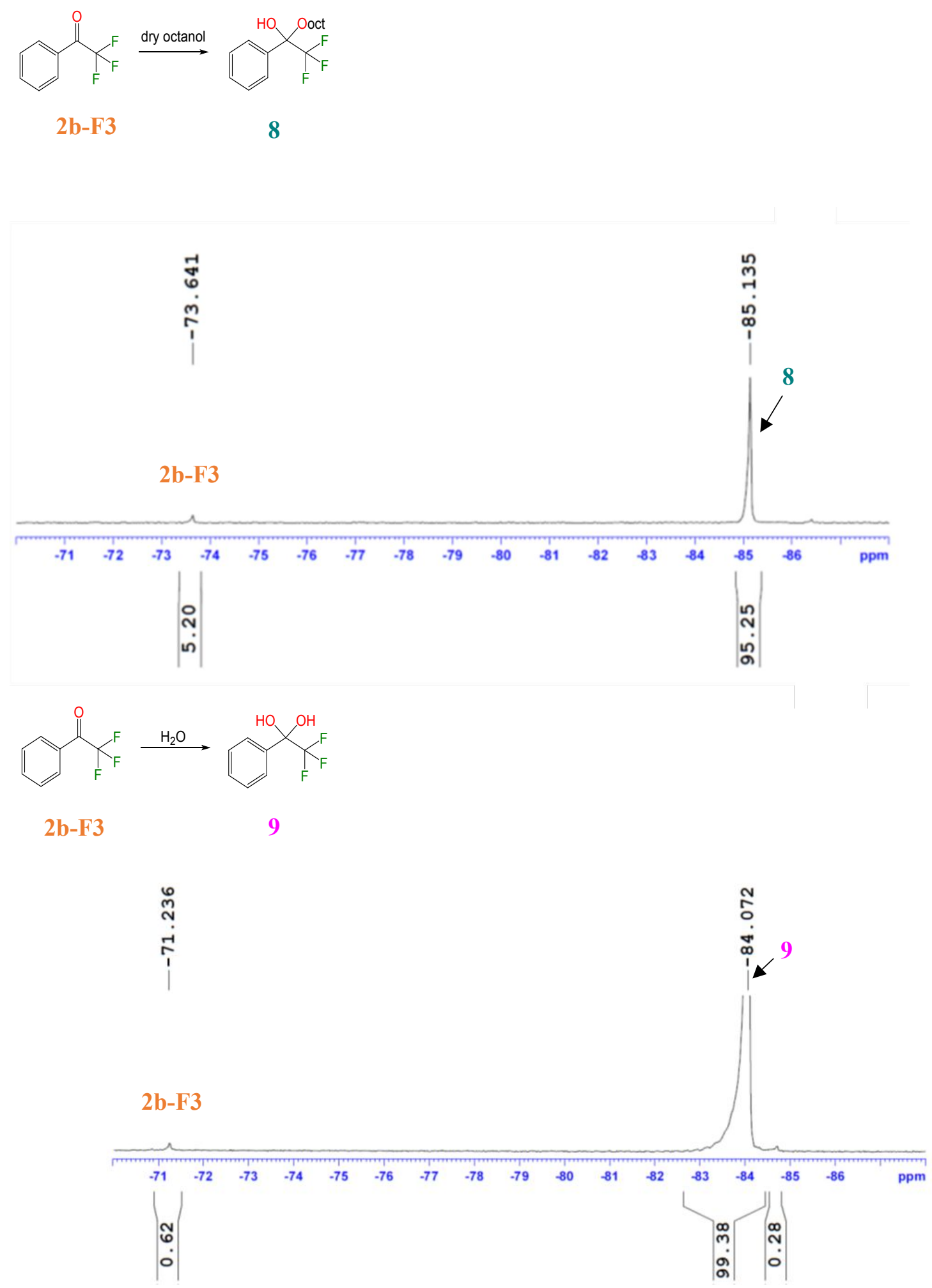

Figure S31. ${ }^{19} \mathrm{~F}$ NMR spectra of 2b-F3 in dry octanol (top) and in pure $\mathrm{H}_{2} \mathrm{O}$ (down). 


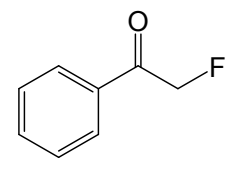

2b-F

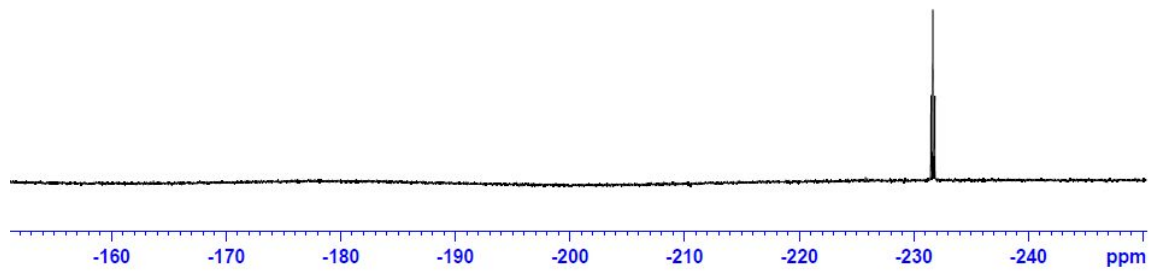

Figure S32. ${ }^{19}$ F NMR spectra of $\mathbf{2 b}-\mathbf{F}$ in octanol saturated with $\mathrm{H}_{2} \mathrm{O}$. 
<smiles>O=C(CF)c1ccccc1</smiles>

2b-F
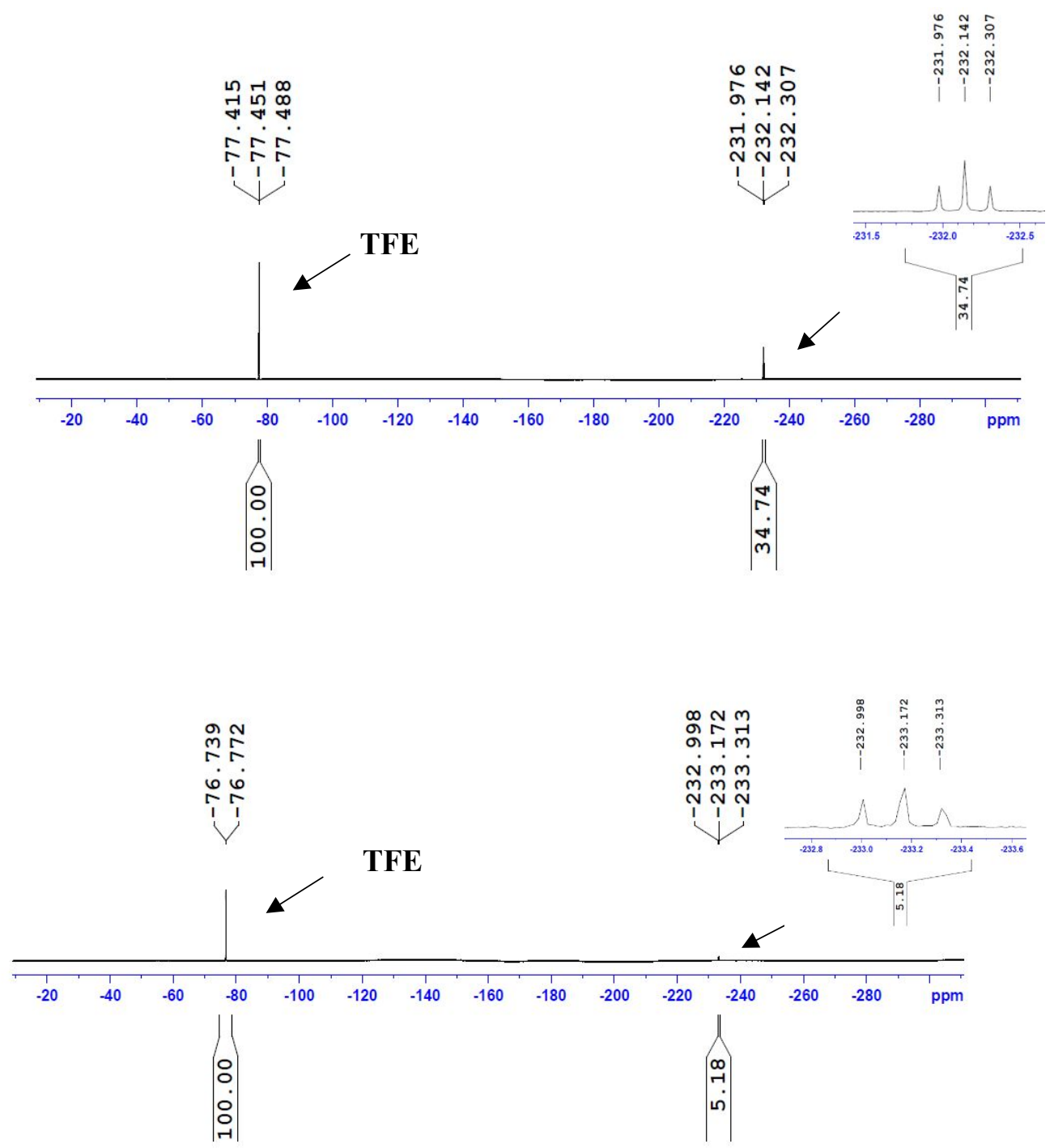

Figure S33. ${ }^{19} \mathrm{~F}$ NMR spectra of $\mathbf{2 b - F}$ in octanol (top) $\mathrm{H}_{2} \mathrm{O}$ (down) after the separation of the phases for $\log P_{\mathrm{o} / \mathrm{w}}$ determination. 


\section{$\underline{2.7 \log P \text { determination of } 2 \mathrm{~b}-\mathrm{F} 2 \text { by }{ }^{19} \mathrm{~F} \text { NMR }}$}<smiles>O=C(c1ccccc1)C(F)F</smiles>

2b-F2<smiles>O=C(O)C(O)(c1ccccc1)C(F)F</smiles>

6<smiles>OC(O)(c1ccccc1)C(F)F</smiles>

7

(\# sample 1 out of three)
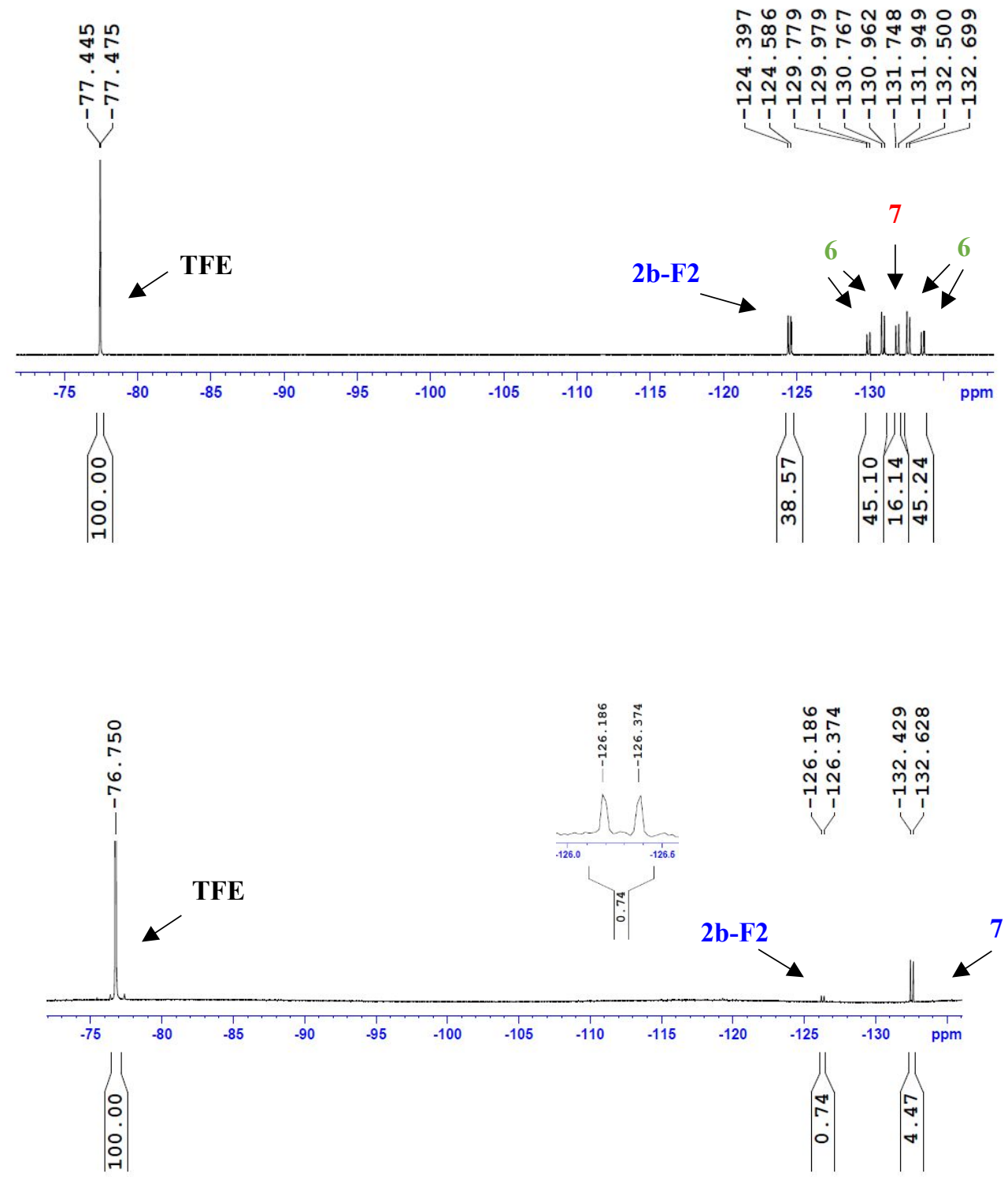

Figure S34. ${ }^{19} \mathrm{~F}$ NMR spectra of $\mathbf{2 b - F 2}$ in octanol (top) and $\mathrm{H}_{2} \mathrm{O}$ (down) after the separation of the phases for $\log P_{\mathrm{o} / \mathrm{w}}$ determination. 


\section{$\underline{2.8 \log P \text { determination of } 2 \mathrm{~b}-\mathrm{F} 3 \mathrm{by}{ }^{19} \mathrm{~F} \text { NMR }}$}<smiles>O=C(c1ccccc1)C(F)(F)F</smiles>

$2 \mathrm{~b}-\mathrm{F} 3$<smiles></smiles>

8<smiles>OC(O)(c1ccccc1)C(F)(F)F</smiles>

9

(\# sample 1 out of three)
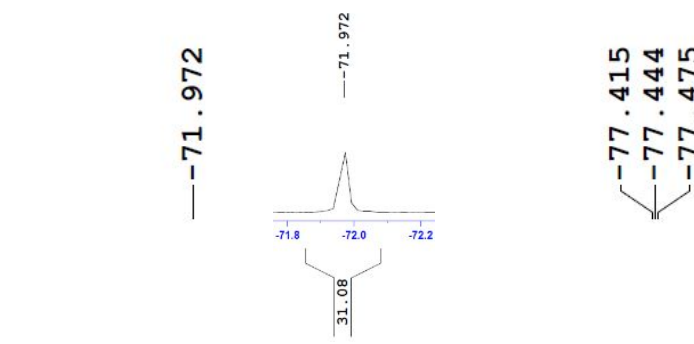

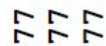

1
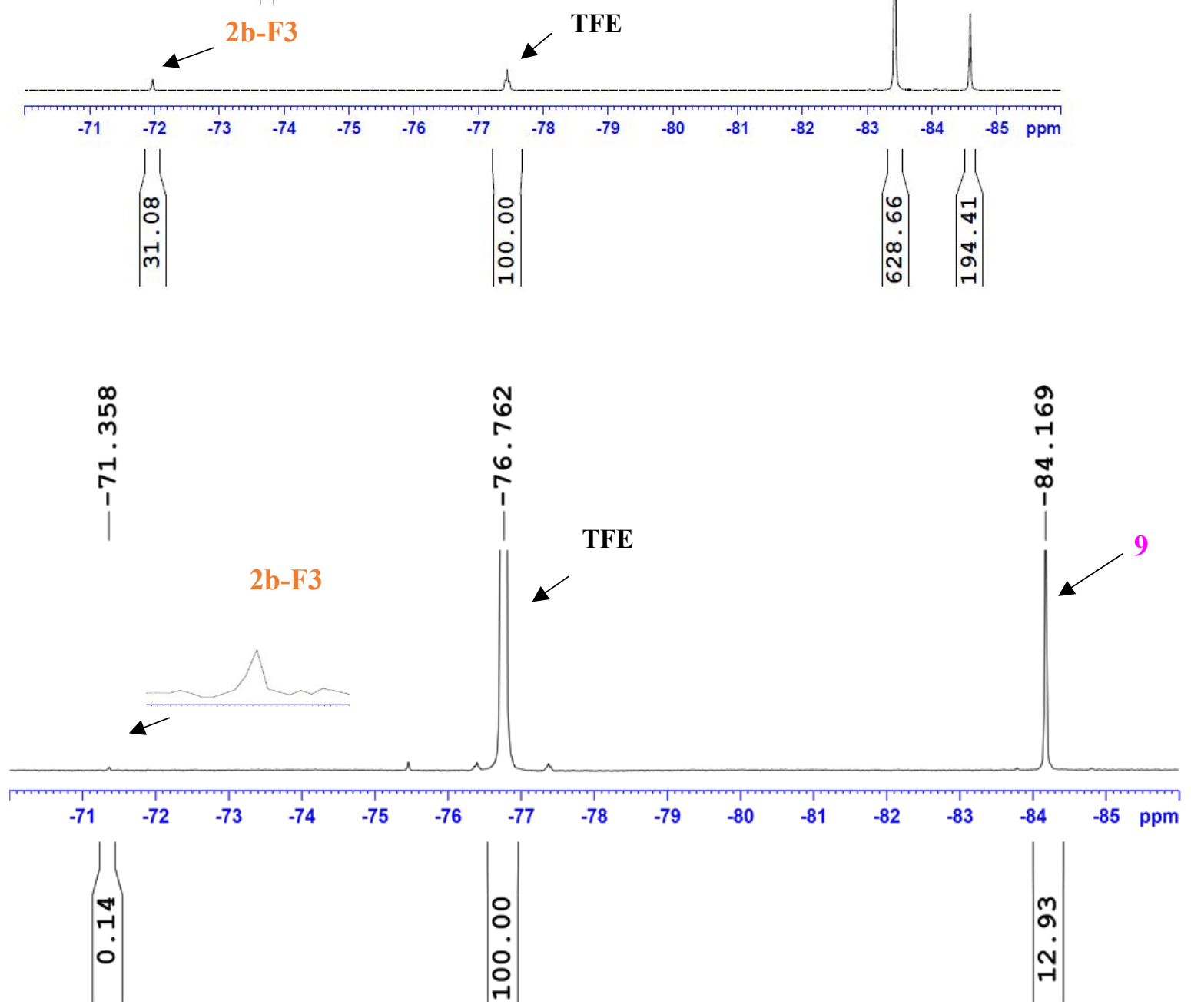

Figure S35. ${ }^{19} \mathrm{~F}$ NMR spectra of $\mathbf{2 b - F 3}$ in octanol (top) and $\mathrm{H}_{2} \mathrm{O}$ (down) after the separation of the phases for $\log P_{\mathrm{o} / \mathrm{w}}$ determination. 


\section{DFT Study (figures and calculations)}
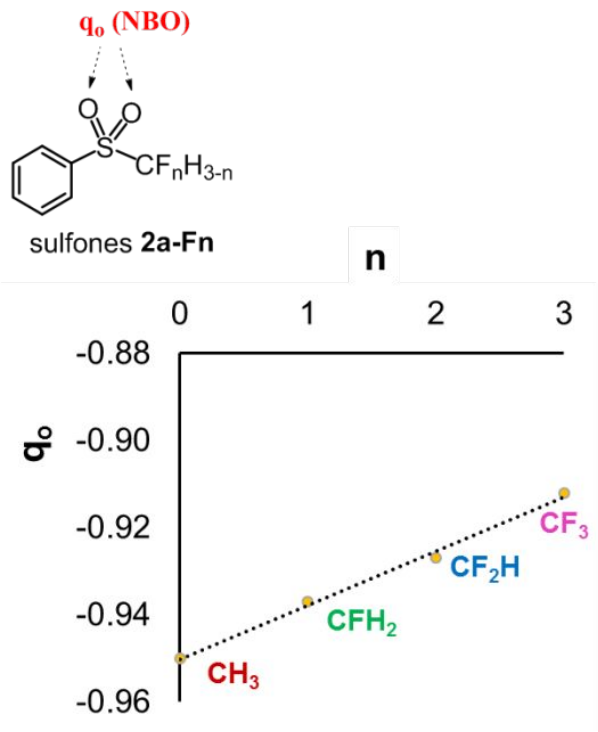
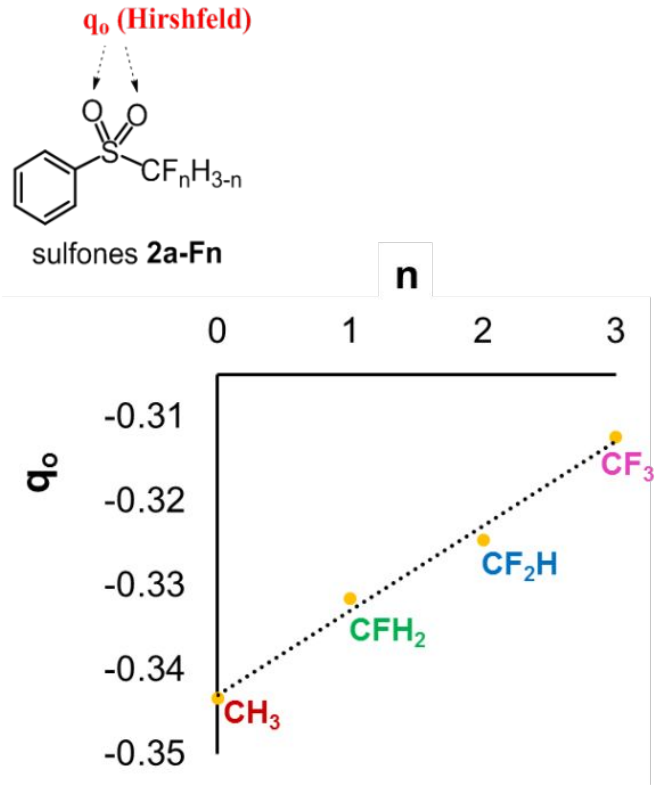

Figure S36. Oxygens average charges by NBO (left) and Hirshfeld (right) analyses for Sulfones 2a-Fn.

\section{2b-F Rotamer Energy Scan}

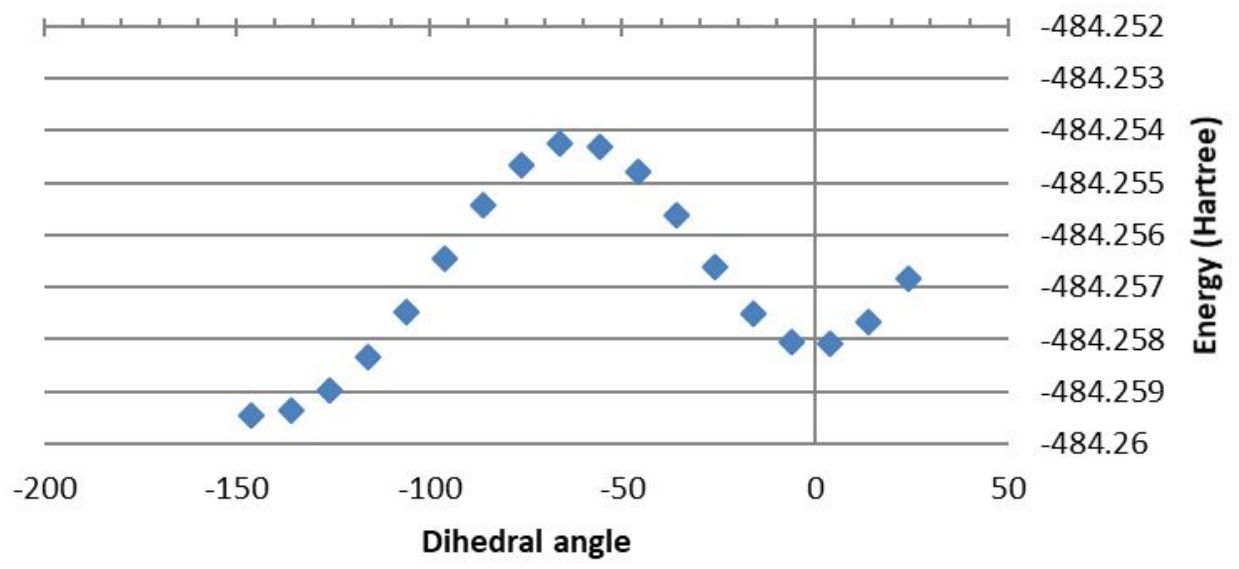

Figure S37. Analysis of rotamer energy by F-C-C-O dihedral angle. The calculation was performed at the B3LYP $\backslash 6-311++\mathrm{G}(\mathrm{d}, \mathrm{p})$ level of theory, with a $10^{\circ}$ increment per step. 

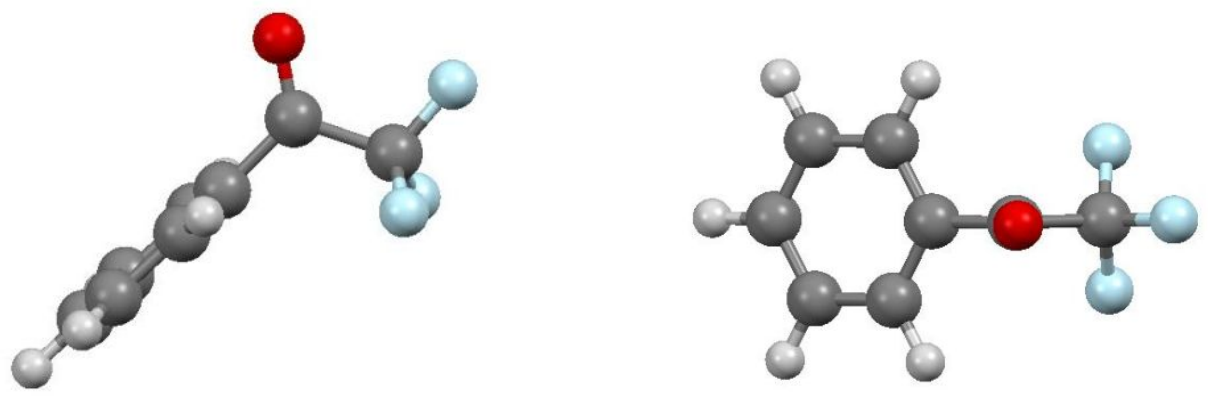

Figure S38. Geometry of a "frozen" carbonyl perpendicular to the phenyl ring plane (two views). Optimized at B3LYP/6-311++g(d,p) level of theory.
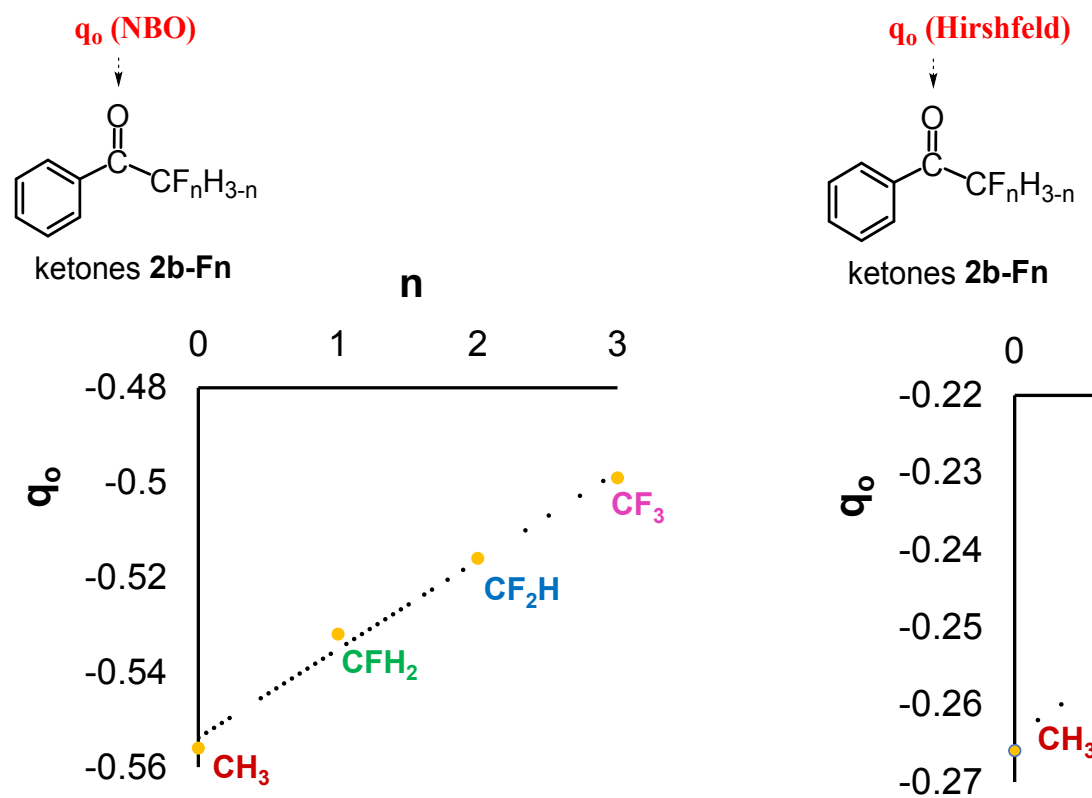

ketones 2b-Fn

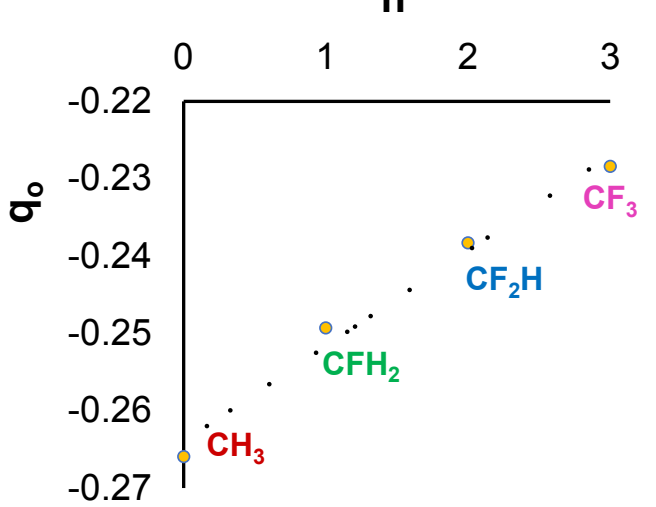

Figure S39. Oxygens average charges by NBO (left) and Hirshfeld (right) analyses for ketones 2b-Fn. 


\section{DFT calculations}

All calculations were performed at the m062x/6-311++g(d,p) level of theory. For all species, following optimization, frequency analysis (at the same base and functional) were performed, and all frequencies were verified to be positive. Unless otherwise noted, all 4 terms of convergence were found to be positive. In cases where this is not the case, the predicted change in energy is provided. Where the relative energy between two conformers was reported, it was based on the difference of energies of the sum of electronic and zero point energies (detailed here for the relevant species/conformers). Hirshfeld and NBO charge distribution analyses were performed on the optimized geometry of the species, at the same level of theory.

For each species, the following data is provided herein: atomic coordinates after optimization, charge distribution analysis by NBO and charge distribution analysis by Hirshfeld. For conformers compared to each other in energy, the sum of the electronic and zero-point energies from the frequency analysis is also reported.

\section{$\underline{\mathbf{2 a}}$}

\begin{tabular}{|c|c|c|c|c|c|}
\hline Center & Atomic & & tomic & Coordinate & es (Angstroms) \\
\hline Number & Numbe & & Type & $X$ & $\mathrm{Z}$ \\
\hline 1 & 6 & 0 & 2.377779 & -1.208957 & 0.036960 \\
\hline 2 & 6 & 0 & 0.990404 & -1.216070 & -0.054114 \\
\hline 3 & 6 & 0 & 0.319273 & 0.000002 & -0.092438 \\
\hline 4 & 6 & 0 & 0.990406 & 1.216071 & -0.054109 \\
\hline 5 & 6 & 0 & 2.377781 & 1.208955 & 0.036964 \\
\hline 6 & 6 & 0 & 3.066324 & -0.000001 & 0.085111 \\
\hline 7 & 1 & 0 & 2.921289 & -2.145542 & 0.060175 \\
\hline 8 & 1 & 0 & 0.429686 & -2.141502 & -0.119358 \\
\hline 9 & 1 & 0 & 0.429689 & 2.141504 & -0.119351 \\
\hline 10 & 1 & 0 & 2.921293 & 2.145539 & 0.060181 \\
\hline 11 & 1 & 0 & 4.147808 & -0.000003 & 0.152315 \\
\hline 12 & 16 & 0 & -1.465810 & 0.000002 & -0.170422 \\
\hline 13 & 8 & 0 & -1.888393 & 1.268290 & -0.746747 \\
\hline 14 & 8 & 0 & -1.888394 & -1.268280 & -0.746760 \\
\hline
\end{tabular}




$\begin{array}{llllll}15 & 6 & 0 & -1.955635 & -0.000010 & 1.550043 \\ 16 & 1 & 0 & -1.567503 & 0.901648 & 2.020513 \\ 17 & 1 & 0 & -3.045482 & -0.000010 & 1.549325 \\ 18 & 1 & 0 & -1.567507 & -0.901680 & 2.020493\end{array}$

Summary of Natural Population Analysis:

\begin{tabular}{|c|c|c|c|c|c|c|}
\hline Ator & & Charge & Core & Valence & Rydberg & Total \\
\hline $\mathrm{C}$ & 1 & -0.20017 & 1.99920 & 4.18362 & 0.01735 & 6.20017 \\
\hline $\mathrm{C}$ & 2 & -0.19455 & 1.99908 & 4.17581 & 0.01966 & 6.19455 \\
\hline $\mathrm{C}$ & 3 & -0.29189 & 1.99882 & 4.26441 & 0.02866 & 6.29189 \\
\hline $\mathrm{C}$ & 4 & -0.19455 & 1.99908 & 4.17581 & 0.01966 & 6.19455 \\
\hline $\mathrm{C}$ & 5 & -0.20017 & 1.99920 & 4.18362 & 0.01735 & 6.20017 \\
\hline $\mathrm{C}$ & 6 & -0.18299 & 1.99921 & 4.16687 & 0.01691 & 6.18299 \\
\hline $\mathrm{H}$ & 7 & 0.21842 & 0.00000 & 0.78014 & 0.00144 & 0.78158 \\
\hline $\mathrm{H}$ & 8 & 0.24289 & 0.00000 & 0.75514 & 0.00197 & 0.75711 \\
\hline $\mathrm{H}$ & 9 & 0.24289 & 0.00000 & 0.75514 & 0.00197 & 0.75711 \\
\hline $\mathrm{H}$ & 10 & 0.21842 & 0.00000 & 0.78014 & 0.00144 & 0.78158 \\
\hline $\mathrm{H}$ & 11 & 0.21603 & 0.00000 & 0.78261 & 0.00136 & 0.78397 \\
\hline $\mathrm{S}$ & 12 & 2.09816 & 9.99848 & 3.70123 & 0.20214 & 13.90184 \\
\hline $\mathrm{O}$ & 13 & -0.94554 & 1.99982 & 6.93299 & 0.01272 & 8.94554 \\
\hline $\mathrm{O}$ & 14 & -0.94554 & 1.99982 & 6.93299 & 0.01272 & 8.94554 \\
\hline $\mathrm{C}$ & 15 & -0.79687 & 1.99929 & 4.78501 & 0.01257 & 6.79687 \\
\hline $\mathrm{H}$ & 16 & 0.23435 & 0.00000 & 0.76388 & 0.00177 & 0.76565 \\
\hline $\mathrm{H}$ & 17 & 0.24679 & 0.00000 & 0.75116 & 0.00205 & 0.75321 \\
\hline $\mathrm{H}$ & 18 & 0.23435 & 0.00000 & 0.76388 & 0.00177 & 0.76565 \\
\hline
\end{tabular}




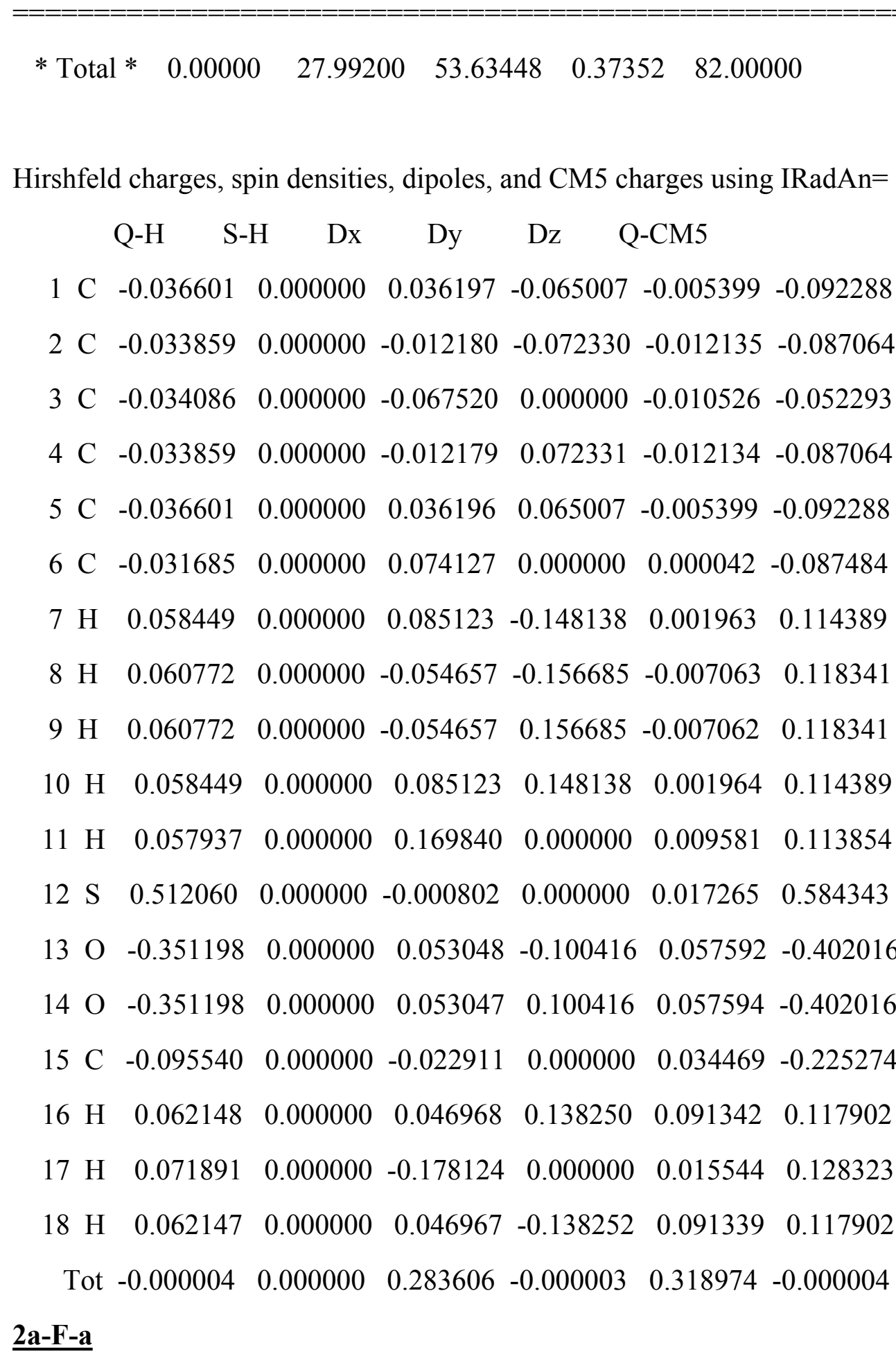

$4:$ 


\begin{tabular}{|c|c|c|c|c|c|}
\hline 1 & 6 & 0 & -2.584531 & 0.983837 & 0.615926 \\
\hline 2 & 6 & 0 & -1.217371 & 1.174153 & 0.453843 \\
\hline 3 & 6 & 0 & -0.482060 & 0.186698 & -0.191867 \\
\hline 4 & 6 & 0 & -1.066224 & -0.975173 & -0.682588 \\
\hline 5 & 6 & 0 & -2.433867 & -1.151761 & -0.510687 \\
\hline 6 & 6 & 0 & -3.187036 & -0.176597 & 0.137426 \\
\hline 7 & 1 & 0 & -3.179252 & 1.743635 & 1.107788 \\
\hline 8 & 1 & 0 & -0.723787 & 2.076250 & 0.796767 \\
\hline 9 & 1 & 0 & -0.456288 & -1.710064 & -1.194133 \\
\hline 10 & 1 & 0 & -2.911652 & -2.047650 & -0.887358 \\
\hline 11 & 1 & 0 & -4.253434 & -0.319880 & 0.266255 \\
\hline 12 & 16 & 0 & 1.276121 & 0.398513 & -0.360458 \\
\hline 13 & 8 & 0 & 1.728180 & -0.371977 & -1.504511 \\
\hline 14 & 8 & 0 & 1.603385 & 1.807841 & -0.201144 \\
\hline 15 & 6 & 0 & 1.935783 & -0.392676 & 1.136235 \\
\hline 16 & 1 & 0 & 1.530783 & 0.136711 & 1.999556 \\
\hline 17 & 1 & 0 & 3.021283 & -0.299388 & 1.066446 \\
\hline 18 & 9 & 0 & 1.568192 & -1.703736 & 1.167503 \\
\hline
\end{tabular}

Sum of electronic and zero-point Energies $=\quad-919.159908$

Summary of Natural Population Analysis:

$\begin{array}{crrrrrr}\text { Atom No } & \text { Charge } & \text { Core } & \text { Valence } & \text { Rydberg } & \text { Total } \\ \text { C } & 1 & -0.20179 & 1.99920 & 4.18515 & 0.01743 & 6.20179 \\ \text { C } & 2 & -0.19216 & 1.99908 & 4.17318 & 0.01990 & 6.19216 \\ \text { C } & 3 & -0.30288 & 1.99882 & 4.27582 & 0.02824 & 6.30288\end{array}$




$\begin{array}{ccccccc}\mathrm{C} & 4 & -0.17875 & 1.99908 & 4.15989 & 0.01978 & 6.17875 \\ \mathrm{C} & 5 & -0.19984 & 1.99920 & 4.18333 & 0.01731 & 6.19984 \\ \mathrm{C} & 6 & -0.17661 & 1.99921 & 4.16058 & 0.01681 & 6.17661 \\ \mathrm{H} & 7 & 0.21946 & 0.00000 & 0.77909 & 0.00145 & 0.78054 \\ \mathrm{H} & 8 & 0.24311 & 0.00000 & 0.75488 & 0.00201 & 0.75689 \\ \mathrm{H} & 9 & 0.24655 & 0.00000 & 0.75154 & 0.00191 & 0.75345 \\ \mathrm{H} & 10 & 0.22017 & 0.00000 & 0.77839 & 0.00144 & 0.77983 \\ \mathrm{H} & 11 & 0.21692 & 0.00000 & 0.78172 & 0.00136 & 0.78308 \\ \mathrm{~S} & 12 & 2.05459 & 9.99846 & 3.74145 & 0.20549 & 13.94541 \\ \mathrm{O} & 13 & -0.92432 & 1.99982 & 6.91136 & 0.01314 & 8.92432 \\ \mathrm{O} & 14 & -0.94023 & 1.99983 & 6.92776 & 0.01264 & 8.94023 \\ \mathrm{C} & 15 & -0.11321 & 1.99916 & 4.08211 & 0.03193 & 6.11321 \\ \mathrm{H} & 16 & 0.19047 & 0.00000 & 0.80733 & 0.00220 & 0.80953 \\ \mathrm{H} & 17 & 0.20093 & 0.00000 & 0.79680 & 0.00227 & 0.79907 \\ \mathrm{~F} & 18 & -0.36244 & 1.99993 & 7.35258 & 0.00993 & 9.36244\end{array}$

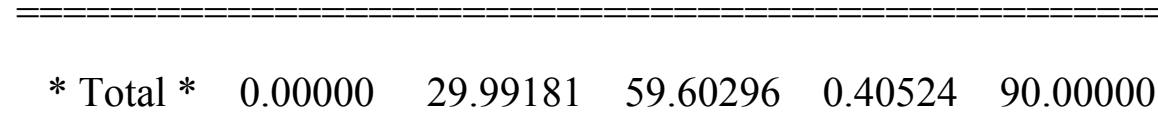

Hirshfeld charges, spin densities, dipoles, and CM5 charges using IRadAn $=\quad 4$ :

Q-H S-H Dx Dy Dz Q-CM5

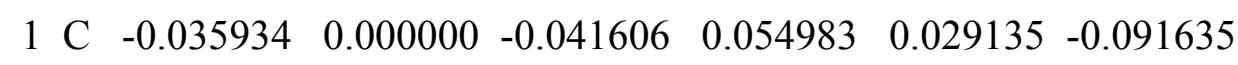

$\begin{array}{llllllll}2 & \mathrm{C} & -0.033319 & 0.000000 & 0.005441 & 0.070420 & 0.023058 & -0.086535\end{array}$

$\begin{array}{llllllll}3 & \mathrm{C} & -0.035438 & 0.000000 & 0.062595 & 0.011599 & -0.014035 & -0.053681\end{array}$

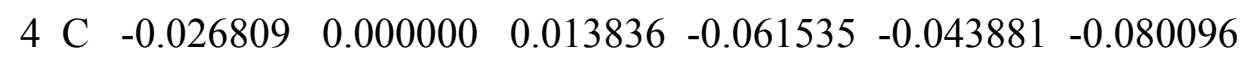

$\begin{array}{llllllll}5 & \mathrm{C} & -0.033481 & 0.000000 & -0.031855 & -0.062384 & -0.029295 & -0.089188\end{array}$

$\begin{array}{llllllll}6 & C & -0.027765 & 0.000000 & -0.074364 & -0.009345 & 0.006581 & -0.083541\end{array}$

$\begin{array}{llllllll}7 \mathrm{H} & 0.059350 & 0.000000 & -0.093921 & 0.121144 & 0.076870 & 0.115303\end{array}$

$\begin{array}{llllllll}8 \mathrm{H} & 0.060814 & 0.000000 & 0.042564 & 0.147695 & 0.063529 & 0.118411\end{array}$ 

$\begin{array}{llllllll}9 \mathrm{H} & 0.064464 & 0.000000 & 0.063622 & -0.131897 & -0.083445 & 0.121574\end{array}$
$\begin{array}{llllllll}10 & \mathrm{H} & 0.060335 & 0.000000 & -0.074694 & -0.142397 & -0.060661 & 0.116303\end{array}$

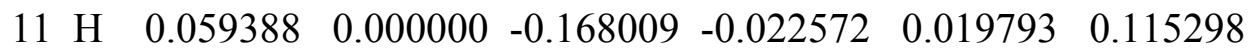
$\begin{array}{llllllll}12 & \mathrm{~S} & 0.502716 & 0.000000 & -0.004245 & 0.012792 & -0.007714 & 0.582785\end{array}$
$\begin{array}{llllllll}13 & \mathrm{O} & -0.335598 & 0.000000 & -0.049793 & 0.050053 & 0.088995 & -0.384575\end{array}$
$\begin{array}{llllllll}14 & \mathrm{O} & -0.343789 & 0.000000 & -0.043754 & -0.114631 & -0.002344 & -0.394729\end{array}$
$\begin{array}{llllllll}15 & \mathrm{C} & 0.030635 & 0.000000 & 0.015801 & -0.066964 & 0.035228 & -0.069229\end{array}$
$\begin{array}{llllllll}16 & \mathrm{H} & 0.066032 & 0.000000 & -0.045036 & 0.073590 & 0.150300 & 0.117013\end{array}$
$\begin{array}{llllllll}17 & \mathrm{H} & 0.075562 & 0.000000 & 0.179079 & 0.009974 & 0.004994 & 0.127038\end{array}$
$\begin{array}{llllllll}18 & \mathrm{~F} & -0.107221 & 0.000000 & 0.037872 & 0.115433 & -0.000578 & -0.080574\end{array}$
$\begin{array}{lllllll}\text { Tot } & -0.000057 & 0.000000 & -0.206469 & 0.055960 & 0.256529 & -0.000057\end{array}$

\section{$\underline{\text { 2a-F-g }}$}

\begin{tabular}{|c|c|c|c|c|}
\hline \multirow{2}{*}{$\begin{array}{l}\text { Center } \\
\text { Number }\end{array}$} & Atomic & Atomic & \multicolumn{2}{|c|}{ Coordinates (Angstroms) } \\
\hline & Numbe & Type & $X$ & Z \\
\hline 1 & 6 & 2.651244 & -1.209652 & 0.212814 \\
\hline 2 & 6 & 1.287921 & -1.217557 & -0.058812 \\
\hline 3 & 6 & 0.627898 & -0.000028 & -0.185564 \\
\hline 4 & 6 & 1.287873 & 1.217549 & -0.059017 \\
\hline 5 & 6 & 2.651196 & 1.209743 & 0.212613 \\
\hline 6 & 6 & 3.326670 & 0.000071 & 0.350794 \\
\hline 7 & 1 & 3.186922 & -2.146175 & 0.307379 \\
\hline 8 & 1 & 0.740961 & -2.143232 & -0.197280 \\
\hline 9 & 1 & 0.740870 & 2.143178 & -0.197624 \\
\hline 10 & 1 & 3.186834 & 2.146304 & 0.307028 \\
\hline 11 & 1 & 4.389933 & 0.000109 & 0.559543 \\
\hline 12 & 16 & -1.128353 & -0.000091 & $1-0.486022$ \\
\hline
\end{tabular}




$\begin{array}{llllll}13 & 8 & 0 & -1.496282 & 1.269611 & -1.088380 \\ 14 & 8 & 0 & -1.496277 & -1.270020 & -1.087903 \\ 15 & 6 & 0 & -1.750499 & 0.000220 & 1.231614 \\ 16 & 1 & 0 & -1.384985 & 0.908569 & 1.714039 \\ 17 & 1 & 0 & -1.384978 & -0.907956 & 1.714361 \\ 18 & 9 & 0 & -3.108360 & 0.000207 & 1.194722\end{array}$

Sum of electronic and zero-point Energies $=\quad-919.155484$

Summary of Natural Population Analysis:

\begin{tabular}{|c|c|c|c|c|c|c|}
\hline Aton & $\mathrm{m} \mathrm{I}$ & Charge & Core & Valence & Rydberg & Total \\
\hline $\mathrm{C}$ & 1 & -0.19965 & 1.99920 & 4.18304 & 0.01741 & 6.19965 \\
\hline $\mathrm{C}$ & 2 & -0.19147 & 1.99908 & 4.17217 & 0.02022 & 6.19147 \\
\hline $\mathrm{C}$ & 3 & -0.30949 & 1.99882 & 4.28199 & 0.02869 & 6.30949 \\
\hline $\mathrm{C}$ & 4 & -0.19147 & 1.99908 & 4.17217 & 0.02022 & 6.19147 \\
\hline $\mathrm{C}$ & 5 & -0.19966 & 1.99920 & 4.18304 & 0.01741 & 6.19966 \\
\hline $\mathrm{C}$ & 6 & -0.17644 & 1.99921 & 4.16044 & 0.01679 & 6.17644 \\
\hline $\mathrm{H}$ & 7 & 0.22067 & 0.00000 & 0.77789 & 0.00143 & 0.77933 \\
\hline $\mathrm{H}$ & 8 & 0.24447 & 0.00000 & 0.75351 & 0.00202 & 0.75553 \\
\hline $\mathrm{H}$ & 9 & 0.24446 & 0.00000 & 0.75352 & 0.00202 & 0.75554 \\
\hline $\mathrm{H}$ & 10 & 0.22067 & 0.00000 & 0.77789 & 0.00143 & 0.77933 \\
\hline $\mathrm{H}$ & 11 & 0.21796 & 0.00000 & 0.78069 & 0.00134 & 0.78204 \\
\hline $\mathrm{S}$ & 12 & 2.05603 & 9.99848 & 3.74115 & 0.20434 & 13.94397 \\
\hline $\mathrm{O}$ & 13 & -0.92820 & 1.99982 & 6.91536 & 0.01302 & 8.92820 \\
\hline $\mathrm{O}$ & 14 & -0.92820 & 1.99982 & 6.91536 & 0.01302 & 8.92820 \\
\hline $\mathrm{C}$ & 15 & -0.09858 & 1.99916 & 4.06772 & 0.03170 & 6.09858 \\
\hline
\end{tabular}




$\begin{array}{ccccccc}\mathrm{H} & 16 & 0.18753 & 0.00000 & 0.81034 & 0.00213 & 0.81247 \\ \mathrm{H} & 17 & 0.18753 & 0.00000 & 0.81034 & 0.00213 & 0.81247 \\ \mathrm{~F} & 18 & -0.35619 & 1.99993 & 7.34406 & 0.01219 & 9.35619\end{array}$

* Total * $\quad 0.00000 \quad 29.99182 \quad 59.60066 \quad 0.40752 \quad 90.00000$

Hirshfeld charges, spin densities, dipoles, and CM5 charges using IRadAn $=\quad 4$ : Q-H S-H Dx Dy $\quad$ Dz Q-CM5

$\begin{array}{llllllll}1 & C & -0.034180 & 0.000000 & -0.038459 & 0.065949 & -0.001013 & -0.089884\end{array}$

$\begin{array}{llllllll}2 & \mathrm{C} & -0.031621 & 0.000000 & 0.009645 & 0.073826 & -0.016423 & -0.084894\end{array}$

$\begin{array}{llllllll}3 \mathrm{C} & -0.036470 & 0.000000 & 0.062771 & 0.000003 & -0.022345 & -0.054931\end{array}$

$\begin{array}{llllllll}4 & C & -0.031622 & 0.000000 & 0.009648 & -0.073823 & -0.016438 & -0.084895\end{array}$

$\begin{array}{llllllll}5 & \mathrm{C} & -0.034181 & 0.000000 & -0.038457 & -0.065950 & -0.001025 & -0.089884\end{array}$

$\begin{array}{lllllll}6 \text { C } & -0.027084 & 0.000000 & -0.075570 & -0.000002 & 0.009893 & -0.082875\end{array}$

$\begin{array}{llllllll}7 \mathrm{H} & 0.060542 & 0.000000 & -0.084887 & 0.149217 & 0.013225 & 0.116490\end{array}$

$\begin{array}{llllllll}8 \mathrm{H} & 0.062600 & 0.000000 & 0.053299 & 0.158173 & -0.015564 & 0.120090\end{array}$

$\begin{array}{llllllll}9 \mathrm{H} & 0.062600 & 0.000000 & 0.053311 & -0.158168 & -0.015588 & 0.120089\end{array}$

$\begin{array}{llllllll}10 & \mathrm{H} & 0.060542 & 0.000000 & -0.084881 & -0.149222 & 0.013201 & 0.116490\end{array}$

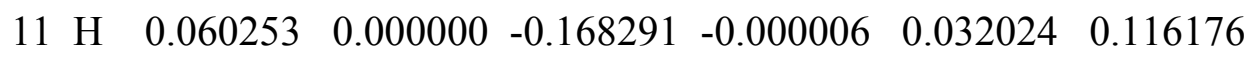

$\begin{array}{llllllll}12 & \mathrm{~S} & 0.503390 & 0.000000 & -0.011832 & 0.000003 & -0.007132 & 0.584281\end{array}$

$\begin{array}{llllllll}13 & \mathrm{O} & -0.337647 & 0.000000 & -0.043833 & 0.091898 & 0.052246 & -0.386852\end{array}$

$\begin{array}{llllllll}14 & \mathrm{O} & -0.337645 & 0.000000 & -0.043838 & -0.091915 & 0.052212 & -0.386851\end{array}$

$\begin{array}{llllllll}15 & \mathrm{C} & 0.030216 & 0.000000 & 0.073994 & -0.000007 & 0.036366 & -0.070203\end{array}$

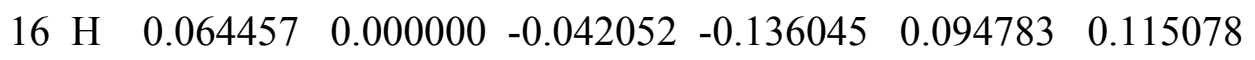

$\begin{array}{llllllll}17 & \mathrm{H} & 0.064458 & 0.000000 & -0.042054 & 0.136012 & 0.094832 & 0.115079\end{array}$

$\begin{array}{llllllll}18 & \text { F } & -0.098415 & 0.000000 & -0.119065 & 0.000000 & 0.000210 & -0.072314\end{array}$

$\begin{array}{lllllll}\text { Tot } & 0.000192 & 0.000000 & -0.530551 & -0.000057 & 0.303465 & 0.000192\end{array}$

\section{$\underline{\text { aa-F2-ag }}$}




\begin{tabular}{|c|c|c|c|c|c|}
\hline Center & Atomic & & tomic & Coordinates & es (Angstrom \\
\hline Number & Numbe & & Type & $\mathrm{X}$ & Z \\
\hline 1 & 6 & 0 & -2.680417 & -1.178254 & 0.482661 \\
\hline 2 & 6 & 0 & -1.338181 & -0.929751 & 0.739067 \\
\hline 3 & 6 & 0 & -0.764494 & 0.219429 & 0.205029 \\
\hline 4 & 6 & 0 & -1.485823 & 1.125034 & -0.564783 \\
\hline 5 & 6 & 0 & -2.829317 & 0.863431 & -0.808803 \\
\hline 6 & 6 & 0 & -3.419674 & -0.284871 & -0.289007 \\
\hline 7 & 1 & 0 & -3.150413 & -2.065020 & 0.889262 \\
\hline 8 & 1 & 0 & -0.740420 & -1.598281 & 1.347310 \\
\hline 9 & 1 & 0 & -1.002659 & 2.020656 & -0.937717 \\
\hline 10 & 1 & 0 & -3.415227 & 1.558311 & -1.397547 \\
\hline 11 & 1 & 0 & -4.467147 & -0.483540 & -0.482931 \\
\hline 12 & 16 & 0 & 0.962019 & 0.526114 & 0.489308 \\
\hline 13 & 8 & 0 & 1.250256 & 1.917654 & 0.192959 \\
\hline 14 & 8 & 0 & 1.364248 & -0.110113 & 1.727067 \\
\hline 15 & 6 & 0 & 1.733931 & -0.430882 & -0.896220 \\
\hline 16 & 9 & 0 & 1.356834 & -1.714957 & -0.798525 \\
\hline 17 & 9 & 0 & 3.059763 & -0.352513 & -0.752754 \\
\hline 18 & 1 & 0 & 1.421999 & -0.007866 & -1.853662 \\
\hline
\end{tabular}

Sum of electronic and zero-point Energies $=\quad-1018.407983$

Summary of Natural Population Analysis:

\begin{tabular}{|c|c|c|c|c|}
\hline Atom No & Charge & Core & Valence & Rydberg \\
\hline
\end{tabular}




$\begin{array}{lrrrrrr}\text { C } & 2 & -0.17667 & 1.99908 & 4.15772 & 0.01987 & 6.17667 \\ \text { C } & 3 & -0.31436 & 1.99882 & 4.28718 & 0.02836 & 6.31436 \\ \text { C } & 4 & -0.18981 & 1.99908 & 4.17039 & 0.02033 & 6.18981 \\ \text { C } & 5 & -0.20128 & 1.99920 & 4.18458 & 0.01749 & 6.20128 \\ \text { C } & 6 & -0.17226 & 1.99921 & 4.15626 & 0.01678 & 6.17226 \\ \text { H } & 7 & 0.22175 & 0.00000 & 0.77680 & 0.00145 & 0.77825 \\ \text { H } & 8 & 0.24670 & 0.00000 & 0.75138 & 0.00192 & 0.75330 \\ \text { H } & 9 & 0.24519 & 0.00000 & 0.75276 & 0.00205 & 0.75481 \\ \text { H } & 10 & 0.22109 & 0.00000 & 0.77746 & 0.00145 & 0.77891 \\ \text { H } & 11 & 0.21826 & 0.00000 & 0.78039 & 0.00135 & 0.78174 \\ \text { S } & 12 & 2.03018 & 9.99844 & 3.76185 & 0.20952 & 13.96982 \\ \text { O } & 13 & -0.92603 & 1.99982 & 6.91322 & 0.01299 & 8.92603 \\ \text { O } & 14 & -0.90956 & 1.99982 & 6.89637 & 0.01337 & 8.90956 \\ \text { C } & 15 & 0.44409 & 1.99943 & 3.50215 & 0.05432 & 5.55591 \\ \text { F } & 16 & -0.35370 & 1.99992 & 7.34469 & 0.00909 & 9.35370 \\ \text { F } & 17 & -0.34139 & 1.99992 & 7.33289 & 0.00857 & 9.34139 \\ \text { H } & 18 & 0.15691 & 0.00000 & 0.84037 & 0.00272 & 0.84309\end{array}$

* Total * $\quad 0.00000 \quad 31.99196 \quad 65.56904 \quad 0.43900 \quad 98.00000$ Hirshfeld charges, spin densities, dipoles, and CM5 charges using IRadAn $=\quad 4$ :

Q-H S-H Dx Dy $\quad$ Dz $\quad$ Q-CM5

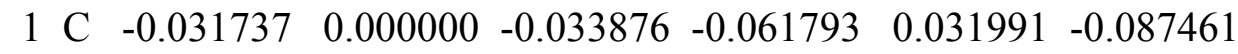

$\begin{array}{llllllll}2 \mathrm{C} & -0.026245 & 0.000000 & 0.012339 & -0.057124 & 0.051818 & -0.079569\end{array}$

$\begin{array}{llllllll}3 & \mathrm{C} & -0.038389 & 0.000000 & 0.057637 & 0.016902 & 0.021284 & -0.056656\end{array}$

$\begin{array}{lllllll}4 \mathrm{C} & -0.031932 & 0.000000 & 0.001856 & 0.071215 & -0.026772 & -0.085121\end{array}$

$\begin{array}{llllllll}5 & \mathrm{C} & -0.034264 & 0.000000 & -0.042068 & 0.052385 & -0.036033 & -0.089972\end{array}$

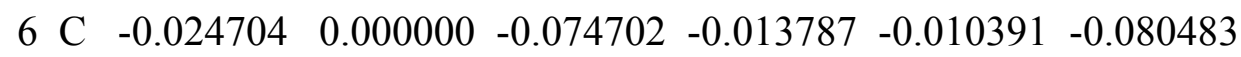

$\begin{array}{llllllll}7 \mathrm{H} & 0.061851 & 0.000000 & -0.074157 & -0.141507 & 0.065843 & 0.117828\end{array}$ 

$\begin{array}{llllllll}8 \mathrm{H} & 0.065564 & 0.000000 & 0.062754 & -0.122702 & 0.099046 & 0.122371\end{array}$
$\begin{array}{llllllll}9 \mathrm{H} & 0.061942 & 0.000000 & 0.040571 & 0.146012 & -0.071112 & 0.119717\end{array}$
$\begin{array}{llllllll}10 & \mathrm{H} & 0.060878 & 0.000000 & -0.093284 & 0.111582 & -0.092699 & 0.116838\end{array}$
$\begin{array}{llllllll}11 \mathrm{H} & 0.060990 & 0.000000 & -0.165916 & -0.031353 & -0.030148 & 0.116911\end{array}$
$\begin{array}{llllllll}12 & \mathrm{~S} & 0.494765 & 0.000000 & -0.023946 & 0.028250 & 0.022194 & 0.583069\end{array}$
$\begin{array}{llllllll}13 & \mathrm{O} & -0.332209 & 0.000000 & -0.039767 & -0.101572 & 0.015075 & -0.381656\end{array}$
$\begin{array}{llllllll}14 & \mathrm{O} & -0.324210 & 0.000000 & -0.042622 & 0.040000 & -0.084036 & -0.371525\end{array}$
$\begin{array}{llllllll}15 & \mathrm{C} & 0.135438 & 0.000000 & 0.054079 & -0.056480 & -0.022826 & 0.062372\end{array}$
$\begin{array}{llllllll}16 & \mathrm{~F} & -0.089772 & 0.000000 & 0.027756 & 0.094081 & -0.010921 & -0.075654\end{array}$
$\begin{array}{llllllll}17 & \mathrm{~F} & -0.079277 & 0.000000 & -0.095075 & 0.001350 & -0.011410 & -0.065989\end{array}$
$\begin{array}{llllllll}18 & \mathrm{H} & 0.071439 & 0.000000 & -0.034386 & 0.056059 & -0.164978 & 0.135108\end{array}$
$\begin{array}{lllllll}\text { Tot } & 0.000128 & 0.000000 & -0.462808 & 0.031519 & -0.254075 & 0.000128\end{array}$

\section{2a-F2-aa}

\begin{tabular}{|c|c|c|c|c|}
\hline \multirow{2}{*}{$\begin{array}{l}\text { Center } \\
\text { Number }\end{array}$} & Atomic & \multirow{2}{*}{$\begin{array}{l}\text { Atomic } \\
\text { Type }\end{array}$} & \multicolumn{2}{|c|}{ Coordinates (Angstroms) } \\
\hline & Number & & $\mathrm{X}$ & Z \\
\hline 1 & 6 & -2.622397 & 1.210199 & 0.144414 \\
\hline 2 & 6 & -1.275685 & 1.220059 & -0.196243 \\
\hline 3 & 6 & -0.628024 & 0.000570 & -0.361113 \\
\hline 4 & 6 & -1.275510 & -1.219447 & -0.199487 \\
\hline 5 & 6 & -2.622220 & -1.210687 & 0.141207 \\
\hline 6 & 6 & -3.289281 & -0.000519 & 0.312944 \\
\hline 7 & 1 & -3.151298 & 2.146273 & 0.273229 \\
\hline 8 & 1 & -0.731878 & 2.144816 & -0.347691 \\
\hline 9 & 1 & -0.731551 & -2.143716 & -0.353367 \\
\hline 10 & 1 & -3.150979 & -2.147180 & 0.267542 \\
\hline
\end{tabular}




$\begin{array}{lccrrr}11 & 1 & 0 & -4.340276 & -0.000951 & 0.577351 \\ 12 & 16 & 0 & 1.098640 & 0.001227 & -0.761967 \\ 13 & 8 & 0 & 1.459557 & -1.268239 & -1.364922 \\ 14 & 8 & 0 & 1.459555 & 1.272583 & -1.360914 \\ 15 & 6 & 0 & 1.900188 & -0.001448 & 0.901682 \\ 16 & 1 & 0 & 2.983209 & -0.001327 & 0.761478 \\ 17 & 9 & 0 & 1.503741 & -1.091971 & 1.573783 \\ 18 & 9 & 0 & 1.503948 & 1.087009 & 1.577240\end{array}$

Sum of electronic and zero-point Energies $=\quad-1018.411006$

Note: 3 out of 4 convergence criteria of the frequencies section were met. Predicted change in energy: -1.435813D-07.

Summary of Natural Population Analysis:

$\begin{array}{llllllll}\text { Atom No } & \text { Charge } & \text { Core } & \text { Valence } & \text { Rydberg } & \text { Total } \\ \text { C } & 1 & -0.20161 & 1.99920 & 4.18504 & 0.01737 & 6.20161 \\ \text { C } & 2 & -0.17651 & 1.99908 & 4.15756 & 0.01987 & 6.17651 \\ \text { C } & 3 & -0.31412 & 1.99882 & 4.28649 & 0.02880 & 6.31412 \\ \text { C } & 4 & -0.17651 & 1.99908 & 4.15756 & 0.01987 & 6.17651 \\ \text { C } & 5 & -0.20161 & 1.99920 & 4.18504 & 0.01737 & 6.20161 \\ \text { C } & 6 & -0.17064 & 1.99921 & 4.15470 & 0.01673 & 6.17064 \\ \text { H } & 7 & 0.22090 & 0.00000 & 0.77764 & 0.00146 & 0.77910 \\ \text { H } & 8 & 0.24598 & 0.00000 & 0.75203 & 0.00200 & 0.75402 \\ \text { H } & 9 & 0.24597 & 0.00000 & 0.75203 & 0.00200 & 0.75403 \\ \text { H } & 10 & 0.22090 & 0.00000 & 0.77764 & 0.00146 & 0.77910 \\ \text { H } & 11 & 0.21760 & 0.00000 & 0.78104 & 0.00136 & 0.78240 \\ \text { S } & 12 & 2.02911 & 9.99844 & 3.76206 & 0.21039 & 13.97089 \\ \text { O } & 13 & -0.92196 & 1.99982 & 6.90920 & 0.01293 & 8.92196 \\ & & & & & 540 & \end{array}$




$\begin{array}{lrrrrrr}\text { O } & 14 & -0.92195 & 1.99982 & 6.90920 & 0.01293 & 8.92195 \\ \text { C } & 15 & 0.43596 & 1.99944 & 3.51095 & 0.05365 & 5.56404 \\ \text { H } & 16 & 0.16709 & 0.00000 & 0.83006 & 0.00284 & 0.83291 \\ \text { F } & 17 & -0.34931 & 1.99992 & 7.34180 & 0.00759 & 9.34931 \\ \text { F } & 18 & -0.34931 & 1.99992 & 7.34179 & 0.00759 & 9.34931\end{array}$

\section{$\begin{array}{llllll}* \text { Total } & 0.00000 & 31.99196 & 65.57185 & 0.43619 & 98.00000\end{array}$}

Hirshfeld charges, spin densities, dipoles, and CM5 charges using IRadAn= $\quad 4$ :
$\begin{array}{llllll}\text { Q-H } & \text { S-H } & \text { Dx } & \text { Dy } & \text { Dz } & \text { Q-CM5 }\end{array}$
$\begin{array}{llllllll}1 & C & -0.033137 & 0.000000 & -0.036576 & 0.066153 & 0.007509 & -0.088857\end{array}$
$\begin{array}{llllllll}2 & \mathrm{C} & -0.026622 & 0.000000 & 0.007515 & 0.075898 & -0.009775 & -0.079900\end{array}$
$\begin{array}{llllllll}3 & \mathrm{C} & -0.037124 & 0.000000 & 0.056506 & 0.000038 & -0.023660 & -0.055384\end{array}$
$\begin{array}{llllllll}4 & \mathrm{C} & -0.026623 & 0.000000 & 0.007517 & -0.075868 & -0.009984 & -0.079901\end{array}$
$\begin{array}{llllllll}5 & \mathrm{C} & -0.033137 & 0.000000 & -0.036569 & -0.066178 & 0.007335 & -0.088857\end{array}$
$\begin{array}{llllllll}6 \text { C } & -0.024258 & 0.000000 & -0.073692 & -0.000030 & 0.018035 & -0.080018\end{array}$
$\begin{array}{llllllll}7 \mathrm{H} & 0.060939 & 0.000000 & -0.083319 & 0.149443 & 0.020082 & 0.116917\end{array}$
$\begin{array}{llllllll}8 \mathrm{H} & 0.064225 & 0.000000 & 0.051932 & 0.159713 & -0.016047 & 0.121402\end{array}$
$\begin{array}{llllllll}9 \mathrm{H} & 0.064224 & 0.000000 & 0.051960 & -0.159662 & -0.016470 & 0.121399\end{array}$
$\begin{array}{lllllll}10 \mathrm{H} & 0.060940 & 0.000000 & -0.083296 & -0.149508 & 0.019685 & 0.116917\end{array}$
$\begin{array}{llllllll}11 \mathrm{H} & 0.060576 & 0.000000 & -0.165934 & -0.000069 & 0.041736 & 0.116483\end{array}$
$\begin{array}{llllllll}12 & \mathrm{~S} & 0.494779 & 0.000000 & -0.009145 & 0.000073 & -0.039202 & 0.582667\end{array}$
$\begin{array}{lllllllll}13 & \mathrm{O} & -0.332258 & 0.000000 & -0.043451 & 0.087934 & 0.049177 & -0.380963\end{array}$
$\begin{array}{llllllll}14 & \mathrm{O} & -0.332248 & 0.000000 & -0.043460 & -0.088078 & 0.048902 & -0.380954\end{array}$
$\begin{array}{llllllll}15 & \mathrm{C} & 0.137150 & 0.000000 & 0.007085 & -0.000120 & 0.076381 & 0.064502\end{array}$
$\begin{array}{llllllll}16 & \mathrm{H} & 0.081178 & 0.000000 & 0.183945 & 0.000001 & -0.009690 & 0.126724\end{array}$
$\begin{array}{llllllll}17 & \mathrm{~F} & -0.089226 & 0.000000 & 0.038716 & 0.077031 & -0.049115 & -0.066011\end{array}$
$\begin{array}{llllllll}18 & \mathrm{~F} & -0.089228 & 0.000000 & 0.038710 & -0.076879 & -0.049354 & -0.066014\end{array}$ 
$\begin{array}{lllllll}\text { Tot } & 0.000152 & 0.000000 & -0.131555 & -0.000107 & 0.065541 & 0.000152\end{array}$

Hirshfeld charges with hydrogens summed into heavy atoms:

\section{2a-F3-aag}

\begin{tabular}{|c|c|c|c|c|c|}
\hline Center & Atomic & & omic & Coordinate & es (Angstroms) \\
\hline Number & Numbe & & Type & $X$ & Z \\
\hline 1 & 6 & 0 & 2.845547 & 1.210607 & -0.255030 \\
\hline 2 & 6 & 0 & 1.525753 & 1.220472 & 0.177630 \\
\hline 3 & 6 & 0 & 0.891290 & 0.000255 & 0.384187 \\
\hline 4 & 6 & 0 & 1.525635 & -1.220193 & 0.178506 \\
\hline 5 & 6 & 0 & 2.845374 & -1.210830 & -0.254195 \\
\hline 6 & 6 & 0 & 3.498683 & -0.000222 & -0.470769 \\
\hline 7 & 1 & 0 & 3.364457 & 2.146680 & -0.419210 \\
\hline 8 & 1 & 0 & 0.993330 & 2.145371 & 0.365073 \\
\hline 9 & 1 & 0 & 0.993047 & -2.144871 & 0.366518 \\
\hline 10 & 1 & 0 & 3.364120 & -2.147100 & -0.417784 \\
\hline 11 & 1 & 0 & 4.528467 & -0.000421 & -0.808040 \\
\hline 12 & 16 & 0 & -0.807135 & 0.000352 & 0.888611 \\
\hline 13 & 8 & 0 & -1.136870 & -1.270727 & 1.496258 \\
\hline 14 & 8 & 0 & -1.137071 & 1.271397 & 1.496176 \\
\hline 15 & 6 & 0 & -1.677039 & -0.000153 & -0.763668 \\
\hline 16 & 9 & 0 & -1.329117 & -1.076278 & -1.456271 \\
\hline 17 & 9 & 0 & -1.341221 & 1.083163 & -1.450853 \\
\hline 18 & 9 & 0 & -2.981794 & -0.008027 & -0.562183 \\
\hline
\end{tabular}


Note: 3 out of 4 convergence criteria of the frequencies section were met. Predicted change in energy: -2.099807D-08.

Summary of Natural Population Analysis:

\begin{tabular}{llllllll} 
Atom No & Charge & Core & Valence & Rydberg & Total \\
\hline C & 1 & -0.19994 & 1.99920 & 4.18333 & 0.01741 & 6.19994 \\
C & 2 & -0.17680 & 1.99908 & 4.15772 & 0.02000 & 6.17680 \\
C & 3 & -0.31930 & 1.99882 & 4.29221 & 0.02827 & 6.31930 \\
C & 4 & -0.17670 & 1.99908 & 4.15762 & 0.01999 & 6.17670 \\
C & 5 & -0.19993 & 1.99920 & 4.18332 & 0.01741 & 6.19993 \\
C & 6 & -0.16763 & 1.99921 & 4.15169 & 0.01673 & 6.16763 \\
H & 7 & 0.22239 & 0.00000 & 0.77615 & 0.00146 & 0.77761 \\
H & 8 & 0.24669 & 0.00000 & 0.75130 & 0.00201 & 0.75331 \\
H & 9 & 0.24669 & 0.00000 & 0.75130 & 0.00201 & 0.75331 \\
H & 10 & 0.22239 & 0.00000 & 0.77615 & 0.00146 & 0.77761 \\
H & 11 & 0.21897 & 0.00000 & 0.77967 & 0.00136 & 0.78103 \\
S & 12 & 2.02707 & 9.99835 & 3.75912 & 0.21546 & 13.97293 \\
O & 13 & -0.90719 & 1.99982 & 6.89434 & 0.01302 & 8.90719 \\
O & 14 & -0.90713 & 1.99982 & 6.89428 & 0.01302 & 8.90713 \\
C & 15 & 0.88865 & 1.99982 & 3.03434 & 0.07719 & 5.11135 \\
F & 16 & -0.34319 & 1.99991 & 7.33303 & 0.01025 & 9.34319 \\
F & 17 & -0.34307 & 1.99991 & 7.33291 & 0.01025 & 9.34307 \\
F & 18 & -0.33198 & 1.99991 & 7.32282 & 0.00925 & 9.33198
\end{tabular}

* Total * $\quad 0.00000 \quad 33.99214 \quad 71.53130 \quad 0.47657 \quad 106.00000$

Hirshfeld charges, spin densities, dipoles, and CM5 charges using IRadAn $=\quad 4$ :

Q-H S-H Dx $\quad$ Dy $\quad$ Dz $\quad$ Q-CM5 

$\begin{array}{llllllll}1 & C & -0.031352 & 0.000000 & 0.037320 & 0.067052 & -0.009593 & -0.087082\end{array}$
$\begin{array}{llllllll}2 & \mathrm{C} & -0.026368 & 0.000000 & -0.005796 & 0.077283 & 0.012277 & -0.079634\end{array}$
$\begin{array}{llllllll}3 & \mathrm{C} & -0.039290 & 0.000000 & -0.052358 & 0.000009 & 0.031415 & -0.057516\end{array}$

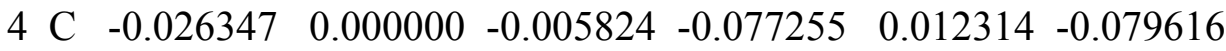
$\begin{array}{llllllll}5 & \mathrm{C} & -0.031340 & 0.000000 & 0.037339 & -0.067066 & -0.009583 & -0.087069\end{array}$

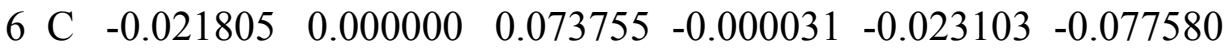
$\begin{array}{llllllll}7 & \mathrm{H} & 0.062421 & 0.000000 & 0.082380 & 0.150160 & -0.025737 & 0.118406\end{array}$
$\begin{array}{llllllll}8 & \mathrm{H} & 0.065335 & 0.000000 & -0.050905 & 0.160782 & 0.020259 & 0.122507\end{array}$
$\begin{array}{llllllll}9 & \mathrm{H} & 0.065319 & 0.000000 & -0.050896 & -0.160762 & 0.020336 & 0.122496\end{array}$
$\begin{array}{llllllll}10 & \mathrm{H} & 0.062426 & 0.000000 & 0.082349 & -0.150191 & -0.025646 & 0.118410\end{array}$
$\begin{array}{llllllll}11 & \mathrm{H} & 0.062093 & 0.000000 & 0.163483 & -0.000035 & -0.053442 & 0.118020\end{array}$
$\begin{array}{llllllll}12 & \mathrm{~S} & 0.493320 & 0.000000 & 0.030547 & 0.000057 & 0.055825 & 0.587587\end{array}$
$\begin{array}{llllllll}13 & \mathrm{O} & -0.319836 & 0.000000 & 0.038716 & 0.078982 & -0.040747 & -0.367410\end{array}$
$\begin{array}{llllllll}14 & \mathrm{O} & -0.319826 & 0.000000 & 0.038678 & -0.079002 & -0.040723 & -0.367392\end{array}$
$\begin{array}{llllllll}15 & \mathrm{C} & 0.223051 & 0.000000 & -0.042799 & -0.000020 & -0.064549 & 0.176247\end{array}$

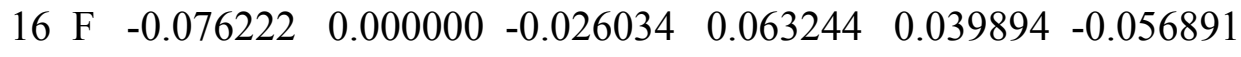
$\begin{array}{llllllll}17 & F & -0.076168 & 0.000000 & -0.025341 & -0.063696 & 0.039485 & -0.056847\end{array}$
$\begin{array}{llllllll}18 & \text { F } & -0.065259 & 0.000000 & 0.074963 & 0.000503 & -0.007176 & -0.046486\end{array}$
$\begin{array}{llllllll}\text { Tot } & 0.000151 & 0.000000 & 0.399577 & 0.000013 & -0.068494 & 0.000151\end{array}$

\section{$\underline{2 b}$}

\begin{tabular}{lccccc} 
Center & Atomic & Atomic & \multicolumn{3}{c}{ Coordinates (Angstroms) } \\
Number & Number & Type & $X$ & $Y$ & $Z$
\end{tabular}




\begin{tabular}{|c|c|c|c|c|c|}
\hline 1 & 6 & 0 & 1.811807 & 1.274978 & 0.000009 \\
\hline 2 & 6 & 0 & 0.423695 & 1.187531 & 0.000012 \\
\hline 3 & 6 & 0 & -0.201698 & -0.059961 & 0.000005 \\
\hline 4 & 6 & 0 & 0.576972 & -1.219770 & -0.000006 \\
\hline 5 & 6 & 0 & 1.961227 & -1.132420 & -0.000010 \\
\hline 6 & 6 & 0 & 2.580194 & 0.115933 & -0.000002 \\
\hline 7 & 1 & 0 & 2.292457 & 2.245845 & 0.000015 \\
\hline 8 & 1 & 0 & -0.165190 & 2.097216 & 0.000020 \\
\hline 9 & 1 & 0 & 0.071908 & -2.178331 & -0.000011 \\
\hline 10 & 1 & 0 & 2.561053 & -2.034808 & -0.000018 \\
\hline 11 & 1 & 0 & 3.661803 & 0.184365 & -0.000005 \\
\hline 12 & 6 & 0 & -1.696787 & -0.206656 & 0.000010 \\
\hline 13 & 8 & 0 & -2.206051 & -1.302358 & -0.000005 \\
\hline 14 & 6 & 0 & -2.543304 & 1.046799 & -0.000006 \\
\hline 15 & 1 & 0 & -2.327090 & 1.653543 & 0.882555 \\
\hline 16 & 1 & 0 & -2.327057 & 1.653547 & -0.882555 \\
\hline 17 & 1 & 0 & -3.592109 & 0.758888 & -0.000025 \\
\hline
\end{tabular}

Summary of Natural Population Analysis:

$\begin{array}{rrrrrrr}\text { Atom No } & \text { Charge } & \text { Core } & \text { Valence } & \text { Rydberg } & \text { Total } \\ \text { C } & 1 & -0.21087 & 1.99921 & 4.19417 & 0.01749 & 6.21087 \\ \text { C } & 2 & -0.18232 & 1.99914 & 4.16836 & 0.01481 & 6.18232 \\ \text { C } & 3 & -0.15978 & 1.99899 & 4.14324 & 0.01755 & 6.15978 \\ \text { C } & 4 & -0.15886 & 1.99913 & 4.14204 & 0.01769 & 6.15886 \\ \text { C } & 5 & -0.20728 & 1.99920 & 4.19125 & 0.01683 & 6.20728\end{array}$




$\begin{array}{ccccccc}\text { C } & 6 & -0.18482 & 1.99921 & 4.16859 & 0.01702 & 6.18482 \\ \mathrm{H} & 7 & 0.21282 & 0.00000 & 0.78567 & 0.00150 & 0.78718 \\ \mathrm{H} & 8 & 0.21252 & 0.00000 & 0.78592 & 0.00156 & 0.78748 \\ \mathrm{H} & 9 & 0.23569 & 0.00000 & 0.76238 & 0.00193 & 0.76431 \\ \mathrm{H} & 10 & 0.21381 & 0.00000 & 0.78476 & 0.00144 & 0.78619 \\ \mathrm{H} & 11 & 0.21211 & 0.00000 & 0.78651 & 0.00138 & 0.78789 \\ \mathrm{C} & 12 & 0.57337 & 1.99934 & 3.38776 & 0.03953 & 5.42663 \\ \mathrm{O} & 13 & -0.55549 & 1.99977 & 6.54128 & 0.01444 & 8.55549 \\ \mathrm{C} & 14 & -0.68174 & 1.99932 & 4.67449 & 0.00793 & 6.68174 \\ \mathrm{H} & 15 & 0.22360 & 0.00000 & 0.77486 & 0.00154 & 0.77640 \\ \mathrm{H} & 16 & 0.22360 & 0.00000 & 0.77486 & 0.00154 & 0.77640 \\ \mathrm{H} & 17 & 0.23364 & 0.00000 & 0.76450 & 0.00186 & 0.76636\end{array}$

* Total * $\quad 0.00000 \quad 17.99331 \quad 45.83067 \quad 0.17602 \quad 64.00000$

Hirshfeld charges, spin densities, dipoles, and CM5 charges using IRadAn $=\quad 4$ :

Q-H S-H Dx Dy $\quad$ Dz $\quad$ Q-CM5

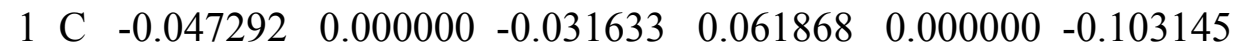

$\begin{array}{llllllll}2 & \mathrm{C} & -0.042353 & 0.000000 & 0.032503 & 0.054889 & 0.000000 & -0.095706\end{array}$

$\begin{array}{llllllll}3 & \mathrm{C} & -0.023423 & 0.000000 & 0.063404 & 0.000745 & 0.000000 & -0.029953\end{array}$

$\begin{array}{llllllll}4 & C & -0.031567 & 0.000000 & 0.023320 & -0.060794 & 0.000001 & -0.083313\end{array}$

$\begin{array}{llllllll}5 & \mathrm{C} & -0.043117 & 0.000000 & -0.041311 & -0.059242 & 0.000001 & -0.098702\end{array}$

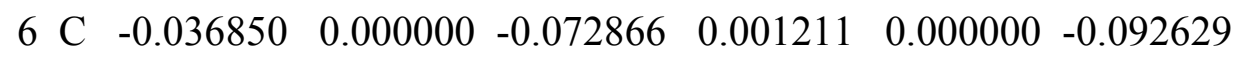

$\begin{array}{llllllll}7 \mathrm{H} & 0.052642 & 0.000000 & -0.074791 & 0.150369 & -0.000001 & 0.108559\end{array}$

$\begin{array}{llllllll}8 \mathrm{H} & 0.049865 & 0.000000 & 0.072465 & 0.142550 & -0.000001 & 0.107714\end{array}$

$\begin{array}{llllllll}9 & \mathrm{H} & 0.054694 & 0.000000 & 0.045304 & -0.153290 & 0.000001 & 0.115922\end{array}$

$\begin{array}{lllllll}10 \mathrm{H} & 0.054169 & 0.000000 & -0.092150 & -0.140843 & 0.000001 & 0.110077\end{array}$

$\begin{array}{llllllll}11 \mathrm{H} & 0.054296 & 0.000000 & -0.167630 & 0.009997 & 0.000000 & 0.110142\end{array}$ 
$\begin{array}{llllllll}12 & \mathrm{C} & 0.169028 & 0.000000 & -0.007057 & 0.030112 & -0.000006 & 0.193375\end{array}$

$\begin{array}{llllllll}13 & \mathrm{O} & -0.264111 & 0.000000 & -0.066341 & 0.083615 & 0.000001 & -0.315835\end{array}$

$\begin{array}{llllllll}14 & \mathrm{C} & -0.101848 & 0.000000 & 0.003227 & -0.001518 & 0.000001 & -0.243959\end{array}$

$\begin{array}{llllllll}15 & \mathrm{H} & 0.051110 & 0.000000 & -0.021652 & 0.087758 & -0.130206 & 0.104027\end{array}$

$\begin{array}{llllllll}16 & \mathrm{H} & 0.051111 & 0.000000 & -0.021657 & 0.087759 & 0.130205 & 0.104027\end{array}$

$\begin{array}{llllllll}17 & \mathrm{H} & 0.053528 & 0.000000 & 0.159316 & -0.030992 & 0.000003 & 0.109280\end{array}$

$\begin{array}{lllllll}\text { Tot }-0.000118 & 0.000000 & -0.197549 & 0.264196 & -0.000001 & -0.000118\end{array}$

\section{$\underline{2 b-F-g}$}

\begin{tabular}{|c|c|c|c|c|}
\hline \multirow{2}{*}{$\begin{array}{l}\text { Center } \\
\text { Number }\end{array}$} & Atomic & \multirow{2}{*}{$\begin{array}{l}\text { Atomic } \\
\text { Type }\end{array}$} & \multicolumn{2}{|c|}{ Coordinates (Angstroms) } \\
\hline & Number & & $X$ & Z \\
\hline 1 & 6 & 1.841227 & -1.383779 & 0.259351 \\
\hline 2 & 6 & 0.491647 & -1.051995 & 0.297927 \\
\hline 3 & 6 & 0.092629 & 0.266025 & 0.066285 \\
\hline 4 & 6 & 1.051626 & 1.244384 & -0.209080 \\
\hline 5 & 6 & 2.395355 & 0.907147 & -0.260427 \\
\hline 6 & 6 & 2.790647 & -0.408157 & -0.024138 \\
\hline 7 & 1 & 2.150190 & -2.405204 & 0.445286 \\
\hline 8 & 1 & -0.242076 & -1.821529 & 0.502372 \\
\hline 9 & 1 & 0.720153 & 2.262163 & -0.377325 \\
\hline 10 & 1 & 3.136902 & 1.665956 & -0.479441 \\
\hline 11 & 1 & 3.841439 & -0.671496 & -0.060484 \\
\hline 12 & 6 & -1.334237 & 0.693133 & 0.147735 \\
\hline 13 & 8 & -1.653792 & 1.856487 & 0.203089 \\
\hline 14 & 6 & -2.434902 & -0.360279 & 0.222109 \\
\hline 15 & 1 & -2.487784 & -0.765021 & 1.238026 \\
\hline
\end{tabular}




$$
\begin{array}{llllll}
16 & 9 & 0 & -2.208166 & -1.408207 & -0.650634 \\
17 & 1 & 0 & -3.378948 & 0.118225 & -0.036024
\end{array}
$$

Sum of electronic and zero-point Energies $=\quad-483.928007$

Summary of Natural Population Analysis:

Atom No Charge Core Valence Rydberg Total

$\begin{array}{lllllll}\mathrm{C} & 1 & -0.21160 & 1.99921 & 4.19500 & 0.01740 & 6.21160 \\ \mathrm{C} & 2 & -0.16691 & 1.99914 & 4.15248 & 0.01528 & 6.16691 \\ \mathrm{C} & 3 & -0.16980 & 1.99900 & 4.15273 & 0.01807 & 6.16980 \\ \mathrm{C} & 4 & -0.15456 & 1.99914 & 4.13808 & 0.01734 & 6.15456 \\ \mathrm{C} & 5 & -0.20918 & 1.99920 & 4.19313 & 0.01684 & 6.20918 \\ \mathrm{C} & 6 & -0.17787 & 1.99921 & 4.16175 & 0.01691 & 6.17787 \\ \mathrm{H} & 7 & 0.21479 & 0.00000 & 0.78373 & 0.00148 & 0.78521 \\ \mathrm{H} & 8 & 0.22287 & 0.00000 & 0.77522 & 0.00191 & 0.77713 \\ \mathrm{H} & 9 & 0.23350 & 0.00000 & 0.76457 & 0.00193 & 0.76650 \\ \mathrm{H} & 10 & 0.21496 & 0.00000 & 0.78360 & 0.00144 & 0.78504 \\ \mathrm{H} & 11 & 0.21294 & 0.00000 & 0.78569 & 0.00137 & 0.78706 \\ \mathrm{C} & 12 & 0.53594 & 1.99931 & 3.42511 & 0.03964 & 5.46406 \\ \mathrm{O} & 13 & -0.54117 & 1.99977 & 6.52670 & 0.01470 & 8.54117 \\ \mathrm{C} & 14 & 0.01074 & 1.99903 & 3.96751 & 0.02272 & 5.98926 \\ \mathrm{H} & 15 & 0.18002 & 0.00000 & 0.81810 & 0.00189 & 0.81998 \\ \mathrm{~F} & 16 & -0.38607 & 1.99994 & 7.37849 & 0.00763 & 9.38607 \\ \mathrm{H} & 17 & 0.19140 & 0.00000 & 0.80647 & 0.00213 & 0.80860\end{array}$

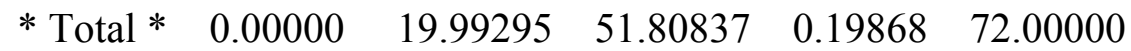


Hirshfeld charges, spin densities, dipoles, and CM5 charges using $\operatorname{IRadAn}=\quad 4$ :

Q-H S-H Dx Dy $\quad$ Dz Q-CM5

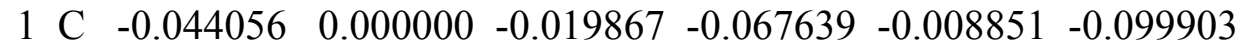

$\begin{array}{lllllll}2 \mathrm{C} & -0.036700 & 0.000000 & 0.032330 & -0.049337 & -0.008628 & -0.089871\end{array}$

$\begin{array}{llllllll}3 & \mathrm{C} & -0.024148 & 0.000000 & 0.055920 & 0.012003 & 0.001947 & -0.030288\end{array}$

$\begin{array}{llllllll}4 & \mathrm{C} & -0.029428 & 0.000000 & 0.013258 & 0.063469 & 0.011102 & -0.081192\end{array}$

$\begin{array}{llllllll}5 & \mathrm{C} & -0.042283 & 0.000000 & -0.051349 & 0.049051 & 0.015412 & -0.097912\end{array}$

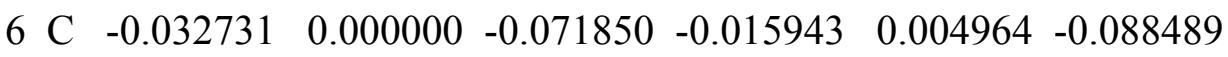

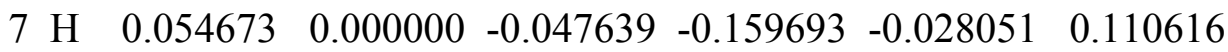

$\begin{array}{llllllll}8 \mathrm{H} & 0.049359 & 0.000000 & 0.083617 & -0.126385 & -0.039549 & 0.105003\end{array}$

$\begin{array}{llllllll}9 & \mathrm{H} & 0.056002 & 0.000000 & 0.021114 & 0.156792 & 0.032537 & 0.116859\end{array}$

$\begin{array}{llllllll}10 & \mathrm{H} & 0.055188 & 0.000000 & -0.114912 & 0.119037 & 0.034136 & 0.111118\end{array}$

$\begin{array}{llllllll}11 & \mathrm{H} & 0.055737 & 0.000000 & -0.163309 & -0.040768 & 0.005897 & 0.111578\end{array}$

$\begin{array}{llllllll}12 & \mathrm{C} & 0.165162 & 0.000000 & 0.005392 & -0.027322 & 0.008119 & 0.191709\end{array}$

$\begin{array}{llllllll}13 & \mathrm{O} & -0.253228 & 0.000000 & -0.045344 & -0.087421 & 0.011523 & -0.304342\end{array}$

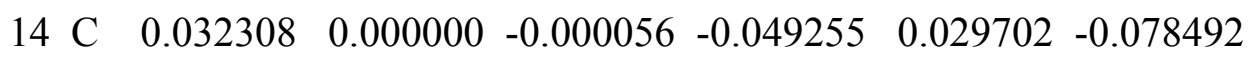

$\begin{array}{llllllll}15 & \mathrm{H} & 0.059199 & 0.000000 & 0.019891 & -0.056325 & -0.151863 & 0.107251\end{array}$

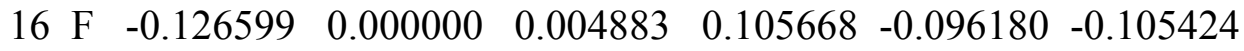

$\begin{array}{llllllll}17 & \mathrm{H} & 0.061533 & 0.000000 & 0.149977 & 0.059633 & 0.037598 & 0.121770\end{array}$

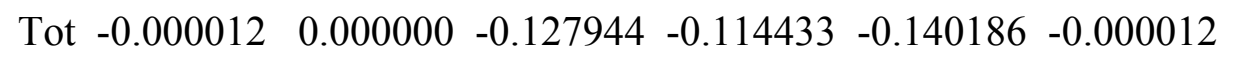

\section{$\underline{\text { 2b-F-s }}$}

\begin{tabular}{|c|c|c|c|c|}
\hline Center & Atomic & Atomic & Coordinate & (Angstroms) \\
\hline Number & Number & Type & $\mathrm{X}$ & Z \\
\hline 1 & 6 & -2.136190 & -1.368630 & 0.000123 \\
\hline 2 & 6 & -0.760296 & -1.167701 & 0.000333 \\
\hline
\end{tabular}




$\begin{array}{llllll}3 & 6 & 0 & -0.241171 & 0.128662 & 0.000211 \\ 4 & 6 & 0 & -1.111079 & 1.220705 & -0.000037 \\ 5 & 6 & 0 & -2.483330 & 1.018806 & -0.000201 \\ 6 & 6 & 0 & -2.996936 & -0.276323 & -0.000157 \\ 7 & 1 & 0 & -2.534600 & -2.375846 & 0.000175 \\ 8 & 1 & 0 & -0.103596 & -2.030384 & 0.000628 \\ 9 & 1 & 0 & -0.688245 & 2.218305 & -0.000138 \\ 10 & 1 & 0 & -3.155145 & 1.868633 & -0.000403 \\ 11 & 1 & 0 & -4.069021 & -0.434103 & -0.000345 \\ 12 & 6 & 0 & 1.231262 & 0.403631 & 0.000085 \\ 13 & 8 & 0 & 1.674490 & 1.521275 & 0.000276 \\ 14 & 6 & 0 & 2.163156 & -0.801448 & -0.000428 \\ 15 & 1 & 0 & 1.982024 & -1.411653 & 0.890065 \\ 16 & 9 & 0 & 3.466428 & -0.393421 & -0.000005 \\ 17 & 1 & 0 & 1.982320 & -1.410590 & -0.891715\end{array}$

Sum of electronic and zero-point Energies $=\quad-483.926949$ Summary of Natural Population Analysis:

\begin{tabular}{ccccccc} 
Atom No & Charge & Core & Valence & Rydberg & Total \\
\hline------------------------------------------------------- \\
C & 1 & -0.20829 & 1.99921 & 4.19146 & 0.01762 & 6.20829 \\
C & 2 & -0.18516 & 1.99914 & 4.17105 & 0.01497 & 6.18516 \\
C & 3 & -0.16525 & 1.99900 & 4.14930 & 0.01695 & 6.16525 \\
C & 4 & -0.15627 & 1.99914 & 4.13941 & 0.01772 & 6.15627 \\
C & 5 & -0.20405 & 1.99920 & 4.18807 & 0.01678 & 6.20405 \\
C & 6 & -0.18079 & 1.99921 & 4.16467 & 0.01691 & 6.18079 \\
H & 7 & 0.21508 & 0.00000 & 0.78343 & 0.00149 & 0.78492
\end{tabular}




$\begin{array}{ccccccc}\mathrm{H} & 8 & 0.20946 & 0.00000 & 0.78882 & 0.00173 & 0.79054 \\ \mathrm{H} & 9 & 0.23702 & 0.00000 & 0.76097 & 0.00201 & 0.76298 \\ \mathrm{H} & 10 & 0.21597 & 0.00000 & 0.78261 & 0.00142 & 0.78403 \\ \mathrm{H} & 11 & 0.21417 & 0.00000 & 0.78447 & 0.00136 & 0.78583 \\ \mathrm{C} & 12 & 0.53042 & 1.99932 & 3.43040 & 0.03986 & 5.46958 \\ \mathrm{O} & 13 & -0.52415 & 1.99976 & 6.50920 & 0.01518 & 8.52415 \\ \mathrm{C} & 14 & 0.00864 & 1.99904 & 3.96940 & 0.02292 & 5.99136 \\ \mathrm{H} & 15 & 0.18052 & 0.00000 & 0.81753 & 0.00195 & 0.81948 \\ \mathrm{~F} & 16 & -0.36788 & 1.99994 & 7.35897 & 0.00897 & 9.36788 \\ \mathrm{H} & 17 & 0.18055 & 0.00000 & 0.81751 & 0.00195 & 0.81945\end{array}$

* Total * $\quad 0.00000 \quad 19.99298 \quad 51.80726 \quad 0.19977 \quad 72.00000$

Hirshfeld charges, spin densities, dipoles, and CM5 charges using IRadAn $=\quad 4$ :

Q-H S-H Dx $\quad$ Dy $\quad$ Dz $\quad$ Q-CM5

$\begin{array}{lllllll}1 \mathrm{C} & -0.044245 & 0.000000 & 0.028446 & -0.065422 & -0.000007 & -0.100159\end{array}$

$\begin{array}{lllllll}2 \mathrm{C} & -0.042127 & 0.000000 & -0.037649 & -0.053059 & -0.000023 & -0.095490\end{array}$

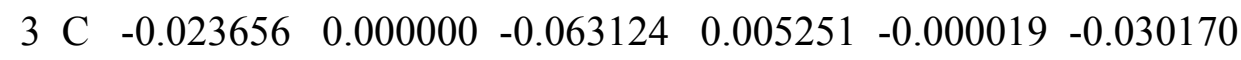

$\begin{array}{llllllll}4 & \mathrm{C} & -0.028193 & 0.000000 & -0.018884 & 0.065374 & -0.000005 & -0.079969\end{array}$

$\begin{array}{llllllll}5 & \mathrm{C} & -0.039509 & 0.000000 & 0.047076 & 0.057144 & 0.000005 & -0.095132\end{array}$

$\begin{array}{llllllll}6 & \mathrm{C} & -0.033025 & 0.000000 & 0.074247 & -0.007684 & 0.000012 & -0.088817\end{array}$

$\begin{array}{llllllll}7 \mathrm{H} & 0.054941 & 0.000000 & 0.062924 & -0.157269 & -0.000008 & 0.110884\end{array}$

$\begin{array}{llllllll}8 \mathrm{H} & 0.051160 & 0.000000 & -0.084754 & -0.137895 & -0.000050 & 0.108928\end{array}$

$\begin{array}{llllllll}9 \mathrm{H} & 0.057780 & 0.000000 & -0.034125 & 0.158555 & 0.000021 & 0.118778\end{array}$

$\begin{array}{llllllll}10 & \mathrm{H} & 0.056495 & 0.000000 & 0.104297 & 0.133841 & 0.000031 & 0.112441\end{array}$

$\begin{array}{llllllll}11 & \mathrm{H} & 0.056558 & 0.000000 & 0.167486 & -0.024029 & 0.000030 & 0.112428\end{array}$

12 C $\quad 0.1619120 .000000 \quad 0.010260-0.032769-0.0000010 .189074$

$\begin{array}{llllllll}13 & \mathrm{O} & -0.245318 & 0.000000 & 0.055864 & -0.069101 & 0.000018 & -0.292872\end{array}$

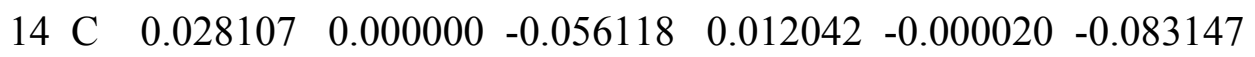




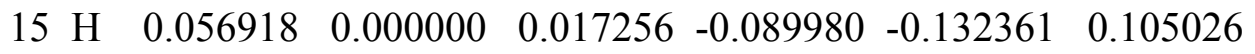

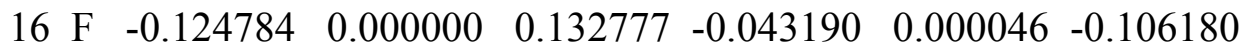

$\begin{array}{lllllll}17 \mathrm{H} & 0.056931 & 0.000000 & 0.017215 & -0.089822 & 0.132487 & 0.114320\end{array}$

$\begin{array}{lllllll}\text { Tot }-0.000056 & 0.000000 & 0.423195 & -0.338015 & 0.000155 & -0.000056\end{array}$

\section{$\underline{2 b-F 2-s g}$}

\begin{tabular}{|c|c|c|c|c|}
\hline \multirow{2}{*}{$\begin{array}{l}\text { Center } \\
\text { Number }\end{array}$} & Atomic & Atomic & \multicolumn{2}{|c|}{ Coordinates (Angstroms) } \\
\hline & Number & Type & $\mathrm{X}$ & Z \\
\hline 1 & 6 & 2.225316 & -1.391481 & -0.185986 \\
\hline 2 & 6 & 0.871467 & -1.075864 & -0.194131 \\
\hline 3 & 6 & 0.465583 & 0.252059 & -0.041569 \\
\hline 4 & 6 & 1.422976 & 1.257525 & 0.121212 \\
\hline 5 & 6 & 2.771673 & 0.938165 & 0.132812 \\
\hline 6 & 6 & 3.173138 & -0.387263 & -0.022262 \\
\hline 7 & 1 & 2.539140 & -2.421435 & -0.302501 \\
\hline 8 & 1 & 0.144209 & -1.870905 & -0.303519 \\
\hline 9 & 1 & 1.084980 & 2.280438 & 0.236293 \\
\hline 10 & 1 & 3.512209 & 1.718250 & 0.261327 \\
\hline 11 & 1 & 4.227833 & -0.636733 & -0.014224 \\
\hline 12 & 6 & -0.966876 & 0.662330 & -0.067644 \\
\hline 13 & 8 & -1.328840 & 1.801931 & 0.041776 \\
\hline 14 & 6 & -2.022247 & -0.442540 & -0.284254 \\
\hline 15 & 9 & -3.253572 & 0.086617 & -0.251677 \\
\hline 16 & 9 & -1.928012 & -1.352492 & $2 \quad 0.726408$ \\
\hline 17 & 1 & -1.889584 & -0.969772 & $2-1.233231$ \\
\hline
\end{tabular}


Sum of electronic and zero-point Energies $=$ $-583.180416$

Summary of Natural Population Analysis:

\begin{tabular}{llllllll} 
Atom & No & Charge & Core & Valence & Rydberg & Total \\
\hline C & 1 & -0.20906 & 1.99921 & 4.19236 & 0.01749 & 6.20906 \\
C & 2 & -0.17229 & 1.99914 & 4.15789 & 0.01526 & 6.17229 \\
C & 3 & -0.17577 & 1.99901 & 4.15930 & 0.01747 & 6.17577 \\
C & 4 & -0.15209 & 1.99914 & 4.13535 & 0.01760 & 6.15209 \\
C & 5 & -0.20598 & 1.99920 & 4.18999 & 0.01679 & 6.20598 \\
C & 6 & -0.17384 & 1.99921 & 4.15779 & 0.01685 & 6.17384 \\
H & 7 & 0.21670 & 0.00000 & 0.78182 & 0.00148 & 0.78330 \\
H & 8 & 0.21943 & 0.00000 & 0.77857 & 0.00200 & 0.78057 \\
H & 9 & 0.23589 & 0.00000 & 0.76210 & 0.00201 & 0.76411 \\
H & 10 & 0.21705 & 0.00000 & 0.78154 & 0.00141 & 0.78295 \\
H & 11 & 0.21485 & 0.00000 & 0.78380 & 0.00135 & 0.78515 \\
C & 12 & 0.50847 & 1.99929 & 3.45147 & 0.04076 & 5.49153 \\
O & 13 & -0.50481 & 1.99977 & 6.49021 & 0.01484 & 8.50481 \\
C & 14 & 0.56402 & 1.99919 & 3.39693 & 0.03986 & 5.43598 \\
F & 15 & -0.35283 & 1.99993 & 7.34465 & 0.00825 & 9.35283 \\
F & 16 & -0.37328 & 1.99994 & 7.36682 & 0.00652 & 9.37328 \\
H & 17 & 0.14356 & 0.00000 & 0.85409 & 0.00234 & 0.85644 \\
\hline
\end{tabular}

$\begin{array}{llllll}* \text { Total * } & 0.00000 & 21.99302 & 57.78468 & 0.22230 & 80.00000\end{array}$

Hirshfeld charges, spin densities, dipoles, and CM5 charges using $\operatorname{IRadAn}=\quad 4$ :
Q-H S-H Dx $\quad$ Dy $\quad$ Dz $\quad$ Q-CM5
$\begin{array}{llllllll}1 & C & -0.041658 & 0.000000 & 0.021850 & -0.069273 & -0.003432 & -0.097570\end{array}$ 


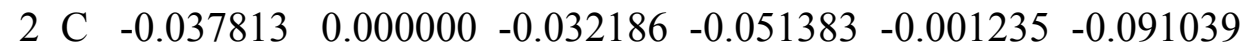
$\begin{array}{llllllll}3 & \mathrm{C} & -0.025334 & 0.000000 & -0.054597 & 0.013084 & 0.005145 & -0.031475\end{array}$
$\begin{array}{llllllll}4 & C & -0.026463 & 0.000000 & -0.014510 & 0.065833 & 0.010959 & -0.078244\end{array}$
$\begin{array}{llllllll}5 & \mathrm{C} & -0.038880 & 0.000000 & 0.052988 & 0.051633 & 0.010692 & -0.094520\end{array}$
$\begin{array}{llllllll}6 & C & -0.029167 & 0.000000 & 0.074042 & -0.014894 & 0.002957 & -0.084936\end{array}$
$\begin{array}{llllllll}7 & \mathrm{H} & 0.056603 & 0.000000 & 0.049272 & -0.162065 & -0.017273 & 0.112576\end{array}$
$\begin{array}{llllllll}8 \mathrm{H} & 0.049873 & 0.000000 & -0.083023 & -0.132092 & -0.023109 & 0.105832\end{array}$
$\begin{array}{llllllll}9 \mathrm{H} & 0.058395 & 0.000000 & -0.021638 & 0.160161 & 0.020273 & 0.119362\end{array}$
$\begin{array}{llllllll}10 \mathrm{H} & 0.057420 & 0.000000 & 0.115655 & 0.123188 & 0.020639 & 0.113367\end{array}$
$\begin{array}{llllllll}11 & \mathrm{H} & 0.057848 & 0.000000 & 0.165122 & -0.038758 & 0.001659 & 0.113702\end{array}$
$\begin{array}{llllllll}12 & \mathrm{C} & 0.159242 & 0.000000 & 0.001798 & -0.027409 & -0.006399 & 0.188848\end{array}$
$\begin{array}{llllllll}13 & \mathrm{O} & -0.231551 & 0.000000 & 0.048405 & -0.062135 & -0.002213 & -0.278106\end{array}$
$\begin{array}{llllllll}14 & \mathrm{C} & 0.140915 & 0.000000 & -0.041995 & -0.016668 & 0.021916 & 0.058011\end{array}$
$\begin{array}{llllllll}15 & F & -0.101168 & 0.000000 & 0.103778 & -0.043454 & -0.008409 & -0.086361\end{array}$

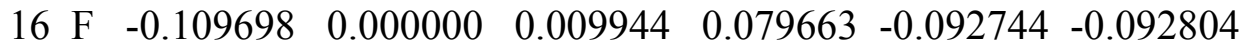
$\begin{array}{llllllll}17 & \mathrm{H} & 0.061289 & 0.000000 & 0.008198 & -0.076838 & -0.147620 & 0.123212\end{array}$
$\begin{array}{lllllll}\text { Tot } & -0.000147 & 0.000000 & 0.403105 & -0.201407 & -0.208194 & -0.000147\end{array}$

\section{$\underline{\text { 2b-F2-gg }}$}

\begin{tabular}{cccccc} 
Center & \multicolumn{2}{c}{ Atomic } & \multicolumn{2}{c}{ Atomic } & \multicolumn{2}{c}{ Coordinates (Angstroms) } \\
Number & Number & Type & $X$ & $Y$ & $Z$ \\
- & & & & & \\
- & & & & & \\
1 & 6 & 0 & -1.951746 & -1.487634 & -0.000013 \\
2 & 6 & 0 & -0.635378 & -1.041493 & -0.000002 \\
3 & 6 & 0 & -0.370375 & 0.330691 & 0.000009 \\
4 & 6 & 0 & -1.426339 & 1.246832 & 0.000002 \\
5 & 6 & 0 & -2.736612 & 0.796212 & -0.000008 \\
6 & 6 & 0 & -2.999034 & -0.572775 & -0.000014
\end{tabular}




$\begin{array}{llllll}7 & 1 & 0 & -2.158675 & -2.550733 & -0.000021 \\ 8 & 1 & 0 & 0.173748 & -1.760343 & -0.000002 \\ 9 & 1 & 0 & -1.196171 & 2.305572 & 0.000006 \\ 10 & 1 & 0 & -3.554621 & 1.506179 & -0.000011 \\ 11 & 1 & 0 & -4.023641 & -0.926085 & -0.000023 \\ 12 & 6 & 0 & 1.010954 & 0.880589 & 0.000015 \\ 13 & 8 & 0 & 1.258351 & 2.060456 & -0.000108 \\ 14 & 6 & 0 & 2.208518 & -0.083077 & -0.000045 \\ 15 & 9 & 0 & 2.163623 & -0.886314 & -1.094107 \\ 16 & 1 & 0 & 3.143922 & 0.476333 & -0.000292 \\ 17 & 9 & 0 & 2.164010 & -0.885979 & 1.094279\end{array}$

Sum of electronic and zero-point Energies=

$-583.183563$

Summary of Natural Population Analysis:

\begin{tabular}{|c|c|c|c|c|c|c|}
\hline Aton & $\mathrm{n}$ & Charge & Core & Valence & Rydberg & Total \\
\hline $\mathrm{C}$ & 1 & -0.21071 & 1.99921 & 4.19423 & 0.01727 & 6.21071 \\
\hline $\mathrm{C}$ & 2 & -0.16027 & 1.99914 & 4.14568 & 0.01545 & 6.16027 \\
\hline $\mathrm{C}$ & 3 & -0.17924 & 1.99900 & 4.16240 & 0.01784 & 6.17924 \\
\hline $\mathrm{C}$ & 4 & -0.15132 & 1.99914 & 4.13482 & 0.01737 & 6.15132 \\
\hline $\mathrm{C}$ & 5 & -0.20926 & 1.99920 & 4.19328 & 0.01677 & 6.20926 \\
\hline $\mathrm{C}$ & 6 & -0.17179 & 1.99921 & 4.15576 & 0.01681 & 6.17179 \\
\hline $\mathrm{H}$ & 7 & 0.21659 & 0.00000 & 0.78194 & 0.00147 & 0.78341 \\
\hline $\mathrm{H}$ & 8 & 0.22880 & 0.00000 & 0.76891 & 0.00229 & 0.77120 \\
\hline $\mathrm{H}$ & 9 & 0.23418 & 0.00000 & 0.76388 & 0.00194 & 0.76582 \\
\hline $\mathrm{H}$ & 10 & 0.21617 & 0.00000 & 0.78240 & 0.00143 & 0.78383 \\
\hline $\mathrm{H}$ & 11 & 0.21388 & 0.00000 & 0.78476 & 0.00136 & 0.78612 \\
\hline
\end{tabular}




$\begin{array}{lllllll}\text { C } & 12 & 0.51049 & 1.99928 & 3.44949 & 0.04074 & 5.48951 \\ \text { O } & 13 & -0.52309 & 1.99977 & 6.50851 & 0.01481 & 8.52309 \\ \text { C } & 14 & 0.56921 & 1.99916 & 3.39090 & 0.04073 & 5.43079 \\ \text { F } & 15 & -0.36949 & 1.99993 & 7.36344 & 0.00612 & 9.36949 \\ \text { H } & 16 & 0.15533 & 0.00000 & 0.84195 & 0.00272 & 0.84467 \\ \text { F } & 17 & -0.36949 & 1.99993 & 7.36344 & 0.00612 & 9.36949\end{array}$

$\begin{array}{llllll}* \text { Total * } & 0.00000 & 21.99298 & 57.78579 & 0.22122 & 80.00000\end{array}$

Hirshfeld charges, spin densities, dipoles, and CM5 charges using IRadAn $=\quad 4$ :

Q-H S-H Dx Dy $\quad$ Dz $\quad$ Q-CM5

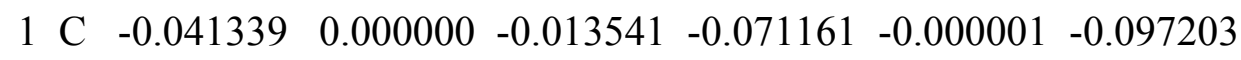

$\begin{array}{llllllll}2 & \mathrm{C} & -0.033315 & 0.000000 & 0.032055 & -0.048482 & -0.000002 & -0.086438\end{array}$

$\begin{array}{llllllll}3 & \mathrm{C} & -0.025571 & 0.000000 & 0.049146 & 0.018424 & -0.000001 & -0.031542\end{array}$

$\begin{array}{llllllll}4 \mathrm{C} & -0.027560 & 0.000000 & 0.007770 & 0.066320 & -0.000001 & -0.079329\end{array}$

$\begin{array}{llllllll}5 & \mathrm{C} & -0.040884 & 0.000000 & -0.057634 & 0.045672 & 0.000000 & -0.096548\end{array}$

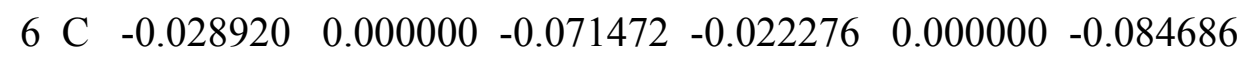

$\begin{array}{llllllll}7 \mathrm{H} & 0.056560 & 0.000000 & -0.031558 & -0.167127 & -0.000001 & 0.112531\end{array}$

$\begin{array}{llllllll}8 & \mathrm{H} & 0.049589 & 0.000000 & 0.086805 & -0.125892 & 0.000006 & 0.104202\end{array}$

$\begin{array}{llllllll}9 \mathrm{H} & 0.056988 & 0.000000 & 0.004285 & 0.161852 & 0.000001 & 0.117974\end{array}$

$\begin{array}{llllllll}10 & \mathrm{H} & 0.056390 & 0.000000 & -0.127625 & 0.111865 & 0.000000 & 0.112346\end{array}$

$\begin{array}{llllllll}11 & \mathrm{H} & 0.057244 & 0.000000 & -0.159830 & -0.055087 & -0.000001 & 0.113102\end{array}$

$\begin{array}{llllllll}12 & \mathrm{C} & 0.160670 & 0.000000 & 0.007888 & -0.017665 & 0.000033 & 0.189592\end{array}$

$\begin{array}{llllllll}13 & \mathrm{O} & -0.239015 & 0.000000 & -0.041243 & -0.078690 & 0.000001 & -0.288984\end{array}$

$\begin{array}{llllllll}14 & \mathrm{C} & 0.145563 & 0.000000 & 0.012585 & -0.061548 & 0.000001 & 0.063032\end{array}$

$\begin{array}{llllllll}15 & F & -0.106844 & 0.000000 & -0.005068 & 0.074127 & 0.094514 & -0.090010\end{array}$

$\begin{array}{llllllll}16 & \mathrm{H} & 0.067295 & 0.000000 & 0.153782 & 0.074024 & -0.000040 & 0.122592\end{array}$

$\begin{array}{llllllll}17 & \text { F } & -0.106858 & 0.000000 & -0.005075 & 0.074112 & -0.094534 & -0.080640\end{array}$

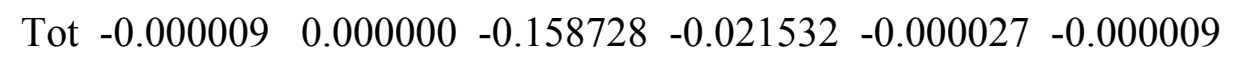




\section{2b-F3-sgg}

\begin{tabular}{|c|c|c|c|c|}
\hline \multirow{2}{*}{$\begin{array}{l}\text { Center } \\
\text { Number }\end{array}$} & Atomic & Atomic & \multicolumn{2}{|c|}{ Coordinates (Angstroms } \\
\hline & Number & Type & $X$ & $\mathrm{Z}$ \\
\hline 1 & 6 & -2.326021 & -1.430867 & -0.000196 \\
\hline 2 & 6 & -0.990467 & -1.047057 & -0.000242 \\
\hline 3 & 6 & -0.661351 & 0.311378 & 0.000010 \\
\hline 4 & 6 & -1.674218 & 1.275552 & 0.000305 \\
\hline 5 & 6 & -3.003777 & 0.886484 & 0.000360 \\
\hline 6 & 6 & -3.329631 & -0.468556 & 0.000109 \\
\hline 7 & 1 & -2.581490 & -2.483386 & -0.000396 \\
\hline 8 & 1 & -0.220401 & -1.807560 & -0.000491 \\
\hline 9 & 1 & -1.396003 & 2.322675 & 0.000493 \\
\hline 10 & 1 & -3.787383 & 1.634165 & 0.000603 \\
\hline 11 & 1 & -4.369493 & -0.774255 & 0.000154 \\
\hline 12 & 6 & 0.738697 & 0.810258 & -0.000014 \\
\hline 13 & 8 & 1.041707 & 1.970546 & 0.000067 \\
\hline 14 & 6 & 1.885924 & -0.235123 & -0.000078 \\
\hline 15 & 9 & 1.808962 & -1.023467 & -1.082466 \\
\hline 16 & 9 & 1.808816 & -1.023705 & 1.082135 \\
\hline 17 & 9 & 3.069575 & 0.350680 & 0.000063 \\
\hline
\end{tabular}

Sum of electronic and zero-point Energies=

$-682.445863$

Summary of Natural Population Analysis:

Atom No Charge Core Valence Rydberg Total 


$\begin{array}{lllllll}\text { C } & 1 & -0.20927 & 1.99921 & 4.19265 & 0.01742 & 6.20927 \\ \text { C } & 2 & -0.16159 & 1.99914 & 4.14738 & 0.01507 & 6.16159 \\ \text { C } & 3 & -0.18353 & 1.99901 & 4.16715 & 0.01737 & 6.18353 \\ \text { C } & 4 & -0.14905 & 1.99914 & 4.13231 & 0.01760 & 6.14905 \\ \text { C } & 5 & -0.20729 & 1.99920 & 4.19128 & 0.01681 & 6.20729 \\ \text { C } & 6 & -0.16848 & 1.99921 & 4.15249 & 0.01678 & 6.16848 \\ \text { H } & 7 & 0.21819 & 0.00000 & 0.78035 & 0.00146 & 0.78181 \\ \text { H } & 8 & 0.22646 & 0.00000 & 0.77147 & 0.00207 & 0.77354 \\ \text { H } & 9 & 0.23553 & 0.00000 & 0.76244 & 0.00203 & 0.76447 \\ \text { H } & 10 & 0.21794 & 0.00000 & 0.78065 & 0.00141 & 0.78206 \\ \text { H } & 11 & 0.21551 & 0.00000 & 0.78314 & 0.00135 & 0.78449 \\ \text { C } & 12 & 0.49550 & 1.99924 & 3.46329 & 0.04196 & 5.50450 \\ \text { O } & 13 & -0.49601 & 1.99977 & 6.48117 & 0.01508 & 8.49601 \\ \text { C } & 14 & 1.01948 & 1.99944 & 2.92124 & 0.05985 & 4.98052 \\ \text { F } & 15 & -0.35805 & 1.99992 & 7.35106 & 0.00707 & 9.35805 \\ \text { F } & 16 & -0.35805 & 1.99992 & 7.35106 & 0.00707 & 9.35805 \\ \text { F } & 17 & -0.33730 & 1.99991 & 7.32871 & 0.00867 & 9.33730\end{array}$

$\begin{array}{llllll}* \text { Total * } & 0.00000 & 23.99311 & 63.75782 & 0.24907 & 88.00000\end{array}$

Hirshfeld charges, spin densities, dipoles, and CM5 charges using $\operatorname{IRadAn}=\quad 4$ :

Q-H S-H Dx Dy $\quad$ Dz $\quad$ Q-CM5

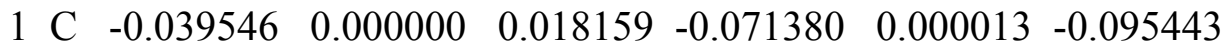

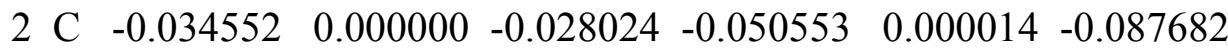

$\begin{array}{llllllll}3 & \mathrm{C} & -0.026541 & 0.000000 & -0.048814 & 0.017584 & 0.000003 & -0.032428\end{array}$

$\begin{array}{llllllll}4 & C & -0.025238 & 0.000000 & -0.010888 & 0.067364 & -0.000011 & -0.077007\end{array}$

$\begin{array}{llllllll}5 & \mathrm{C} & -0.038403 & 0.000000 & 0.056830 & 0.049452 & -0.000016 & -0.094079\end{array}$ 


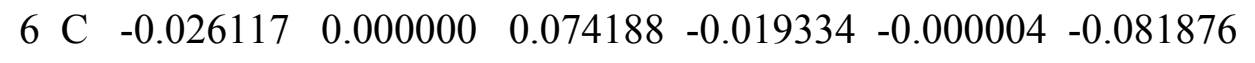
$\begin{array}{llllllll}7 & \mathrm{H} & 0.058091 & 0.000000 & 0.039760 & -0.166335 & 0.000032 & 0.114065\end{array}$
$\begin{array}{llllllll}8 \mathrm{H} & 0.049108 & 0.000000 & -0.080124 & -0.132131 & 0.000039 & 0.103647\end{array}$
$\begin{array}{llllllll}9 \mathrm{H} & 0.058943 & 0.000000 & -0.012164 & 0.163063 & -0.000034 & 0.119995\end{array}$
$\begin{array}{llllllll}10 & \mathrm{H} & 0.058200 & 0.000000 & 0.122981 & 0.118442 & -0.000038 & 0.114169\end{array}$
$\begin{array}{llllllll}11 & \mathrm{H} & 0.059000 & 0.000000 & 0.163183 & -0.047907 & -0.000007 & 0.114852\end{array}$
$\begin{array}{llllllll}12 & \mathrm{C} & 0.157685 & 0.000000 & -0.000308 & -0.020116 & 0.000011 & 0.189087\end{array}$
$\begin{array}{llllllll}13 & \mathrm{O} & -0.224012 & 0.000000 & 0.044924 & -0.055689 & -0.000001 & -0.269838\end{array}$
$\begin{array}{llllllll}14 & \mathrm{C} & 0.238059 & 0.000000 & -0.037054 & -0.038032 & 0.000005 & 0.182008\end{array}$
$\begin{array}{llllllll}15 & \text { F } & -0.091682 & 0.000000 & 0.006894 & 0.059322 & -0.078328 & -0.069393\end{array}$
$\begin{array}{llllllll}16 & \mathrm{~F} & -0.091687 & 0.000000 & 0.006882 & 0.059342 & 0.078318 & -0.069398\end{array}$
$\begin{array}{llllllll}17 & \text { F } & -0.081363 & 0.000000 & 0.080222 & -0.036784 & 0.000009 & -0.060734\end{array}$
$\begin{array}{lllllll}\text { Tot } & -0.000056 & 0.000000 & 0.396645 & -0.103693 & 0.000005 & -0.000056\end{array}$

\section{References}

1. Besseau, F.; Luçon, M.; Laurence, C.; Berthelot, M.” Hydrogen-bond basicity pKHB scale of aldehydes and ketones" J. Chem. Soc., Perkin Trans. 2, 1998, 101-107. 